\title{
Microwave Transitions of Cold Rydberg Atom Pairs
}

\author{
Jeonghun Lee \\ Daegu, Republic of Korea
}

B.S., Illinois Institute of Technology, 2011

\begin{abstract}
A Dissertation presented to the Graduate Faculty of the University of Virginia in Candidacy for the Degree of

Doctor of Philosophy
\end{abstract}

Department of Physics

University of Virginia

August, 2017 


\begin{abstract}
This dissertation consists of three microwave spectroscopy experiments performed with cold Rb Rydberg atoms in Magneto Optical Trap.

The first experiment is a systematic study of a particular type of transition which was first observed in 2012 that involves a pair of Rydberg atoms. In this transition, even though only one microwave photon is absorbed, both atoms in the pair change states. In particular, we describe the transition with the configuration interaction (CI) model and experimentally verify the scaling of fractional population transfer predicted by the CI model.

The second experiment builds on the first experiment and reports observations of additional single and multi photon microwave transitions that are allowed due to the same CI as in the first experiment. The observations show clearly that this type of transition involving a pair of Rydberg atoms are a lot more common than initially thought. We show that the transitions can be conveniently described as Forster resonances of Floquet states.

The last experiment reports on the technique we used to measure the $n g$ and $n h$ series quantum defects and the ionic dipole and quadrupole polarizabilities of ${ }^{85} \mathrm{Rb}$. The technique allows us to obtain zero field intervals of $(n+1) d_{5 / 2} \rightarrow n g$ and $(n+1) d_{5 / 2} \rightarrow n h$ intervals despite the fact that we can only control the stray electric field in one direction. Using the technique, the quantum defects of $n g$ and $n h$ were determined to be $0.0039741(16)$ and $0.0014066(57)$, respectively. The ionic
\end{abstract}


dipole and quadrupole polarizabilities were determined to be $9.12(2) a_{0}^{3}$ and $14(3) a_{0}^{5}$, respectively. 


\section{Acknowledgement}

As I approach the end of my career as a graduate student, I realize how fortunate I have been in every corner of my life. I have come across many great people to whom I am much indebted for every little thing I managed to achieve. Although it is not possible to list all of their names here, I would like to express my gratitude to each one of them for one reason or another.

With that being said, there are people to whom I would like to express special thanks.

First and foremost, I cannot express how grateful I am to my parents for everything they have done for me. They always have been extremely encouraging and supportive. Without their support, I would have never finished.

The Fay family in New Hampshire took care of me when I first came to America as an exchange student 14 years ago. During the 5 months I spent with them, I was treated as a part of the family and learned many things that later proved to be immensely helpful for living in America. I am fortunate to have been welcomed to this country by such wonderful people.

Dr. Atsushi Yoshida, who taught me physics in high school for two years, first 
got me seriously interested in physics. Before taking physics classes with him, I never considered science as my career. He is an outstanding teacher, and I am fortunate to have been his student. I am glad that he is still around and we still hang out.

I also would like to thank all my colleagues over the years: Dr. Hyunwook Park, Dr. Jirakan Nunkaew, Dr. Vincent Carrat, Dr. Alexandr Arakelyan, Eric Magnuson, Safra Niyaz, Kapila Wijayaratne, Javed Iqbal, and Phatthamon Kongkhambut. It was both productive and pleasant to have worked with them. I spent some great time with them both in and out of the lab. Especially memorable were the trips we made together when attending conferences. In particular, I would like to thank Dr. Jirakan Nunkaew, Javed Iqbal, and Phatthamon Kongkhambut for working directly with me on experiments. We started out as colleagues, but became good friend after sharing so much time together in the lab.

Finally, I want to thank my advisor, Dr. Gallagher, for everything he taught me during the last 5 years. I have great respect for him both as a scientist and a person, and it was an honor and a great pleasure working for him. I am definitely going to miss those times he invited our group to his house for dinner. Graduate school was certainly not easy. Luckily, I was blessed with a great advisor. 


\section{Contents}

Acknowledgement $\quad$ V

1 Introduction 1

1.1 Rydberg Atoms . . . . . . . . . . . . . . . . . . . 1

1.2 Thesis Outline . . . . . . . . . . . . . . . . . . . 4

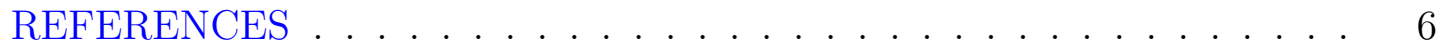

2 Experimental Approach $\quad 8$

2.1 Magneto Optical Trap (MOT) . . . . . . . . . . . . . . . . . 9

2.1.1 Principle of Operation . . . . . . . . . . . . . . 10

2.1.2 Vacuum Chamber . . . . . . . . . . . . . . . . . . . 11

2.1.3 Electric Field . . . . . . . . . . . . . . . . . . . . . . . 13

2.1.4 External Cavity Diode Laser (ECDL) . . . . . . . . . . . 15

2.1.5 Magnetic Field . . . . . . . . . . . . . . . . . 18

2.1.6 Determining the Trap Parameters . . . . . . . . . . . . . . 19

2.2 Excitation of Rydberg Atoms _. . . . . . . . . . . . . 24

2.2 .1 Dye Amplified Diode Laser . . . . . . . . . . . . . . . . . . . 24 
2.2 .2 Dye Laser . . . . . . . . . . . . . . . . . . . . . . . 25

2.3 Microwave Setup . . . . . . . . . . . . . . . . . . . 26

2.4 Detection and Data Acquisition . . . . . . . . . . . . . . 28

2.4 .1 Field Ionization . . . . . . . . . . . . . . . . . . 28

2.4.2 Ion Detection and Electron Detection . . . . . . . . . . . . 30

2.5 The Timing Diagram . . . . . . . . . . . . . . . . . . 33

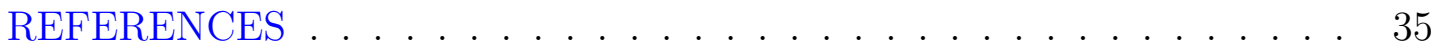

3 Molecular Transition $\quad 36$

3.1 Introduction . . . . . . . . . . . . . . . 36

3.2 Theory - Configuration Interaction Model _ . . . . . . . . . . . 38

3.3 Experimental Procedure . . . . . . . . . . . . . . . . . . . . . 45

3.4 Results and Analysis . . . . . . . . . . . . . . . . . 47

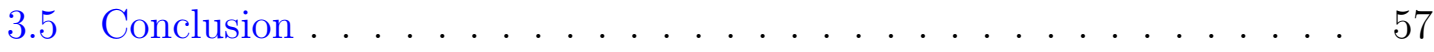

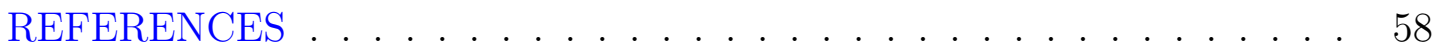

4 Multiphoton Molecular Transitions $\quad 60$

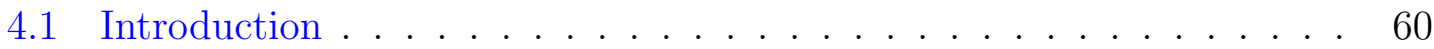

4.2 Floquet Model of Forster Energy Transfer . . . . . . . . . . . . . 62

4.3 Experimental Approach _. . . . . . . . . . . . . . . . . . 74

4.4 Observations . . . . . . . . . . . . . . . . . 77

4.5 Conclusion . . . . . . . . . . . . . . . . . . . . . 91

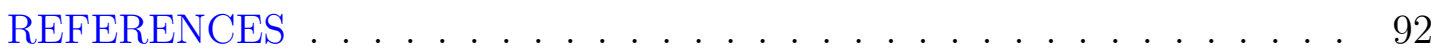


5 Quantum Defects and Ionic Dipole and Quadrupole Polarizabilities 93

5.1 Introduction . . . . . . . . . . . . . . . . . 93

5.2 Principle . . . . . . . . . . . . . . . . . . . . . . 95

5.3 Experimental Approach _. . . . . . . . . . . . . . . . 98

5.4 Experimental Observations and Discussion . . . . . . . . . . . . . 101

5.4.1 Comparison of methods for determining the zero field $(n+$ 1) $d_{5 / 2}-n g$ intervals . . . . . . . . . . . . . . 101

5.4.2 The $(n+1) d_{5 / 2}-n g$ intervals and $n g$ quantum defects $\ldots 110$

5.4.3 The $(n+1) d_{5 / 2}-n h$ intervals and $n h$ quantum defects . . . 111

5.4.4 The $\mathrm{Rb}^{+}$ionic dipole and quadrupole polarizabilities . . . . . 114

5.5 Conclusion . . . . . . . . . . . . . . . . . . . . . . . . . 117

REFERENCES . . . . . . . . . . . . . . . . . . . 119

6 Future Work 123

6.1 Introduction . . . . . . . . . . . . . . . . . . . . . 123

$6.2 n s_{1 / 2} n s_{1 / 2} \rightarrow n p_{1 / 2} n p_{3 / 2}$ Transition $\ldots \ldots \ldots \ldots 123$

6.3 Formation of $(\mathrm{n}+2) \mathrm{p}$ atoms $\ldots \ldots \ldots \ldots \ldots \ldots \ldots$

REFERENCES . . . . . . . . . . . . . . . . . 138

$\begin{array}{lll}7 & \text { Conclusion } & 139\end{array}$ 


\section{List of Figures}

2.1 The energy levels involved with the magneto-optical trapping of $\mathrm{Ru}-$

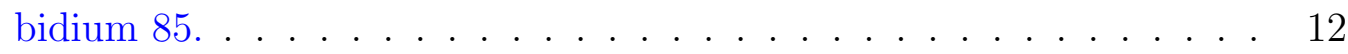

2.2 The magneto optical trap $\ldots \ldots \ldots \ldots \ldots$

2.3 The top view of the Littrow-configuration ECDL. The figure is adapted from Fig. 2.5 in $[5] \ldots \ldots \ldots \ldots \ldots \ldots$

2.4 The saturated absorption spectroscopy (SAS) signal used to frequency lock the lasers. The top spectrum is for the trap laser and the bottom spectrum is for the repump laser. The black vertical arrows represent the peaks that correspond to the transitions labeled below. The red slanted arrows represent the side-lock point. The figure is adapted from Fig. 2.7 in [5] . . . . . . . . . . . . . . . . . . . . . . 17

2.5 The image of trapped atoms and a Gaussian fit. . . . . . . . . . . 21 
2.6 Field ionization circuit diagram. It is taken from Fig. 2.17 of [4]. The parameters are R1: $8 \Omega, \mathrm{R} 2: 51.8 \Omega, \mathrm{R} 3: 100 \mathrm{k} \Omega$ (Optional), and $\mathrm{C} 1$ : $47 \mu \mathrm{F}$. CONN1, CONN2, and CONN3 are DC Voltage, trigger pulse, and output, respectively. The trigger transformer has a turns-ratio of 30.

2.7 Fast and slow field ionization pulses. The fast pulse has a risetime of

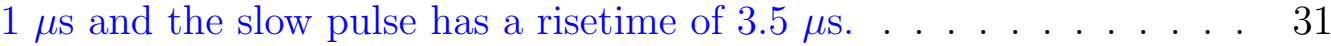

2.8 This figure shows the times at which the ionization signal appears as the excitation laser tuning with respect to the ionization limit of Rubidium is changed when ions are being detected. The slow field ionization pulse with a peak amplitude of $500 \mathrm{~V}$ was used to obtain this data. It is clear that at laser tuning of $-1750 \mathrm{GHz}$ and above, the ionization signals always appear at $5.7 \mu \mathrm{s}$. This shows that we lose state-selectivity of the states with high $n^{*} \ldots \ldots . \ldots 32$

2.9 This figure shows the times at which the ionization signal appears as the excitation laser tuning with respect to the ionization limit of Rubidium is changed when electrons are being detected. The slow field ionization pulse with a peak amplitude of $500 \mathrm{~V}$ was used to obtain this data. Different from ion detection, there is no loss of state-selectivity. . . . . . . . . . . . . . 
2.10 The timing diagram for a typical experiment. $T$ is the delay between the Rydberg excitation and the beginning of the microwave pulse. Typically, the microwave pulse is sent in immediately following the $480 \mathrm{~nm}$ excitation. The microwave pulse typically has a width that is on the order of $1 \mu \mathrm{s}$. The whole sequence repeats at $20 \mathrm{~Hz}$ frequency, which is the repetition rate of the $480 \mathrm{~nm}$ pulse. . . . . . . . . . . . 34

3.1 Energy levels for the $n d_{5 / 2} n d_{5 / 2} \rightarrow(n+1) d_{j}(n-2) f_{7 / 2}$ microwave transition, shown by the solid arrow. This transition is allowed due to the configuration interaction between the $n d_{5 / 2} n d_{5 / 2}$ and $(n+2) p_{3 / 2}(n-$ 2) $f_{7 / 2}$ state, shown by the broken double headed arrow. . . . . . . . . 38

3.2 AC Stark shift of the $(n+1) d_{j}(n-2) f$ and $(n+2) p_{3 / 2}(n-2) f_{7 / 2}$ states 43

3.3 Field ionization signal for the $39 d_{5 / 2} 39 d_{5 / 2} \rightarrow 40 d_{5 / 2} 37 f_{7 / 2}$ transition with microwaves off (broken line) and microwaves on (solid line). The microwaves are tuned to $33.613 \mathrm{GHz}$, the resonance frequency for $39 d_{5 / 2} 39 d_{5 / 2} \rightarrow 40 d_{5 / 2} 37 f_{7 / 2}$ transition. . . . . . . . . . . . . 48

3.4 Observed $39 d_{5 / 2} 39 d_{5 / 2} \rightarrow 40 d_{j} 37 f_{7 / 2}$ resonances for a range of microwave field amplitudes. The dotted lines represent the calculated resonance frequencies for the transitions at zero microwave power and $R=\infty \ldots \ldots \ldots \ldots \ldots \ldots$

3.5 Observed $44 d_{5 / 2} 44 d_{5 / 2} \rightarrow 45 d_{j} 42 f_{7 / 2}$ resonances for a range of microwave field amplitudes. The dotted lines represent the calculated resonance frequencies for the transitions at zero microwave power and $R=\infty \ldots \ldots \ldots \ldots \ldots \ldots \ldots$ 
3.6 Resonance frequency vs. microwave power for the $37 d_{5 / 2} 37 d_{5 / 2} \rightarrow$ $38 d_{5 / 2} 35 f_{7 / 2}$ transition. . . . . . . . . . . . . . . . 51

3.7 Observed $39 d_{5 / 2} 39 d_{5 / 2} \rightarrow 40 d_{j} 37 f_{7 / 2}$ resonances for a range of peak Rydberg atom densities. The dotted lines represent the calculated resonance frequencies for the transition at zero microwave power and

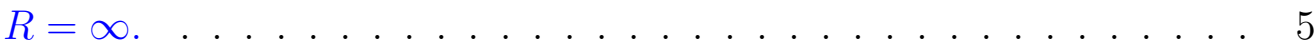

3.8 FPT vs. microwave field amplitude for $n=35(\triangle), n=37(+)$, $n=40(\circ)$, and $n=42(\square)$ at similar densities of $\rho_{0}=1.7 \times 10^{8} \mathrm{~cm}^{-3}$

3.9 FPT vs. $\frac{n^{6} \rho E}{\Delta}$ for $35 \leq n \leq 42 \ldots \ldots \ldots$

3.10 Energy level diagram for the $39 d_{5 / 2} 39 d_{5 / 2} \rightarrow 41 p_{3 / 2} 38 d_{5 / 2}$ transition . 56

3.11 Observed $39 d_{5 / 2} 39 d_{5 / 2}$ to $41 p_{3 / 2} 38 d_{5 / 2}$ transition . . . . . . . . . . . 57

4.1 One, two, and three photon microwave transitions from $n d_{5 / 2} n d_{5 / 2}$ state. The transitions to $(n+3) p_{j}(n-2) f$ states are two-photon transitions, and the transition to $(n+4) s_{1 / 2}(n-2) f$ state is a threephoton transition. The diagram is approximately to scale. . . . . . . 63

4.2 Floquet energy levels for $\mathrm{n}=39$ as a function of the microwave frequency for zero microwave field amplitude. No dipole-dipole interaction is included. . . . . . . . . . . . . . . . . .

4.3 Floquet energy levels for $\mathrm{n}=39$ as a function of the microwave frequency for microwave field amplitude of $415 \mathrm{mV} / \mathrm{cm}$. No dipole-dipole interaction is included. . . . . . . . . . . . . . . . . 
4.4 An expanded view of the region marked by dashed box in Fig. 4.2 and Fig. 4.3 containing the two and three photon Forster resonances. Solid lines and dotted lines represent microwave coupling of 0 and 200 $\mathrm{MHz}$, respectively. For $W_{42 p_{3 / 2} 37 f}-2 \omega$, the solid line and the dotted line overlap. . . . . . . . . . . . . . . . . . 71

4.5 The observed one photon transitions originating from $n d_{5 / 2} n d_{5 / 2}$. The $(n+2) p_{3 / 2}(n-2) f$ state is nearly degenerate with $n d_{5 / 2} n d_{5 / 2}$ level. For $n=39,(n+2) p_{3 / 2}(n-2) f$ level is detuned by $477.8 \mathrm{MHz}$. . . . 76

4.6 Observed $40 d_{5 / 2} 40 d_{5 / 2} \rightarrow 43 s_{1 / 2} 38 f_{7 / 2}$ resonances for a range of microwave field amplitudes. The calculated resonance frequency for the transition at zero microwave power and $R=\infty$ is $53.721 \mathrm{GHz}$. . . . 78

4.7 Fractional Population Transfer (FPT) vs. microwave field amplitude for the $40 d_{5 / 2} 40 d_{5 / 2} \rightarrow 43 s_{1 / 2} 38 f_{7 / 2}$ transition $\ldots \ldots . . . . . .79$

4.8 Observed resonance for the $41 d_{5 / 2} 41 d_{5 / 2} \rightarrow 43 p_{3 / 2} 39 g$ transition. The peak is shifted to higher frequency by $1.4 \mathrm{MHz}$ due to AC Stark shift. The calculated frequency for the transition is $1190.4 \mathrm{MHz}$. . . . . . . 81

4.9 One photon transition originating from $n s_{1 / 2} n s_{1 / 2}$ that was observed.

4.10 Observed resonance for $40 s_{1 / 2} 40 s_{1 / 2} \rightarrow 39 d_{5 / 2} 39 p_{3 / 2}$ transition. The calculated frequency for the transition is $31.441 \mathrm{GHz}$. The peak is not observably shifted. . . . . . . . . . . . . . 84 
4.11 Observed $39 d_{5 / 2} 39 d_{5 / 2} \rightarrow 42 p_{1 / 2} 37 f_{7 / 2}$ resonances for a range of microwave field amplitudes. The calculated two photon resonance frequency for the transition at zero microwave power and $R=\infty$ is $110.273 \mathrm{GHz} . \ldots \ldots . \ldots . \ldots . \ldots . \ldots 86$

4.12 Fractional Population Transfer (FPT) vs. microwave field amplitude squared for the $39 d_{5 / 2} 39 d_{5 / 2} \rightarrow 42 p_{1 / 2} 37 f_{7 / 2}$ transition . . . . . . . . 87

4.13 Observed $39 d_{5 / 2} 39 d_{5 / 2} \rightarrow 42 p_{3 / 2} 37 f_{7 / 2}$ resonances for a range of microwave field amplitudes. The calculated two photon resonance frequency for the transition at zero microwave power and $R=\infty$ is $111.699 \mathrm{GHz} \ldots \ldots \ldots \ldots 8 . \ldots \ldots$

4.14 The observed resonance for $40 d_{5 / 2} 40 d_{5 / 2} \rightarrow 44 s_{1 / 2} 38 f$ transition. The peak is shifted to higher frequency by $25 \mathrm{MHz}$ due to AC Stark shift.

5.1 MOT configuration of this experiment. . . . . . . . . . . . . 100

5.2 Schematic of the Rydberg energy levels of this experiment. . . . . . . 100

5.3 The frequency of the microwave $29 d_{5 / 2} \rightarrow 28 g$ transition vs bias electric field in the $z$ direction. The maximum frequency is 104 371.08(40) $\mathrm{MHz}$ at a bias voltage of $V_{b}=V_{0}=0.24 \mathrm{~V}$. At this bias, $E_{z}$ is nulled. 102

5.4 (a) The $29 d_{5 / 2} \rightarrow 28 k$ Stark spectrum at bias voltage of $V_{b}=-1.3 \mathrm{~V}$ at the relative microwave power 1 . The high $k$ states are indicated in the graph. (b) The $29 d_{5 / 2} \rightarrow 28 g$ transition at the same bias field as (a) but a relative microwave power of 0.032 . . . . . . . . . . . . . 104 
5.5 The $29 d_{5 / 2} \rightarrow 28 \mathrm{~g}$ transition frequency vs the square of the Stark splitting of the high $k$ states $\left(\Delta \nu_{S}^{2}\right)$ obtained from Stark spectroscopy. At $\Delta \nu_{S}^{2}=0$, the stray field is zero and the $29 d_{5 / 2} \rightarrow 28 \mathrm{~g}$ transition frequency is $104378.9(62) \mathrm{MHz}$. . . . . . . . . . . . . . . 105

5.6 (a) The observed the $29 d_{5 / 2} \rightarrow 28 \mathrm{~g}$ resonance signals with different bias voltages. As shown by the slanted line, the signal amplitude varies linearly with the observed resonance frequency. The resonance frequency increases and the signal amplitude decreases as the bias voltage is reduced from $V_{b}=0.61 \mathrm{~V}$ to $0.34 \mathrm{~V}$, which reduces $E_{z}$ from $150 \mathrm{mV} / \mathrm{cm}$ to $41 \mathrm{mV} / \mathrm{cm}$. (b) The $29 d_{5 / 2} \rightarrow 28 \mathrm{~g}$ microwave transition frequency as a function of the relative $28 \mathrm{~g}$ signal amplitude. From the graph, the $29 d_{5 / 2} \rightarrow 28 g$ transition frequency at zero stray field is 104 372.70(28) MHz. ....................... 107

5.7 The graph of the relative $28 \mathrm{~g}$ signal amplitude $(S)$ as a function of squared voltage $\left(V_{b}-V_{0}\right)^{2}$ and squared static field $E_{z}^{2}$ in the $z$ direction of the system. Since $S=a\left(E_{z}^{2}+E_{\perp}^{2}\right)$, from the intercept and slope of the graph we determine $E_{\perp}$ to be $91 \mathrm{mV} / \mathrm{cm} . \quad$. . . . . . . . . . . 109

5.8 The observed amplitude as the $29 d_{5 / 2} \rightarrow 28 h$ resonance changes in different bias voltages. . . . . . . . . . . . . . . . . . 112

5.9 The frequency of the observed $29 d_{5 / 2} \rightarrow 28 h$ resonances as a function of the square root of the relative $28 h$ signal amplitude, $\sqrt{S}$. From the graph, the $29 d_{5 / 2} \rightarrow 28 h$ transition frequency at zero stray field is 105

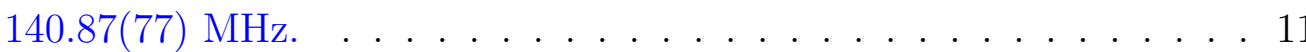


5.10 A plot of the measured $n g$ and $n h$ quantum defects scaled by $n^{3}\left\langle r_{n \ell}^{-4}\right\rangle / 2$ vs $\left\langle r_{n \ell}^{-6}\right\rangle /\left\langle r_{n \ell}^{-4}\right\rangle$ using Eq. (5.14). There are 3 data points for the $n h$ quantum defects, $28 \leq n \leq 30$, and 4 data points for the $n g$ quantum defects, $27 \leq n \leq 30$. A fit to the straight line yields the y-intercept and slope, which are $\alpha_{d}$ and $\alpha_{q}$, respectively. The resulting fit values are $\alpha_{d}=9.12(2) a_{0}^{3}$ and $\alpha_{q}=14(3) a_{0}^{5}$. . . . . . . . . . . . . 116

6.1 The figure shows the 39p signal at high (blue trace) and low (black trace) microwave power as the microwave frequency is swept across the resonance frequencies of the two atomic transitions $39 s_{1 / 2} \rightarrow 39 p_{1 / 2}$ and $39 s_{1 / 2} \rightarrow 39 p_{3 / 2}$. The Rydberg atom density is kept the same. At high microwave power, there is a new peak at $67.474 \mathrm{GHz}$. . . . . . . 124

6.2 The figure shows the 39p signal at high (blue trace) and low (black trace) Rydberg atom densities as the microwave frequency is swept across the resonance frequencies of the two atomic transitions $39 s_{1 / 2} \rightarrow$ $39 p_{1 / 2}$ and $39 s_{1 / 2} \rightarrow 39 p_{3 / 2}$. The microwave power is kept the same. At high density, there is a new peak at $67.474 \mathrm{GHz}$. . . . . . . . . . . 124

6.3 FPT vs. power for the $39 s_{1 / 2} 39 s_{1 / 2} \rightarrow 39 p_{1 / 2} 39 p_{3 / 2}$ transition. . . . . 125

6.4 FPT vs. density for the $39 s_{1 / 2} 39 s_{1 / 2} \rightarrow 39 p_{1 / 2} 39 p_{3 / 2}$ transition. . . . . 126

6.5 The resonant peaks at different densities. The "wing" on the lower frequency side is clearly visible. . . . . . . . . . . . . . . . . 129

6.6 Time resolved field ionization signals from a sample of atoms excited to the $42 d$ state at high and low density. Ions are being detected. . . 130 
6.7 The ratio of the "apparent" $(n+2) p$ signal vs. time integrated signal as the density of Rydberg atoms is changed. . . . . . . . . . . . . . . 132

6.8 The ratio of the "apparent" $(n+2) p$ signal vs. time integrated signal as the delay of the field ionization pulse is changed. . . . . . . . . . 133

6.9 The ionization signal from a sample of atoms excited to $42 d$ state with microwave turned on and off. Microwave frequency is tuned to the transition frequency of the two photon transition $44 p_{3 / 2} \rightarrow 45 p_{3 / 2}$. 135

6.10 The resonant peak for the $44 p_{3 / 2} \rightarrow 45 p_{3 / 2}$ transition. The observed transition frequency, from the Lorentzian fit, is $44.872 \mathrm{GHz}$ which shows a good agreement with the calculated frequency of $44.870 \mathrm{GHz}$. 136 


\section{List of Tables}

1.1 The scaling for some of the properties of Rydberg atoms . . . . . . . 2

1.2 The quantum defects of ${ }^{85} \mathrm{Rb}$. The values are taken from Li[7], Han[4], and Lee[6]. The fine structure splitting of $n g$ and $n h$ states could not be resolved. ............................ 3

2.1 The list of dyes used for dye amplification stages . . . . . . . . . 25

2.2 The list of microwave frequency multipliers used throughout the experiments in this dissertation. Pacific Millimeter V2 and W3WO need to be used in conjunction with an active doubler (Narda DBS 2640X220). 27

3.1 Energy detunings $(\Delta)$ between $n d_{5 / 2} n d_{5 / 2}$ and $(n+2) p_{3 / 2}(n-2) f_{7 / 2}$.

3.2 Resonance frequencies for the $n d_{5 / 2} n d_{5 / 2} \rightarrow(n+1) d_{5 / 2}(n-2) f_{7 / 2}$

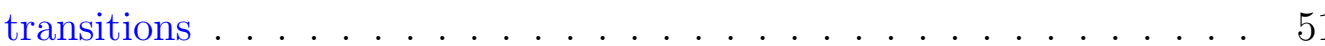

3.3 Resonance frequencies for the $n d_{5 / 2} n d_{5 / 2} \rightarrow(n+1) d_{3 / 2}(n-2) f_{7 / 2}$

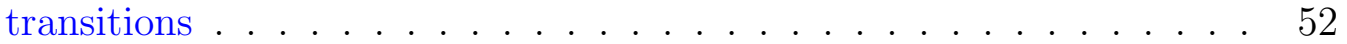

4.1 Microwave field amplitude required to produce $a_{1}=0.05 \ldots$. . . . . . 75 
4.2 Resonance frequencies and AC Stark shift values for one photon transitions. Calculated shifts are obtained from our Floquet model. The estimated maximum field amplitudes are calculated from the maximum observed shifts and calculated shifts. As a point of comparison, the unattenuated microwave field amplitude with at the center of the trap is estimated to be $693 \mathrm{mV} / \mathrm{cm}$ by using the following parameters: Output power of the active doubler/quadrupler $=15 \mathrm{dBm}$, gain of the horn antenna $=20 \mathrm{dBi}$, distance from the horn to the center of trap $=0.2 \mathrm{~m} \ldots \ldots \ldots \ldots \ldots \ldots \ldots$

4.3 Resonance frequencies and AC Stark shift values for two photon transitions. Calculated shifts are obtained from our Floquet model. The estimated maximum field amplitudes are calculated from the maximum observed shifts and calculated shifts. As a point of comparison, the unattenuated microwave field amplitude with at the center of the trap is estimated to be $693 \mathrm{mV} / \mathrm{cm}$ by using the following parameters: Output power of the active doubler/quadrupler $=15 \mathrm{dBm}$, gain of the horn antenna $=20 \mathrm{dBi}$, distance from the horn to the center of trap $=0.2 \mathrm{~m} \ldots \ldots \ldots \ldots \ldots$ 
4.4 Resonance frequencies and AC Stark shift values for three photon transition. Calculated shifts are obtained from our Floquet model. The estimated maximum field amplitudes are calculated from the maximum observed shifts and calculated shifts. As a point of comparison, the unattenuated microwave field amplitude with at the center of the trap is estimated to be $693 \mathrm{mV} / \mathrm{cm}$ by using the following parameters: Output power of the active doubler/quadrupler $=15 \mathrm{dBm}$, gain of the horn antenna $=20 \mathrm{dBi}$, distance from the horn to the center of trap $=0.2 \mathrm{~m} \ldots \ldots \ldots \ldots$

5.1 The $(n+1) d_{5 / 2} \rightarrow n g$ microwave transition frequencies in zero stray electric field and the extracted quantum defects of $n g$. . . . . . . . . 111

5.2 The $(n+1) d_{5 / 2} \rightarrow n h$ microwave transition frequencies in zero stray electric field and the extracted quantum defects of $n h . \quad$. . . . . . 113

5.3 The $\mathrm{Rb}^{+}$dipole $\left(\alpha_{d}\right)$ and quadrupole $\left(\alpha_{q}\right)$ polarizabilities obtained from this work and other theoretical (Th) and experimental (Exp) results. . . . . . . . . . . . . . . . . . . 117 


\section{Chapter 1}

\section{Introduction}

\subsection{Rydberg Atoms}

Atoms that are excited to a very high principal quantum number $(n>10)$ are called "Rydberg Atoms"[3]. What makes them such an interesting object of study is the fact that many of their physical properties such as size, dipole moment, and geometric cross section are greatly exaggerated compared to ground state atoms. For this reason, Rydberg atoms are easily perturbed or ionized by collisions or external fields and interact strongly with each other even at the $\mu \mathrm{m}$ scale. Table 1.1 shows how various properties of Rydberg atoms scale with respect to the principal quantum number.

Rubidium (more specifically, ${ }^{85} \mathrm{Rb}$ ) is the atomic species used exclusively for the work in this dissertation. Rubidium belongs to the Alkali metal group, which has one valence electron. When an alkali metal such as Rubidium is excited to a Rydberg 


\begin{tabular}{cc}
\hline \hline Property & Scaling \\
\hline Orbital Radius & $n^{2}$ \\
Dipole Moment & $n^{2}$ \\
Lifetime & $n^{3}$ \\
Geometric Cross Section & $n^{4}$ \\
Spacing between Adjacent Levels & $n^{3}$ \\
Binding Energy & $1 / n^{2}$ \\
\hline \hline
\end{tabular}

Table 1.1: The scaling for some of the properties of Rydberg atoms

state, the valence electron is located far away from the core and it sees a core with one positive unit charge, and this results in Rydberg atoms having similar properties to those of the hydrogen atom.

For low angular momentum states, however, the electron orbits are elliptical, and the energies deviate from the hydrogenic levels due to core penetration and polarization. A correction term, called the quantum defect $\left(\delta_{n \ell j}\right)$, needs to be included in the expression for the binding energy of a hydrogenic atom to account for the energy shift associated with the core penetration and polarization. In other words, the following expression for the hydrogenic energy

$$
E=-\frac{\mathrm{Ry}}{n^{2}}
$$

needs to be modified to

$$
E=-\frac{\mathrm{Ry}}{\left(n-\delta_{n \ell j}\right)^{2}}
$$

where Ry is the Rydberg constant which has a value of $109736.605 \mathrm{~cm}^{-1}$ for Rubidium. The quantity $n^{*}=n-\delta_{n \ell j}$ is called effective principal quantum number.

The value of $\delta_{n \ell j}$ depends strongly on $\ell$ and weakly on $n$ and $j$. The quantum 


\begin{tabular}{cc}
\hline \hline State & Quantum Defect \\
\hline$s_{1 / 2}$ & 3.1312 \\
$p_{1 / 2}$ & 2.6549 \\
$p_{3 / 2}$ & 2.6417 \\
$d_{3 / 2}$ & 1.3481 \\
$d_{5 / 2}$ & 1.3465 \\
$f_{5 / 2}$ & 0.01652 \\
$f_{7 / 2}$ & 0.01654 \\
$g$ & 0.00397 \\
$h$ & 0.00141 \\
\hline \hline
\end{tabular}

Table 1.2: The quantum defects of ${ }^{85} \mathrm{Rb}$. The values are taken from Li[7], Han[4], and Lee[6]. The fine structure splitting of $n g$ and $n h$ states could not be resolved.

defect increases as $\ell$ decreases because the electron orbit becomes more elliptical and the effect of core penetration and polarization gets stronger. For $\ell>3$, the quantum defect rapidly decreases.

The quantum defects can be empirically determined by measuring energy intervals between states. Previous students in this group measured quantum defects for the s, $\mathrm{p}, \mathrm{d}$, and f states using microwave spectroscopy $[4,7]$. The measurement of quantum defects for the $\mathrm{g}$ and $\mathrm{h}$ states is a part of this dissertation (Chapter 5). Table 1.2 lists the quantum defects of $\mathrm{Rb}$ that have been determined to date.

Although various properties of Rydberg atoms have been extensively studied for decades, the advent of magneto optical trap (MOT) opened up new possibilities by allowing experimenters to create a cold, dense sample of Rydberg atoms that is often referred to as a "frozen" Rydberg gas. The sub $m K$ temperature of the Rydberg atoms in a MOT leads to the atoms only moving a small fraction of their interatomic spacing on average during the typical experimental time scale of $1 \mu \mathrm{s}$. The typical 
density of $10^{8}-10^{9} \mathrm{~cm}^{-3}$ results in a dipole-dipole coupling that is on the order of $1 \mathrm{MHz}$. The strong interaction among the essentially static Rydberg atoms has led to observations of many phenomena that could not be observed with isolated atoms such as the spontaneous evolution of Rydberg atoms into a cold plasma[10], Forster resonant energy transfer[1, 2, 11], broadening of atomic resonances due to dipoledipole interaction[9], dipole blockade[8], and microwave transitions between pairs of Rydberg atoms[5, 12].

In particular, the microwave transitions between pairs of Rydberg atoms are one of the main topics investigated this dissertation.

\subsection{Thesis Outline}

The rest of this dissertation is structured as follows.

In Chapter 2, an overview of the experimental apparatus and technique used throughout this dissertation is given. The chapter is broken down into sections which correspond to the major steps involved in conducting the experiment. In addition to the principle of operation for various parts of the apparatus, measurement results for the purpose of calibrating or characterizing the apparatus are provided when applicable. Needless to say, it is crucial for an experimenter to be thoroughly familiar with the principles behind the apparatus, their operation, as well as troubleshooting before one can run experiments without interruptions. It takes both patience and time to become proficient in the operation and troubleshooting of the apparatus.

Chapter 3 reports the systematic study of the microwave transition, $n d_{5 / 2} n d_{5 / 2} \rightarrow$ 
$(n+1) d_{j}(n-2) f$, between pairs of Rb Rydberg atoms. The primary goal of the experiment was to test the scaling of fractional population transfer (FPT) for the aforementioned transition with respect to the principal quantum number, microwave field amplitude, and density. The scaling of FPT was found to agree well with predictions of a simple theoretical model based on the configuration interaction (CI). The zero-field resonance frequencies were obtained as well and are in excellent agreement with the theoretical frequencies for $R=\infty$.

Chapter 4 reports a study that is an extension of the experiment described in Chapter 3. More specifically, it reports on the observation of single and multi photon transitions involving pairs of Rb Rydberg atoms that are allowed due to the same configuration interaction as in $n d_{5 / 2} n d_{5 / 2} \rightarrow(n+1) d_{j}(n-2) f$. It is shown that the transitions can be described as Forster resonant energy transfers between Floquet states. The Floquet-Forster model reduces to the CI model used previously.

Chapter 5 reports an experimental technique which was used to determine the zero field intervals between high $\ell$ states of $\mathrm{Rb}$ in a magneto-optical trap in spite of the fact that the stray electric field can only be controlled in one direction. The technique was used to determine the zero field $(n+1) d_{5 / 2}-n g$ and $(n+1) d_{5 / 2}-n h$ intervals. The quantum defects of the $n g$ and $n h$ states are determined from the intervals. Lastly, using the quantum defects and the adiabatic core polarization theory, the ionic dipole and quadrupole polarizabilities of $R b^{+}$are determined. 


\section{REFERENCES}

[1] Anderson, W. R., Veale, J. R., \& Gallagher, T. F. (1998). Resonant dipole-dipole energy transfer in a nearly frozen rydberg gas. Phys. Rev. Lett., 80, 249-252.

[2] Bohlouli-Zanjani, P., Petrus, J. A., \& Martin, J. D. D. (2007). Enhancement of rydberg atom interactions using ac stark shifts. Phys. Rev. Lett., 98, 203005.

[3] Gallagher, T. F. (1994). Rydberg Atoms. Cambridge, England: Cambridge University Press.

[4] Han, J., Jamil, Y., Norum, D. V. L., Tanner, P. J., \& Gallagher, T. F. (2006). $\mathrm{Rb} n f$ quantum defects from millimeter-wave spectroscopy of cold ${ }^{85} \mathrm{Rb}$ rydberg atoms. Phys. Rev. A, 74, 054502.

[5] Lee, J. \& Gallagher, T. F. (2016). Microwave transitions from pairs of rb $n d_{5 / 2} n d_{5 / 2}$ atoms. Phys. Rev. A, 93, 062509.

[6] Lee, J., Nunkaew, J., \& Gallagher, T. F. (2016). Microwave spectroscopy of the cold rubidium $(n+1) d_{5 / 2} \rightarrow n g$ and $n h$ transitions. Phys. Rev. A, 94, 022505.

[7] Li, W., Mourachko, I., Noel, M. W., \& Gallagher, T. F. (2003). Millimeter-wave spectroscopy of cold rb rydberg atoms in a magneto-optical trap: Quantum defects of the ns, np, and nd series. Phys. Rev. A, 67, 052502.

[8] Lukin, M. D., Fleischhauer, M., Cote, R., Duan, L. M., Jaksch, D., Cirac, J. I., \& Zoller, P. (2001). Dipole blockade and quantum information processing in mesoscopic atomic ensembles. Phys. Rev. Lett., 87, 037901.

[9] Park, H., Tanner, P. J., Claessens, B. J., Shuman, E. S., \& Gallagher, T. F. (2011). Dipole-dipole broadening of rb $n s-n p$ microwave transitions. Phys. Rev. A, 84, 022704.

[10] Robinson, M. P., Tolra, B. L., Noel, M. W., Gallagher, T. F., \& Pillet, P. (2000). Spontaneous evolution of rydberg atoms into an ultracold plasma. Phys. Rev. Lett., 85, 4466-4469.

[11] van Ditzhuijzen, C. S. E., Koenderink, A. F., Hernández, J. V., Robicheaux, F., Noordam, L. D., \& van den Heuvell, H. B. v. L. (2008). Spatially resolved observation of dipole-dipole interaction between rydberg atoms. Phys. Rev. Lett., $100,243201$. 
[12] Yu, Y., Park, H., \& Gallagher, T. F. (2013). Microwave transitions in pairs of rb rydberg atoms. Phys. Rev. Lett., 111, 173001. 


\section{Chapter 2}

\section{Experimental Approach}

Many instruments were used for the experiments described in this dissertation. As is true for any experimental work, it is crucial to understand all components of the apparatus and become proficient at operating and maintaining them to be able to run experiments. In this chapter, an overview of the experimental apparatus used in this dissertation is provided. Experiment-specific descriptions or modifications will be given in the later chapters.

In most cases, an experiment consists of the following for major steps. First, a sample of cold rubidium atoms is prepared in a Magneto Optical Trap (MOT). Second, the trapped atoms are excited to a Rydberg state. Third, the Rydberg atoms are manipulated with either microwaves or static electric fields. Lastly, the atoms are ionized by state-selective field ionization, and the resulting ions or electrons are pushed to the multichannel plate (MCP) detector. The signal from the MCP detector is sent to the computer for later analysis. Accordingly, this chapter is broken 
down into the following sections: 1) Magneto Optical Trap, 2) Excitation of Rydberg Atoms, 3) Microwave Setup, and 4) Detection and Data Acquisition. Since theoretical and experimental descriptions of Magneto-Optical Traps are widely available, only brief descriptions will be given. There are excellent references if the reader wishes to learn in more depth about these topics. For instance, Metcalf[7] gives a good theoretical description. For experimental descriptions including a more detailed description on this particular setup, the dissertations of previous students (Anderson[1], Li[5], Han[2], Park[8], Levac[4]) who worked on the setup can be consulted.

\subsection{Magneto Optical Trap (MOT)}

A magneto-optical trap is an apparatus used to make a sample of extremely cold trapped neutral atoms. It uses a combination of laser (that provides damping force) and magnetic fields (that provides trapping force) to trap neutral atoms. Since it was first realized in 1987, the Magneto-Optical Trap (MOT) has been one of the most important tools in experimental atomic physics. Its popularity arises from its simple and relatively inexpensive construction, easy operation, and robust performance. Its small size does not require much space, allowing table-top experiments. The MOT used in this dissertation is a vapor-loaded type, in which the slowly moving atoms of rubidium vapor, produced by heating a rubidium getter, are captured by a combination of laser and magnetic field. 


\subsubsection{Principle of Operation}

The following two mechanisms are responsible for the operation of a MOT: Doppler cooling and magnetic trapping. Doppler cooling is based on the law of momentum conservation. When an atom and a photon interact, the momentum has to be conserved. If an atom absorbs a photon propagating along a fixed direction (i.e. the laser propagation direction), the atom gains a momentum in the direction the photon was moving before absorption. By red detuning a laser beam, it is possible to pick out and slow down only the atoms that are moving toward the laser (with a specific velocity range), because only those atoms will see the correct up-shifted frequency due to the Doppler effect. Since the atom will emit a photon in an arbitrary direction, when repeated many times this process of absorption-spontaneous emission results in a friction force that slows down the atom. In order to cool atoms, the slowing down process must occur in all three Cartesian axes. This is achieved by sending in 3 orthogonal laser beams through the center of the trap and then reflecting the beams back along the same direction.

Magnetic trapping is based on the Zeeman shift, which is the shifting and splitting of $m_{f}$ energy levels in a presence of magnetic field. In MOT, a pair of anti Helmholtz coils creates a spatially varying quadrupole magnetic field that is zero at the center of the trap and increases linearly in all directions. As a result, the atom that moves away from the center of the MOT experiences an energy shift that makes the atom more

likely to absorb red-detuned photons. Different $m_{f}$ states will interact differently with the two possible circular polarizations of the laser. It is important to ensure that the correct polarizations are used so that the atoms are always pushed toward 
the center.

In order to cool the atoms by using a laser, the atom must go through many cycles of absorption-spontaneous emission. For the atom to be able to repeatedly go through this cycle, it needs to have a specific energy level structure known as a closed optical loop. For ${ }^{85} \mathrm{Rb}, 5 s_{1 / 2}, F=3$ and $5 p_{3 / 2}, F=4$ states form such a loop. Due to the detuning of the laser, however, there is a nonzero probability of the laser exciting the $5 s_{1 / 2}, F=3$ state to the $5 p_{3 / 2}, F=3$ state. When this happens, the atom may decay from $5 p_{3 / 2}, F=3$ to the $5 s_{1 / 2}, F=2$ state, which is inaccessible to the laser, and the atom falls out of the cooling/trapping cycle. To put these atoms back into the cooling/trapping cycle, another "repump" laser that is tuned to the $5 s_{1 / 2}, F=2 \rightarrow 5 p_{3 / 2}, F=3$ transition is employed. The energy levels involved with the magneto-optical trapping of rubidium 85 are shown in Fig. 2.1. The laser that is tuned to $5 s_{1 / 2}, F=3 \rightarrow 5 p_{3 / 2}, F=4$ will be referred to as the "trap" laser. The wavelength of the trap laser is $780.030 \mathrm{~nm}$, and the wavelength of the repump laser is $780.024 \mathrm{~nm}$.

\subsubsection{Vacuum Chamber}

Since the collisions between the trapped atom and background gas can kick the trapped atom out of the trap, the trapping can only be achieved in a vacuum chamber kept in ultra-high vacuum (UHV) condition. We use the MCF-600-SS200408-A vacuum chamber from Kimball Physics to house the core components of the MOT setup including the rubidium dispenser, electrodes for field ionization and applying static fields (when necessary), and micro-channel plate detector. The chamber has 


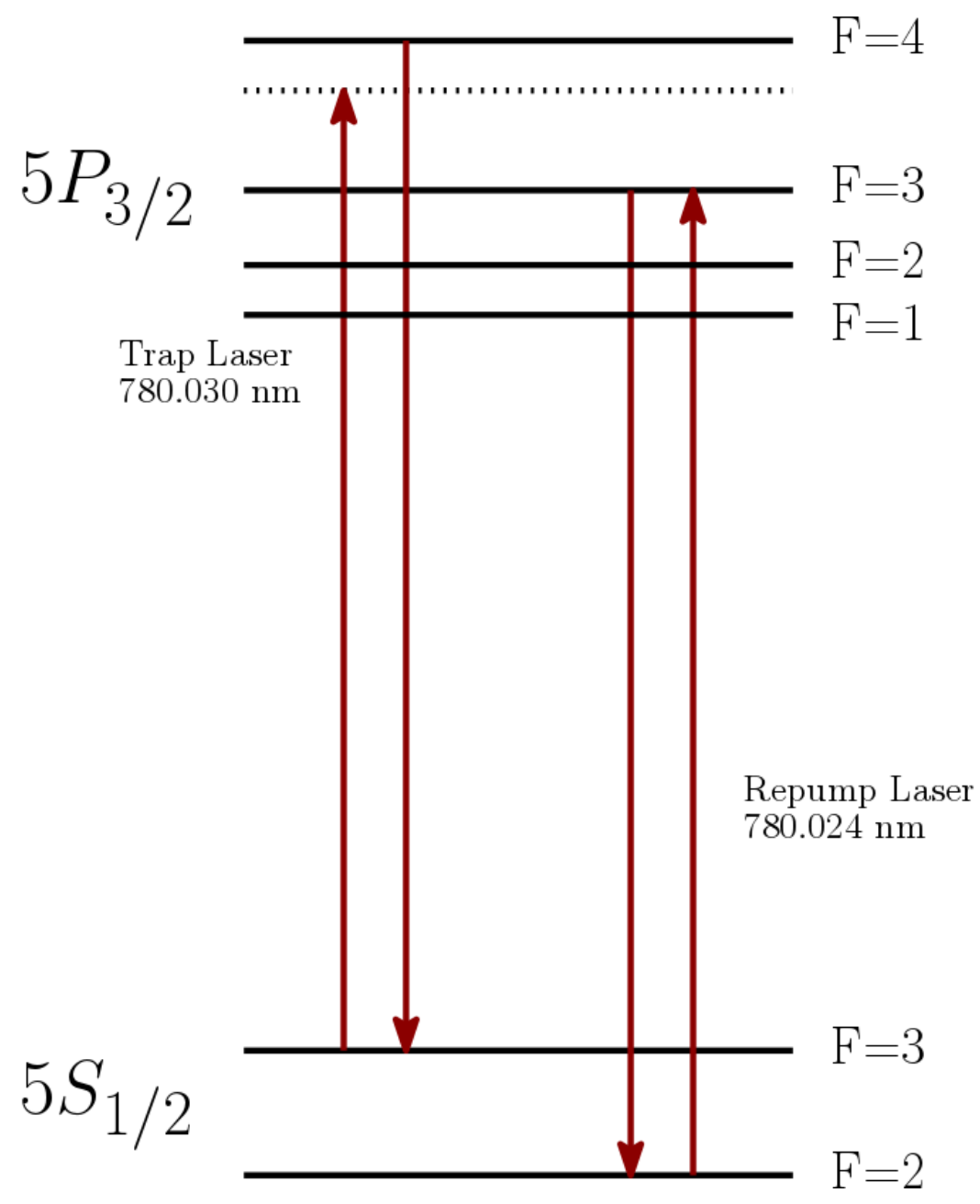

Figure 2.1: The energy levels involved with the magneto-optical trapping of Rubidium 85 . 
various windows and flanges that provide optical access and allow ion pump and detector to be connected. The pressure is constantly kept below $10^{-8}$ torr by the ion pump manufactured by Physical Electronics. The rubidium atoms are supplied by a $\mathrm{Rb}$ dispenser (or Rb getter) manufactured by SAES. When a current is passed through the getter, the dispenser releases the Rb atoms, among which the slow moving ones are trapped by the laser and magnetic field. The typical value of current supplied to the getter is about $4 \mathrm{~A}$, although higher current is needed as the getters age. There are four vertical stainless steel rods at the center of the vacuum chamber. The rods are $1.65 \mathrm{~mm}$ in diameter and pass through the corners of a horizontal square $18 \mathrm{~mm}$ on a side. The two rods opposite the MCP detector are connected together (inside the vacuum chamber) and are used primarily to apply a field ionization pulse, although a DC bias voltage can also be applied if necessary. The two rods closest to the MCP are also connected together and can be grounded or biased to provide a static field. In the middle of the four rods where the trap forms the electric field produced by the pair of rods can be approximated as uniform.

\subsubsection{Electric Field}

When a DC bias voltage is applied to the rods, it results in an electric field in the interaction region where the field amplitude linearly depends on the applied bias voltage, i.e. $E=\alpha V$ where $\alpha$ is a constant. The value of $\alpha$ can be experimentally determined by comparing the DC Stark shifts of resonant peak of an atomic transition $n s \rightarrow n p_{1 / 2}$ at different values of DC bias voltage against the static polarizabilities of the states involved in the transition. The static polarizabilities can be found in 


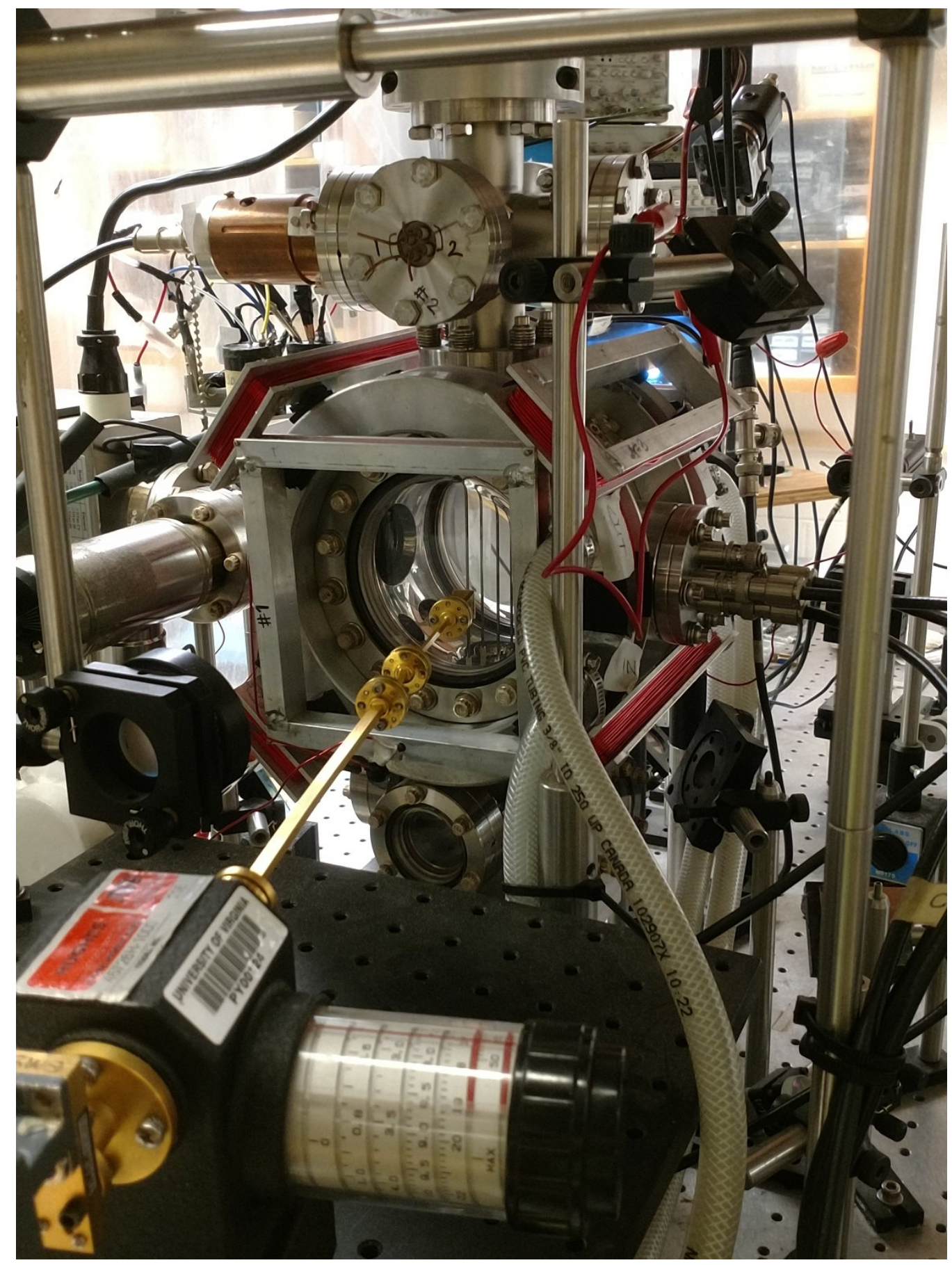

Figure 2.2: The magneto optical trap 
Table 2.2 of J. Pritchard's thesis[9]. Using this method, the value of $\alpha$ is determined to be $0.3324(\mathrm{~V} / \mathrm{cm}) / \mathrm{V}$.

\subsubsection{External Cavity Diode Laser (ECDL)}

As mentioned in section 2.1.1, two lasers (the trap laser and repump laser) with operating wavelengths near $780 \mathrm{~nm}$ are required to trap Rb atoms in the MOT. Two homemade cw Littrow-configuration external cavity diode lasers (ECDL) are used for this purpose. Diode lasers do not take up much space, are inexpensive, stable (under the right conditions), and fairly straightforward to construct. The external cavity can reduce the linewidth to below $1 \mathrm{MHz}$. There are many helpful articles on the use of diode lasers in atomic physics experiments[6, 10]. Fig. 2.3 shows the top view of our Littrow-configuration ECDL. The laser diode that we use is QPhotonics QLD-780-80S which has a typical output power of $80 \mathrm{~mW}$ and an output wavelength that is centered at $782 \mathrm{~nm}$. A collimation tube with optics (Thorlabs LT110P-B) houses the laser diode. The collimation is achieved by rotating the lens in front of the tube. The collimated output beam is directed toward the diffraction grating. The first order reflection from the grating is directed back toward the diode. The grating and the highly reflective back facet of the diode form the external cavity. The zeroth order reflection is taken as the output. The grating is mounted on a mirror mount so that the grating angle can be adjusted. A piezoelectric actuator (Thorlabs AE0203D08F) is placed between the adjusting screw and the front plate to fine-tune the grating angle. By adjusting the angle between the incident beam and the grating, wavelength tuning can be achieved. The parts shown in Fig. 2.3 are fixed 


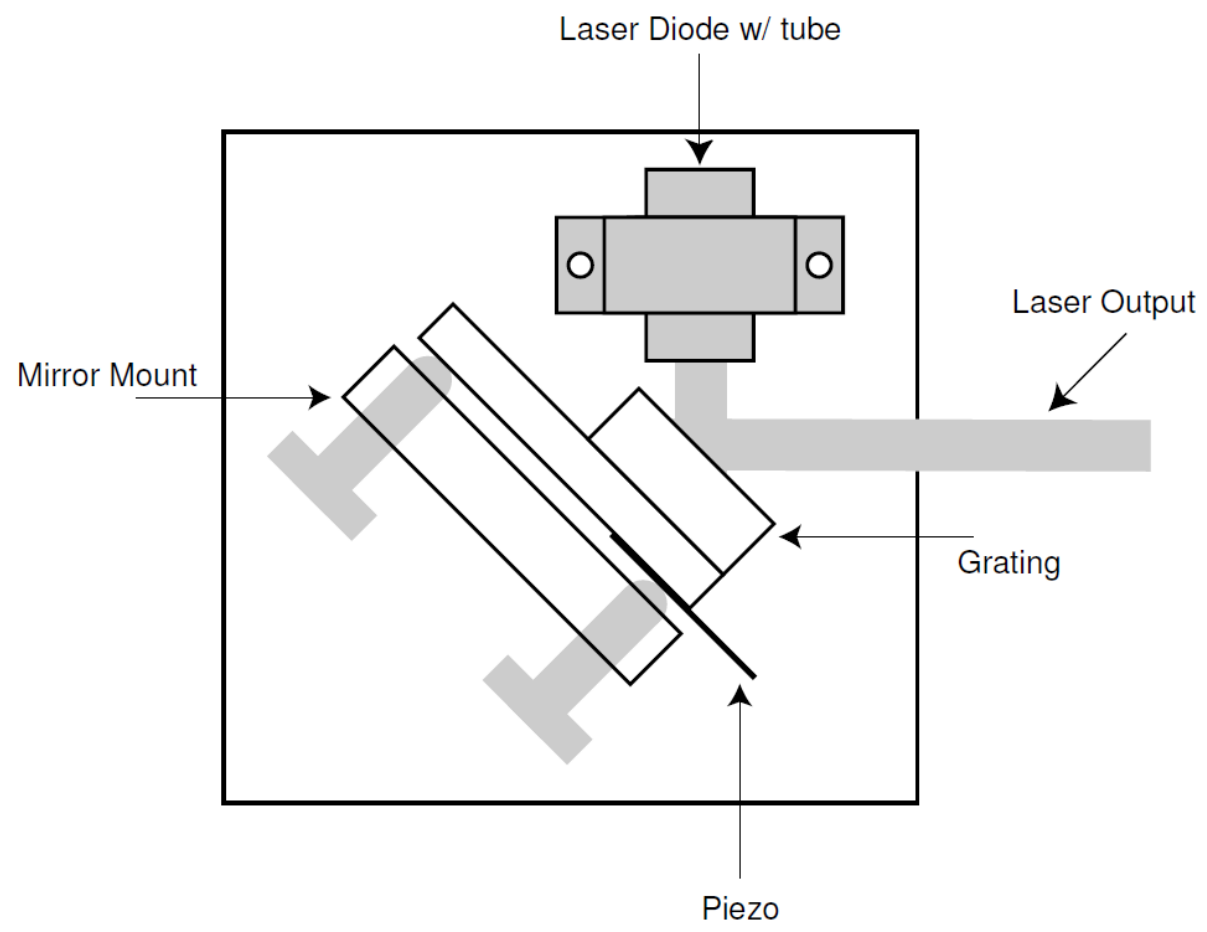

Figure 2.3: The top view of the Littrow-configuration ECDL. The figure is adapted from Fig. 2.5 in [5].

onto an aluminum base which is placed on a large brass block with a thermoelectric cooler (TEC) sandwiched in between. The temperature of the whole assembly on the aluminum base is regulated by a thermoelectric cooler (TEC) controlled by a temperature controller (ILX Lightwave LDT-5525).

For reliable operation of the MOT, the output frequencies of the diode lasers need to be stabilized through feedback loops. Without stabilization, the output frequencies drift (or worse yet, the lasers mode-hop) even with a small disturbance such as a small change in ambient temperature, vibration, noise, and so on. The laser 


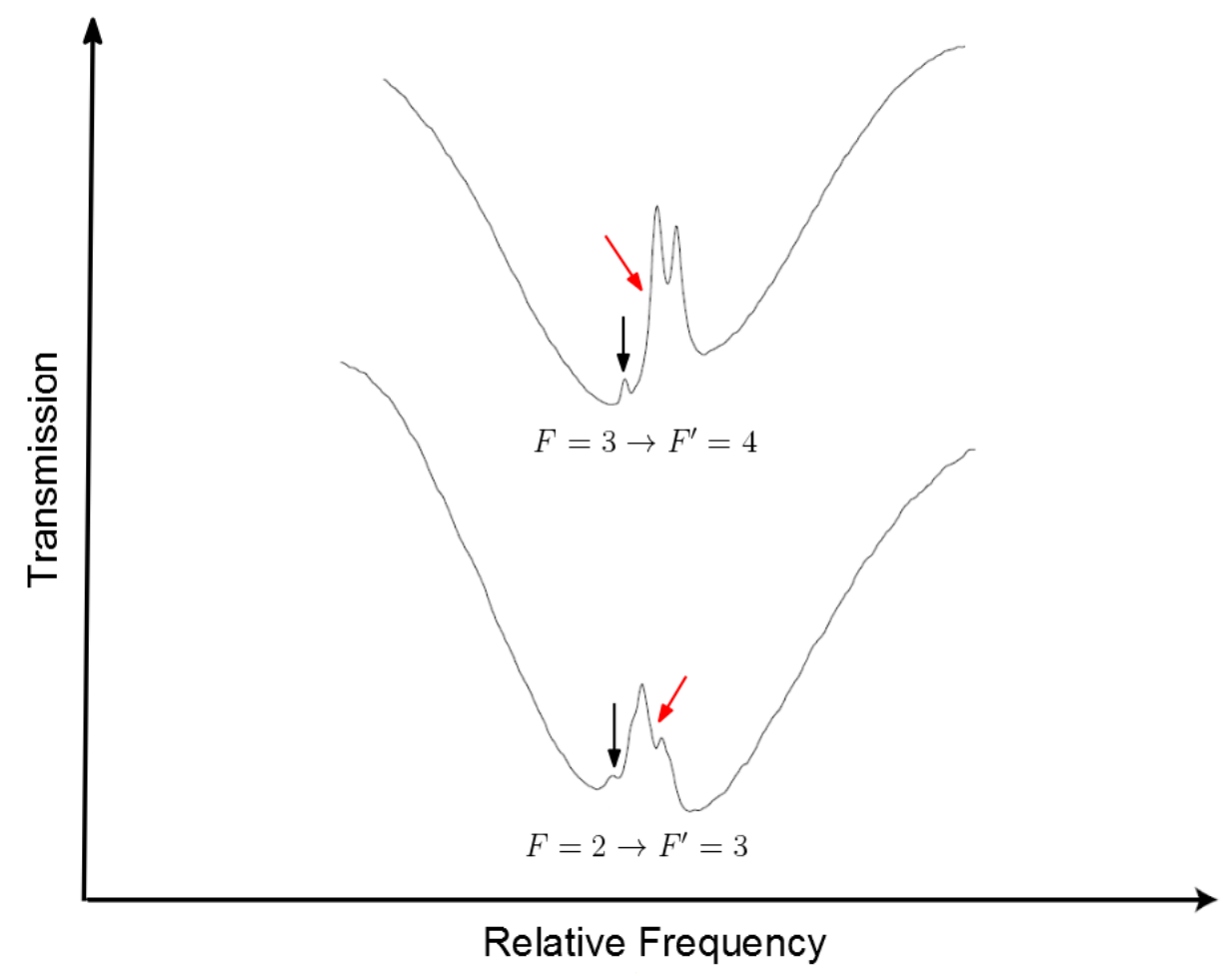

Figure 2.4: The saturated absorption spectroscopy (SAS) signal used to frequency lock the lasers. The top spectrum is for the trap laser and the bottom spectrum is for the repump laser. The black vertical arrows represent the peaks that correspond to the transitions labeled below. The red slanted arrows represent the side-lock point. The figure is adapted from Fig. 2.7 in [5].

frequencies, therefore, are locked using a saturated absorption spectroscopy (SAS) signal. The SAS signal is obtained by directing a small percentage of the laser output to a SAS setup which consists of a Rb vapor cell, a photodiode, and necessary optics. When the laser frequency is scanned by applying a sine wave to the grating-tuning piezo, the saturated absorption spectrum can be seen, as shown by Fig. 2.4. 


\subsubsection{Magnetic Field}

The magnetic field gradient is generated by a pair of anti-Helmholtz coils. The magnetic field generated by the coils is zero at the center of the MOT, and increases approximately linearly in all directions. The expression for the magnetic field and magnetic field gradient can be found on page 17 of [5]. The coils are separated by $12 \mathrm{~cm}$ and each coil is $15 \mathrm{~cm}$ in diameter. The coils are formed with copper magnet wire with Polyurethane-Nylon coating wrapped around a water cooled aluminum coil form. The typical operating current for the coils is about $12 \mathrm{~A}$. From the expression for the magnetic field gradient from [5] and 95 turns of wire for each coil, the magnetic field gradient is found to be 18 Gauss $/ \mathrm{cm}$.

Depending on the type of experiments, the magnetic field is either always on ("non-switching" mode) or turned off during the experiment ("switching" mode). The non-switching mode is suitable when the experiment demands a high density MOT. In other cases, the magnetic field is unwanted because its inhomogeneity causes broadening $(\sim 5 \mathrm{MHz})$ of the resonances. In switching mode, a circuit designed by a former researcher[5] is used to turn off the magnetic field 3 or 4 milliseconds before the excitation laser pulse comes in and turns the field back on $1 \mathrm{~ms}$ after the excitation laser pulse. It is necessary to turn off the magnetic field several ms before because the induced magnetic field, due to Eddy currents, goes away slowly. Direct measurement of the residual magnetic field by Li using a small pick-up coil [5] shows that there is still a residual magnetic field more than $300 \mu$ s after turning off the magnetic field. Park [8] and Levac[4] investigated how the shape of the resonant peak of an atomic transition $n s_{1 / 2} \rightarrow n p_{1 / 2}$ changes with different switch-off delay times. They found 
that the effect of residual magnetic field is highly visible unless the magnetic field is switched off at least $3 \mathrm{~ms}$ before the excitation. With that said, although increasing the switch-off delay does reduce the residual magnetic field, it also results in a lower number of trapped atoms. For that reason, a compromise needs to be made and a switch-off delay of 3 or $4 \mathrm{~ms}$ is used.

\subsubsection{Determining the Trap Parameters}

It is important to know the density of the Rydberg atoms in the MOT because it is directly related to the strength of dipole-dipole interaction, which plays a key role in many of the experiments involving Rydberg atoms in MOT. The appendix of Han's thesis[2] has a detailed description about how various parameters of MOT can be determined. Therefore, only a brief description will be provided. The values given here are measured with the same technique.

The number of atoms in MOT can be determined by measuring the power of the fluorescence. Once the power is known, the total number of atoms in MOT can be calculated by

$$
N=2 \times \frac{P}{E}
$$

where $P$ is the total power of the fluorescence, and $E$ is the power emitted by a single $5 p_{3 / 2}$ atom. The factor of 2 is added because half of the atoms are in $5 p_{3 / 2}$ while the other half are in $5 s_{1 / 2}$ (assuming that the trap laser saturates the $5 s_{1 / 2} \rightarrow 5 p_{3 / 2}$ transition) and only the atoms in $5 p_{3 / 2}$ can emit a a photon. From the measured 
total power of the fluorescence of $4.58 \mu \mathrm{W}$ and the value of $9.4 \times 10^{-12}$ for $E$, we get

$$
N=4.87 \times 10^{5} \quad(\text { Total Number of Trapped Atoms) }
$$

The size of the trap can be measured with a linear CCD array connected to an oscilloscope. The linear CCD array has a pixel size of $0.014 \mathrm{~mm}$, which corresponds to $5 \mu$ s on the oscilloscope. The density distribution of the trapped atoms is assumed to be Gaussian. Fig. 2.5 shows a Gaussian fit of the image of trapped atoms. From the full width at half maximum (FWHM) of $0.22 \mathrm{~ms}$, the conversion factor given above, and the magnification introduced by the focusing lens (which is 1 in this case because the lens is placed exactly midway between the linear CCD array and the trapped atoms), the size of the trap (FWHM) is determined to be $0.62 \mathrm{~mm}$.

$$
\text { Size of the Trap }(\mathrm{FWHM})=0.62 \mathrm{~mm}
$$

Once the total number of trapped atoms and the size of the trap are known, it is straightforward to calculate the density. It is given by

$$
\frac{N}{\frac{4}{3} \pi w_{T}^{3}}=\frac{4.87 \times 10^{5}}{\frac{4}{3} \pi\left(\frac{0.616 \mathrm{~mm}}{2}\right)^{3}}=8.2 \times 10^{9} \mathrm{~cm}^{-3} \quad \text { (Trap Avg. Density) }
$$

where $w_{T}$ is the radius of the MOT.

The risetime of the trap can be measured by recording the fluorescence of the trap as a function of time with a photodiode when one of the lasers is suddenly unblocked. The risetime of the trap represents how quickly the trap can be replenished and is 


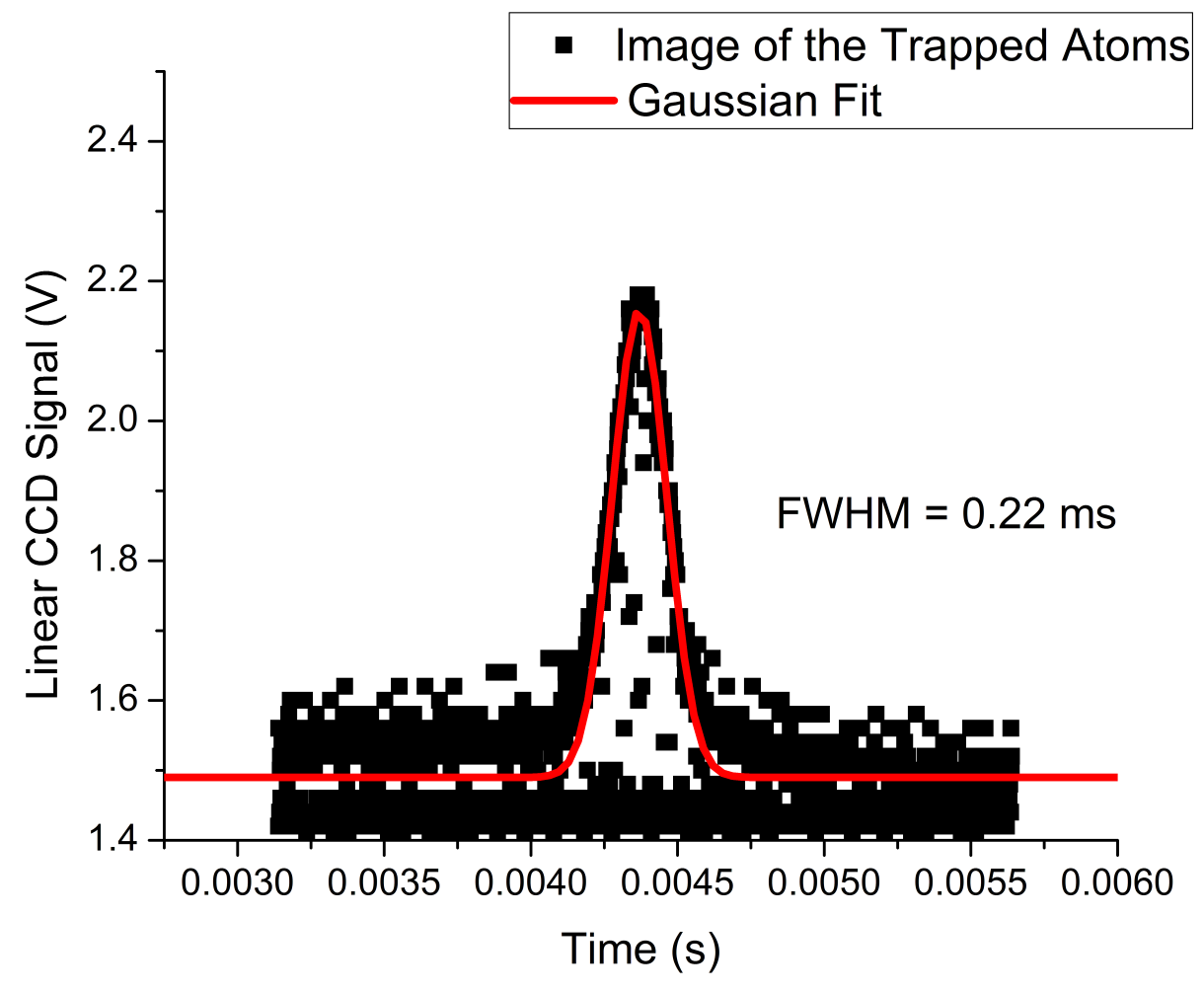

Figure 2.5: The image of trapped atoms and a Gaussian fit. 
largely dependent on the number of Rubidium atoms present in the vacuum chamber. In turn, the getter current mostly determines the risetime. The recorded fluorescence vs. time data is fitted with an expression of the form $N(t)=N_{\max }\left(1-e^{\frac{t}{\tau}}\right)$ to extract the value for $\tau$. The risetime was measured to be between 1 and $2 \mathrm{~s}$ depending on the getter current.

The number of Rydberg atoms can be determined from the following equation:

$$
N_{\text {Rydberg }}=\left(N_{\text {max }}-N_{\text {avg }}\right) R_{\text {load }} \Delta t
$$

where $N_{\max }$ is the number of trapped atoms, $N_{\text {avg }}$ is the number of trapped atoms with $480 \mathrm{~nm}$ excitation laser, $R_{\text {load }}=\frac{1}{\tau}$ is the loading rate which is the inverse of the risetime, and $\Delta t$ is the time interval between two adjacent laser shots. Using $N_{\text {max }}=4.87 \times 10^{5}, N_{\text {avg }}=0.45 \times N_{\text {max }}, R_{\text {load }}=\frac{1}{1.5 \mathrm{~s}}$, and $\Delta t=0.05 \mathrm{~s}$,

$$
N_{\text {Rydberg }}=8.9 \times 10^{3} \quad \text { (Number of Rydberg Atoms) }
$$

Once the number of Rydberg atoms is known, the density of Rydberg atoms at trap center can be determined from the following equation:

$$
\rho_{0}=\frac{N}{\sqrt{\pi^{3}} w_{T} w_{L}^{2}}
$$

where $w_{T}$ is the radius of the MOT and $w_{L}$ is the radius of the $480 \mathrm{~nm}$ laser beam. $w_{L}$ can be measured with knife edge method and is found to be $197 \mu \mathrm{m}$. Therefore, the density of Rydberg atoms at trap center is 


$$
\left.\rho_{0}=\frac{8.9 \times 10^{3}}{\sqrt{\pi^{3}}(0.305 \mathrm{~mm})(98.5 \mu \mathrm{m})}=5.40 \times 10^{8} \mathrm{~cm}^{-3} \quad \text { (Density of Rydberg Atoms }\right)
$$

The Rydberg atom density as a function of the position has the following form: $\rho(x, y, z)=\rho_{0} e^{-\left(x^{2}+y^{2}+z^{2}\right) / w_{T}^{2}} e^{-\left(x^{2}+y^{2}\right) / w_{L}^{2}}$, where $\mathrm{x}, \mathrm{y}$, and $\mathrm{z}$ are the Cartesian displacements from the center of the trap. The $480 \mathrm{~nm}$ beam propagates in the $\mathrm{z}$ direction.

The density of Rydberg Atoms is related to the average spacing between the Rydberg atoms by the following expression:

$$
R_{a v}=\left(\frac{3}{4 \pi \rho}\right)^{1 / 3}
$$

A density of $5.40 \times 10^{8}$ leads to the average spacing of $7.6 \mu \mathrm{m}$.

Another useful expression is the most probable nearest neighbor separation assuming random distribution and is given by:

$$
R_{n n}=\frac{1}{(2 \pi \rho)^{1 / 3}}
$$

$R_{a v}$ and $R_{n n}$ are related by

$$
R_{n n}=0.874 \times R_{a v}
$$




\subsection{Excitation of Rydberg Atoms}

The trapped atoms in the $5 p_{3 / 2}$ state are excited to Rydberg states by a laser pulse with a wavelength near $480 \mathrm{~nm}$. The laser pulse is generated by pulse amplifying, at a $20 \mathrm{~Hz}$ repetition rate, and then frequency doubling the output of a tapered amplifier seeded by a $960 \mathrm{~nm}$ single mode diode laser. The resulting pulse has an energy of $10 \mu \mathrm{J}$, is $10 \mathrm{~ns}$ long, and has a bandwidth of $150 \mathrm{MHz}$. In certain situations, a dye laser can be used instead when a quick tuning of the wavelength is required.

\subsubsection{Dye Amplified Diode Laser}

The Toptica DL100 external-cavity diode laser with an output wavelength centered at $960 \mathrm{~nm}$ provides the seed beam. The coarse tuning of the wavelength can be achieved by slowly turning the screw that changes the diffraction grating angle. After the coarse tuning, fine tuning is done by changing current, temperature, and the DC voltage applied to the piezo that changes the diffraction grating angle. To get a stable Rydberg signal, the output has to be single-mode and be free from mode hops near the resonance. The laser has a typical output power of $50 \mathrm{~mW}$. The output beam is sent through two isolators which are used to prevent any reflected beam from entering the laser. The output is then amplified with a tapered amplifier, which is capable of amplifying the power upto $0.5 \mathrm{~W}$. A portion of the beam is directed to a Fabry-Perot cavity (Coherent Spectrum Analyzer) to check that the laser is operating with single mode and to monitor any shift in output frequency. The beam is further amplified by two dye cells pumped by the second harmonic of a Nd:YAG laser (Quanta-Ray 


\begin{tabular}{ccccc} 
Wavelength & Dye & Pumped with & Concentration & Solvent \\
\hline $960 \mathrm{~nm}$ & LDS 965 & $532 \mathrm{~nm}$ & $0.12 \mathrm{~g} / \mathrm{L}$ & $\begin{array}{c}0.85 \text { Propylene Carbonate } \\
0.15 \text { Ethylene Glycol }\end{array}$ \\
\hline $480 \mathrm{~nm}$ & Coumarin 480 & $355 \mathrm{~nm}$ & $0.4 \mathrm{~g} / \mathrm{L}$ & 1.00 Methanol
\end{tabular}

Table 2.1: The list of dyes used for dye amplification stages

DCR-2A), and focused onto a Potassium Niobate $\left(\mathrm{KNbO}_{3}\right)$ crystal to double the frequency. The frequency doubled $480 \mathrm{~nm}$ laser beam is sent through one more dye amplification stage which is pumped by the third harmonic of a Nd:YAG laser before it is directed toward the vacuum chamber. The list of the dyes used for dye amplification stages is given in Table 2.1. The beam enters the vacuum chamber from the top.

\subsubsection{Dye Laser}

Although the dye amplified diode laser is the preferred method to excite Rydberg atoms, it is sometimes beneficial to use a dye laser instead. Dye lasers use organic molecules as the active medium. Depending on the desired output frequency and the pumping source (which is typically a harmonic of Nd:YAG laser), different dyes need to be used. Since the excitation of Rb atoms from $5 p_{3 / 2}$ to Rydberg states is in the vicinity of $480 \mathrm{~nm}$, we use Coumarin 480 pumped by the third harmonic (355 $\mathrm{nm}$ ) of the Nd:YAG laser.

The main selling point of the dye laser is its easy tunability. In order to change the wavelength of the diode laser, it is typically necessary to change three parameters: current, temperature, and the grating angle. On the other hand, it is much more straightforward to change the wavelength of a dye laser, as it usually only requires 
the grating angle to be changed slightly. The trade off is the fact that the dye laser is inherently multi-mode, with a linewidth in excess of $10 \mathrm{GHz}$, and fine tuning of the wavelength is impossible.

We use a dye laser with the Littman configuration that is constructed from homebuilt aluminum mounts, UV cell assembly, and a diffraction grating. An end mirror, a diffraction grating mounted at grazing incidence, and a tuning mirror form a cavity. The zeroth order reflection from the grating is the output of the laser, while the first order reflection is sent to the tuning mirror which reflects it and sends it back to the cavity. The tuning of the wavelength is achieved by turning the tuning mirror.

\subsection{Microwave Setup}

Since the energy differences between neighboring states of atoms excited to Rydberg states often fall in the microwave frequency range $(0.3-300 \mathrm{GHz})$, it makes using microwaves an attractive choice to probe transitions between Rydberg states. Through the use of various frequency multipliers, we are able to generate microwaves with frequencies up to $110 \mathrm{GHz}$. The microwaves are generated in an Agilent 83622B (or Agilent E8247E) synthesizer, which has a maximum frequency of $20 \mathrm{GHz}$, and a General Microwave DM862B switch is used to form the microwaves into pulses. A typical microwave pulse length is $1 \mu \mathrm{s}$. Depending on the desired output frequency range, different frequency multipliers are used. Table 2.2 shows the list of frequency multipliers we have as well as their input and output frequency ranges. The relative microwave power is controlled in the final waveguide with a precision attenuator for 


\begin{tabular}{cccc} 
Model Number & Type & $\begin{array}{c}\text { Input Range } \\
(\mathrm{GHz})\end{array}$ & $\begin{array}{c}\text { Output Range } \\
(\mathrm{GHz})\end{array}$ \\
\hline HP 83554A & passive doubler & $13.25-20$ & $26.5-40$ \\
Narda DBS2640X220 & active doubler & $13.25-20$ & $26.5-40$ \\
Narda DBS4060X410 & active quadrupler & $10-15$ & $40-60$ \\
HP 83556A & passive tripler & $13.3-20$ & $40-60$ \\
Pacific Millimeter V2 & passive doubler & $25-37.5$ & $50-75$ \\
Pacific Millimeter W3WO & passive tripler & $25-36.67$ & $75-110$
\end{tabular}

Table 2.2: The list of microwave frequency multipliers used throughout the experiments in this dissertation. Pacific Millimeter V2 and W3WO need to be used in conjunction with an active doubler (Narda DBS 2640X220).

the frequency range being used. The microwaves are emitted from a horn outside the vacuum chamber and propagate through a window to the trapped sample of atoms. The vertical rods that are used to apply field ionization pulse can scatter the microwaves to some degree, and this can result in the polarization's not being perfectly linear. Additional information about the microwaves scattering from the rods may be found in Chapter 4 of Levac's thesis[4].

The active frequency multipliers are capable of producing higher power than passive frequency multipliers. Both the active doubler and quadrupler have a typical output power of $+20 \mathrm{dBm}$ given an input power of $+15 \mathrm{dBm}$. As a comparison, the HP 83554A passive doubler and the HP 83556A passive tripler have maximum leveled output power of $+8 \mathrm{dBm}$ and $+3 \mathrm{dBm}$, respectively. Although it is difficult to measure the output power from the horn accurately, several methods are employed in order to estimate the microwave field amplitude at the trap center. The first method is computing the field directly by using the microwave power right before the horn, the known gain of the horn antenna, and the estimated distance from 
the horn to the trap center. The second method is relating the full width at half maximum of a resonant peak of an atomic transition to the Rabi frequency. Lastly, the third method is using the AC Stark shift of a transition and computing how much field is required to produce the observed shift. All three methods produce a reasonably consistent value. For example, the maximum microwave field amplitude at trap center that can be produced by Narda DBS4060X410 active quadrupler is found to be approximately $0.7 \mathrm{~V} / \mathrm{cm}$.

\subsection{Detection and Data Acquisition}

\subsubsection{Field Ionization}

We use state-selective field ionization and a microchannel plate (MCP) detector to detect Rydberg atoms. A home-made circuit generates a high voltage pulse which has a typical risetime of $3.5 \mu \mathrm{s}$ and a maximum peak amplitude in excess of $4 \mathrm{kV}$. The trapped atoms are located at the center of four rods as shown by Fig. 2.2, and the pulse is applied to the pair of rods farthest from the MCP detector. The pulse ionizes the Rydberg atoms and drives the resulting ions or electrons toward the MCP detector. The circuit generates the pulse by first storing electrical energy in a capacitor then releasing it into the primary coil of a trigger transformer. A silicon controlled rectifier (SCR) is used as a switch and releases the stored charge at a desired time. Fig. 2.6 shows the circuit diagram. The diagram shows only one of many possible designs for the circuit. Different components and/or design can be adopted to meet specific requirements. 


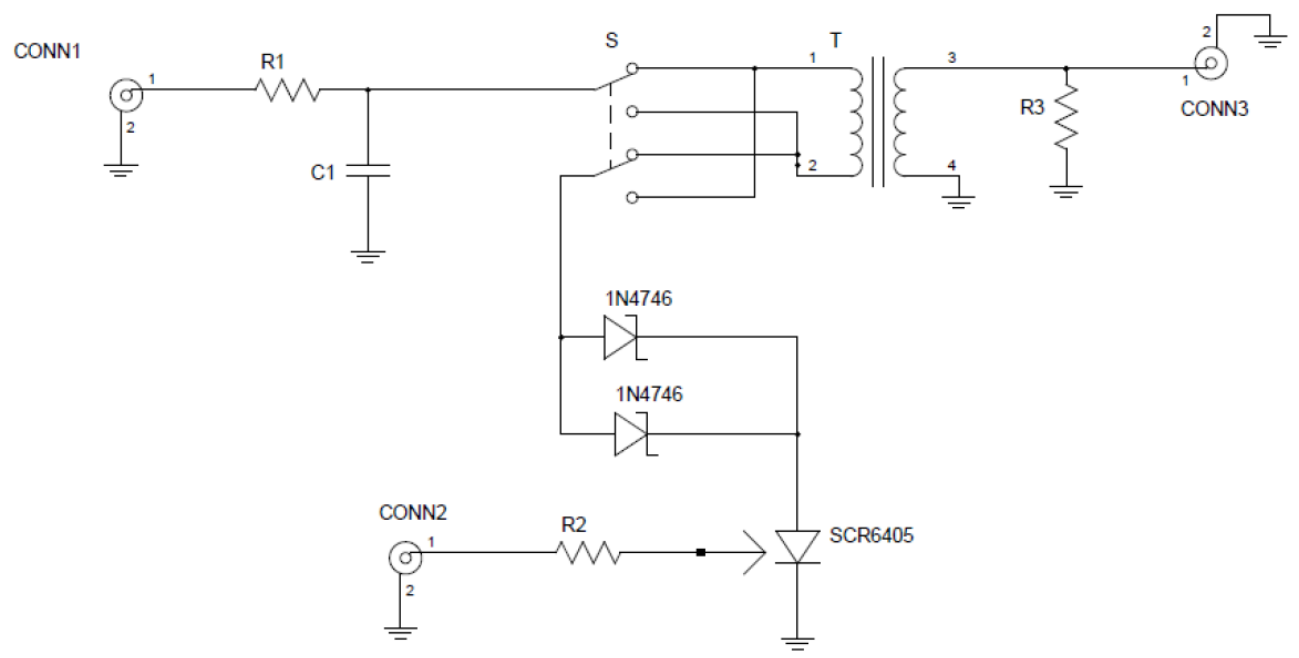

Figure 2.6: Field ionization circuit diagram. It is taken from Fig. 2.17 of [4]. The parameters are R1: $8 \Omega, \mathrm{R} 2: 51.8 \Omega, \mathrm{R} 3: 100 \mathrm{k} \Omega$ (Optional), and C1: $47 \mu \mathrm{F}$. CONN1, CONN2, and CONN3 are DC Voltage, trigger pulse, and output, respectively. The trigger transformer has a turns-ratio of 30 .

\section{Slow and Fast Field Ionization Pulse}

The typical risetime of the circuit shown in Fig. 2.6 is $3.5 \mu \mathrm{s}$. Although this provides excellent state-selectivity, a field ionization pulse with a shorter risetime is useful at times. For instance, Han performed an experiment in which she compared the ionization signal from slow rising and fast rising pulses and reported that if the initial rise of the field ionization pulse is slow, the passage through the molecular avoided crossings can be adiabatic[3]. The fast field ionization pulse can be generated by connecting the output of a pulse generator that is capable of generating high voltage pulse directly to the primary coil of a trigger transformer. By using a HP 214B pulse generator, which is capable of generating $100 \mathrm{~V}$ square pulses, in conjunction with 30 turns-ratio trigger transformer, we are able to get a field ionization pulse with a 
risetime of $1 \mu \mathrm{s}$ and a maximum peak amplitude of around $500 \mathrm{~V}$. The limiting factor is the maximum amplitude of the square pulse input. In practice, the maximum peak amplitude of $500 \mathrm{~V}$ means that we cannot ionize states with $n^{*}<38$. In order to ionize the $n^{*}<38$ states using a fast field ionization pulse, a more complicated scheme is needed. In contrast, the slow pulse can reach a peak amplitude in excess of $4 \mathrm{kV}$. The lowest state that can be ionized with the current setup $22 d$. However, arcs start to appear inside the vacuum chamber at this voltage which limits us from increasing the voltage further.

\subsubsection{Ion Detection and Electron Detection}

The experimental setup is capable of detecting either ions or electrons. A minor modification is necessary to change from ion detection to electron detection. The steps are given on page 31 of Levac's thesis [4]. Since electrons are much lighter than ions, electrons reach the detector almost instantaneously after ionization whereas ions take several microseconds. A noteworthy thing about ion detection is that it becomes increasingly more difficult to distinguish between the signals from different states with high $n^{*}$. Figs. 2.8 and 2.9 show the times at which the ionization signal appears as the excitation laser tuning with respect to the ionization limit of Rubidium is changed. They clearly show that we lose state-selectivity with ion detection at laser tuning of $-1750 \mathrm{GHz}$ and above. Increasing the risetime by reducing the DC voltage input can alleviate this problem to some degree, yet it is important to be aware of this limitation with ion detection. 


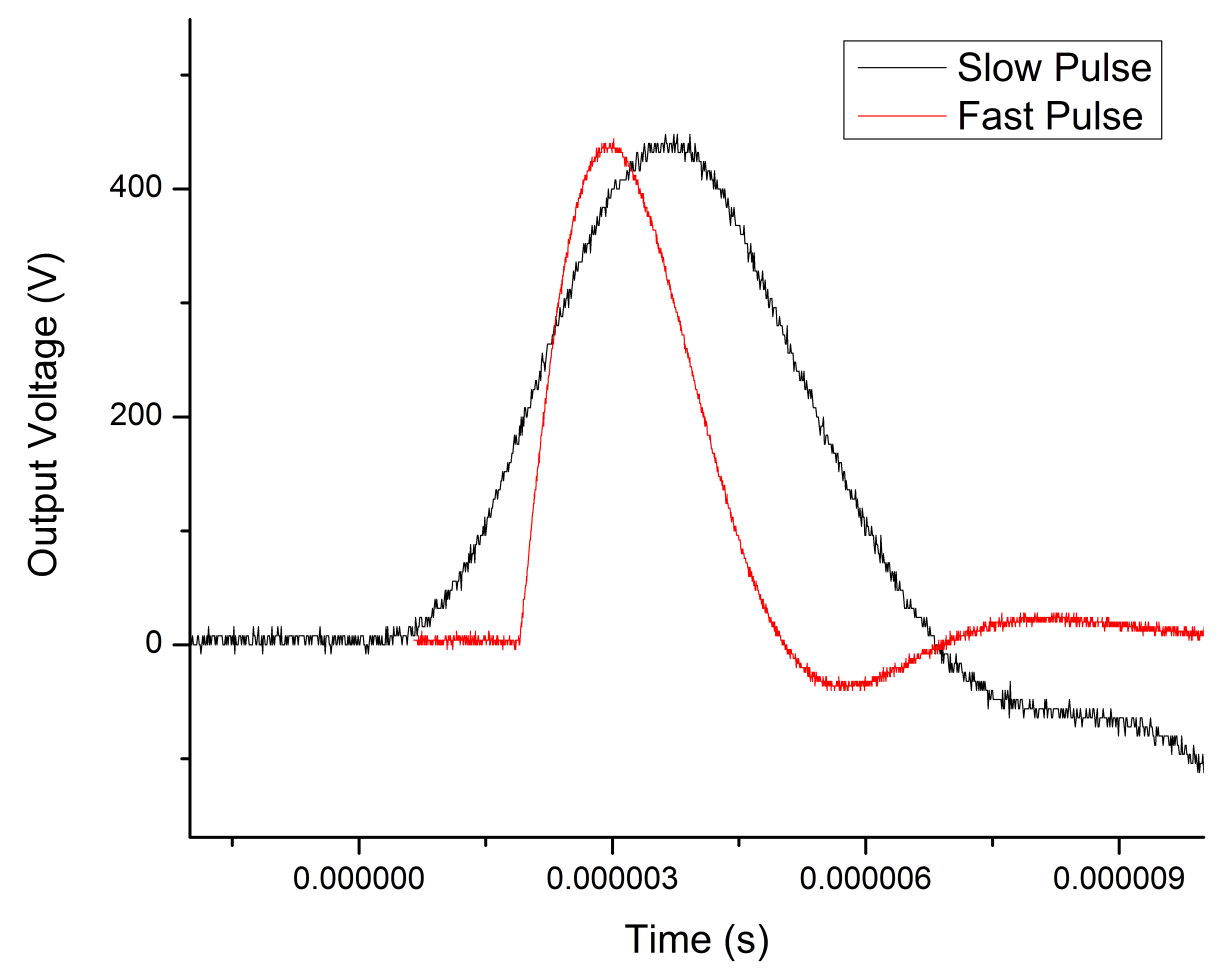

Figure 2.7: Fast and slow field ionization pulses. The fast pulse has a risetime of 1 $\mu \mathrm{s}$ and the slow pulse has a risetime of $3.5 \mu \mathrm{s}$. 


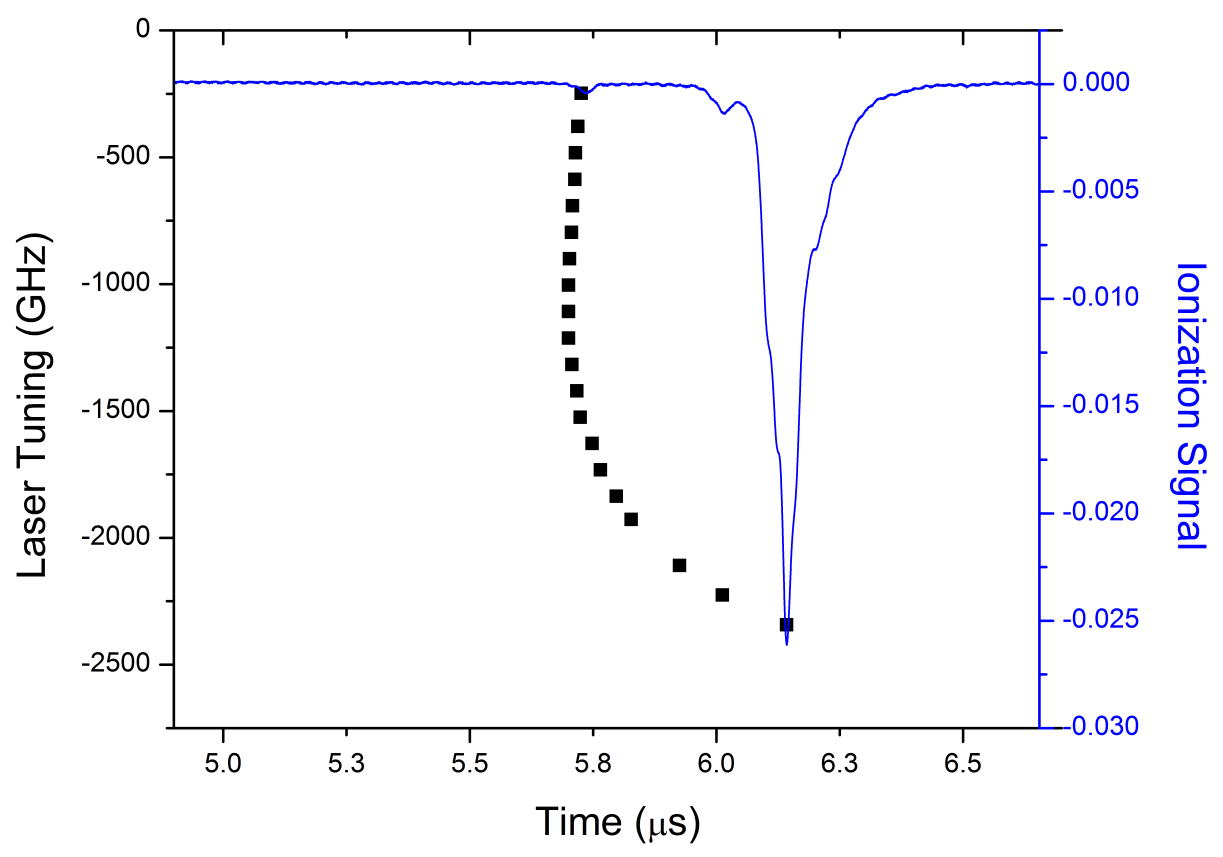

Figure 2.8: This figure shows the times at which the ionization signal appears as the excitation laser tuning with respect to the ionization limit of Rubidium is changed when ions are being detected. The slow field ionization pulse with a peak amplitude of $500 \mathrm{~V}$ was used to obtain this data. It is clear that at laser tuning of $-1750 \mathrm{GHz}$ and above, the ionization signals always appear at $5.7 \mu \mathrm{s}$. This shows that we lose state-selectivity of the states with high $n^{*}$. 


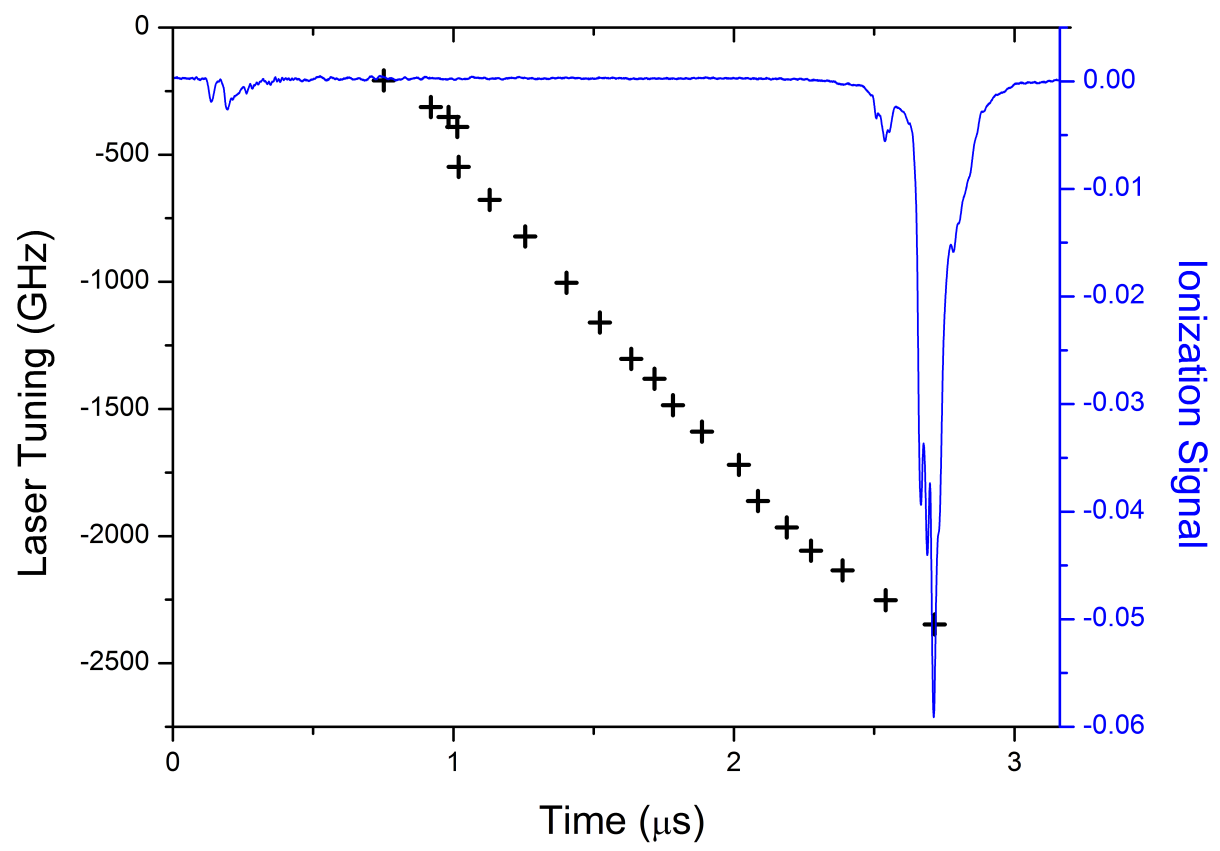

Figure 2.9: This figure shows the times at which the ionization signal appears as the excitation laser tuning with respect to the ionization limit of Rubidium is changed when electrons are being detected. The slow field ionization pulse with a peak amplitude of $500 \mathrm{~V}$ was used to obtain this data. Different from ion detection, there is no loss of state-selectivity.

\subsection{The Timing Diagram}

Fig. 2.10 shows a timing diagram for a typical experiment. Since the $480 \mathrm{~nm}$ pulse, which produces Rydberg atoms, has a $20 \mathrm{~Hz}$ repetition rate, the experiment is repeated at the same rate. A Stanford Research Systems DG535 digital delay generator, which is triggered by the Q-Switch pulse from the Nd:YAG laser, is used to control the timings of the magnetic field switching, microwave pulse, and the field 
ionization pulse. As mentioned previously, the magnetic field is switched off 3 or 4 ms before the $480 \mathrm{~nm}$ pulse when we use the "switching" mode.

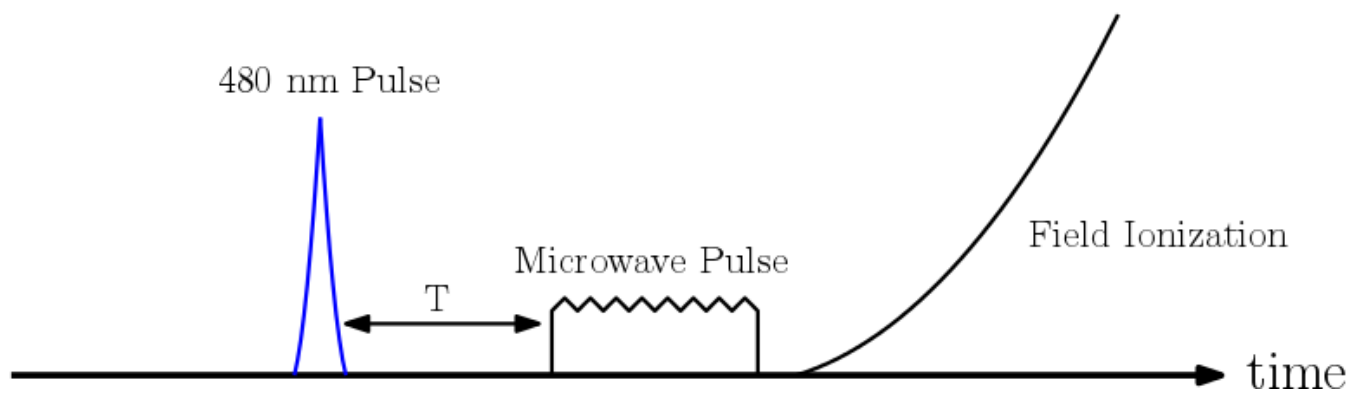

Figure 2.10: The timing diagram for a typical experiment. $T$ is the delay between the Rydberg excitation and the beginning of the microwave pulse. Typically, the microwave pulse is sent in immediately following the $480 \mathrm{~nm}$ excitation. The microwave pulse typically has a width that is on the order of $1 \mu \mathrm{s}$. The whole sequence repeats at $20 \mathrm{~Hz}$ frequency, which is the repetition rate of the $480 \mathrm{~nm}$ pulse. 


\section{REFERENCES}

[1] Anderson, W. R. (1996). Resonant Dipole-Dipole Collisions of Rydberg Atoms in a Magneto-Optical Trap. PhD thesis, University of Virginia.

[2] Han, J. (2009). Dipole effects in a cold Rydberg gas. PhD thesis, University of Virginia.

[3] Han, J. \& Gallagher, T. F. (2008). Field ionization of the frozen rydberg gas. Phys. Rev. A, 77, 015404.

[4] Levac, L. (2013). Observation of the dipole-dipole interaction in dressed state rydberg atoms by microwave spectroscopy. Master's thesis, University of Virginia.

[5] Li, W. (2005). Probing Dipole-Dipole Interactions in a Frozen Rydberg Gas with Millimeter Waves. PhD thesis, University of Virginia.

[6] MacAdam, K. B., Steinbach, A., \& Wieman, C. (1992). A narrow-band tunable diode laser system with grating feedback, and a saturated absorption spectrometer for Cs and Rb. American Journal of Physics, 60, 1098-1111.

[7] Metcalf, H. J. \& van der Straten, P. (2003). Laser cooling and trapping of atoms. J. Opt. Soc. Am. B, 20(5), 887-908.

[8] Park, H. (2012). Observation of the dipole-dipole interaction between cold Rydberg atoms by microwave spectroscopy. PhD thesis, University of Virginia.

[9] Pritchard, J. (2011). Cooperative Optical Non-linearity in a blockaded Rydberg Ensemble. PhD thesis, Durham University.

[10] Wieman, C. E. \& Hollberg, L. (1991). Using diode lasers for atomic physics. Review of Scientific Instruments, 62(1), 1-20. 


\section{Chapter 3}

\section{Molecular Transition}

\subsection{Introduction}

There is substantial interest in molecules composed of one or more Rydberg atoms.

The most well studied are the trilobite molecules in which a ground state atom or molecule is bound to a Rydberg atom by its short range interaction with the Rydberg electron $[2,8,20]$. Less well studied are molecules composed of more than one Rydberg atom. The first proposed were macrodimers based on the long range van der Waals interactions of two Rydberg atoms in the same state [3, 18]. Later proposals for even longer range molecules were based on dipole-dipole interactions of atoms in different states $[12,13]$. While no experimental evidence for stable bound double Rydberg molecules has been presented, there is evidence for the existence of transient macrodimers. In laser excitation spectra, signatures of transient molecules formed by dipole-dipole, van der Waals, and dipole-quadrupole interactions have 
been observed $[4,7,16]$. Forster resonant energy transfer involving two, three, and even four atoms has been observed $[1,6,9,15]$. Finally, microwave transitions involving pairs of Rydberg atoms have been observed [24].

Here we report a systematic study of microwave transitions in which a pair of atoms absorbs a microwave photon, with the result that both atoms change state. Specifically, we have examined the process

$$
n d_{5 / 2} n d_{5 / 2} \rightarrow(n+1) d_{j}(n-2) f_{7 / 2} .
$$

Here $n$ is the principal quantum number, and the molecular states are labelled by the atomic states of the two atoms. The process of Eq. (3.1) is observable due to the dipole-dipole configuration interaction (CI) between the $n d_{5 / 2} n d_{5 / 2}$ state and the energetically nearby $(n+2) p_{3 / 2}(n-2) f_{7 / 2}$ state.

$$
n d_{5 / 2} n d_{5 / 2} \leftrightarrow(n+2) p_{3 / 2}(n-2) f_{7 / 2}
$$

For completeness, we have also verified that the molecular transition

$$
n d_{5 / 2} n d_{5 / 2} \rightarrow(n+2) p_{3 / 2}(n-1) d_{5 / 2}
$$

occurs, due to the same CI. The CI enabled microwave transition is roughly analogous to a two photon transition, with the $(n+2) p_{3 / 2}(n-2) f_{7 / 2}$ state playing the role of the off resonant intermediate state, and the dipole-dipole interaction of Eq. (3.2) playing the role of one of the photons. 


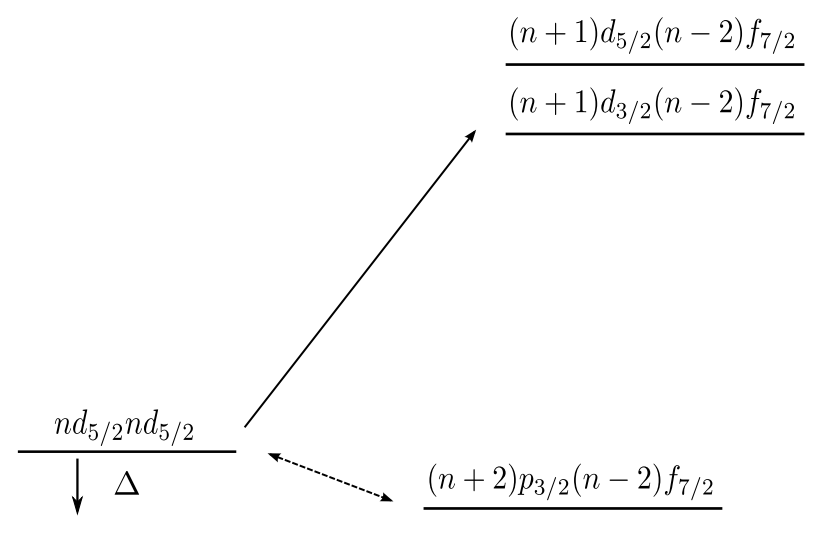

Figure 3.1: Energy levels for the $n d_{5 / 2} n d_{5 / 2} \rightarrow(n+1) d_{j}(n-2) f_{7 / 2}$ microwave transition, shown by the solid arrow. This transition is allowed due to the configuration interaction between the $n d_{5 / 2} n d_{5 / 2}$ and $(n+2) p_{3 / 2}(n-2) f_{7 / 2}$ state, shown by the broken double headed arrow.

Systematic measurements of the microwave transition of Eq. (3.1) over the range $35 \leq n \leq 42$ show that the transition probability for the process of Eq. (3.1) can be described by a simple CI matrix element, in spite of the fact that over this range of $n$ the microwave power required to drive the transition changes by more than three orders of magnitude. In the sections which follow we present a model for the transition of Eq. (3.1), describe our experimental procedure, and present our results.

\subsection{Theory - Configuration Interaction Model}

The relevant energy levels for the microwave transition $n d_{5 / 2} n d_{5 / 2} \rightarrow(n+1) d_{j}(n-$ 2) $f_{7 / 2}$ are shown in Fig. 3.1. All other levels can be ignored. This transition is 


\begin{tabular}{cc}
$n$ & $\Delta(\mathrm{GHz})$ \\
\hline 34 & 1.8798 \\
35 & 1.4820 \\
36 & 1.1548 \\
37 & 0.8849 \\
38 & 0.6621 \\
39 & 0.4778 \\
40 & 0.3253 \\
41 & 0.1991 \\
42 & 0.09465 \\
44 & -0.06288
\end{tabular}

Table 3.1: Energy detunings $(\Delta)$ between $n d_{5 / 2} n d_{5 / 2}$ and $(n+2) p_{3 / 2}(n-2) f_{7 / 2}$

allowed because the $n d_{5 / 2} n d_{5 / 2}$ state is coupled to the nearby $(n+2) p_{3 / 2}(n-2) f_{7 / 2}$ state by the dipole-dipole interaction, shown by the double headed broken arrow in Fig. 3.1, and some of the $(n+2) p_{3 / 2}(n-2) f_{7 / 2}$ state is admixed into the $n d_{5 / 2} n d_{5 / 2}$ state. The dipole matrix element connecting the $n d_{5 / 2}$ state to the $(n-2) f_{5 / 2}$ state is a factor of four smaller than the one connecting it to the $(n-2) f_{7 / 2}$ state [5]. For this reason we see no evidence for transitions to final states containing an $(n-2) f_{5 / 2}$ state. At finite internuclear separation $R$, the $n d_{5 / 2} n d_{5 / 2}$ state can be written as

$$
\left|n d_{5 / 2} n d_{5 / 2}\right\rangle_{R}=\left|n d_{5 / 2} n d_{5 / 2}\right\rangle+\epsilon\left|(n+2) p_{3 / 2}(n-2) f_{7 / 2}\right\rangle,
$$

where the molecular states without the subscripts are the $R=\infty$ states and the admixture coefficient $\epsilon$ is given by

$$
\begin{aligned}
\epsilon & =\frac{\left\langle n d_{5 / 2} n d_{5 / 2}\left|\mu \mu^{\prime}\right|(n+2) p_{3 / 2}(n-2) f_{7 / 2}\right\rangle}{R^{3} \Delta} \\
& =\frac{\left\langle n d_{5 / 2}|\mu|(n+2) p_{3 / 2}\right\rangle\left\langle n d_{5 / 2}\left|\mu^{\prime}\right|(n-2) f_{7 / 2}\right\rangle}{R^{3} \Delta} .
\end{aligned}
$$


Here $\mu$ and $\mu^{\prime}$ are the dipole moments of atoms 1 and 2. We ignore exchange effects. The admixture coefficient is the ratio of the dipole-dipole coupling to the energy detuning $\Delta$ between the $n d_{5 / 2} n d_{5 / 2}$ and $(n+2) p_{3 / 2}(n-2) f_{7 / 2}$ states at $R=\infty$, as shown in Fig. 3.1. Explicitly, the detuning is given by

$$
\Delta=W_{n d_{5 / 2} n d_{5 / 2}}-W_{(n+2) p_{3 / 2}(n-2) f_{7 / 2}} .
$$

The detunings between the $n d_{5 / 2} n d_{5 / 2}$ and $(n+2) p_{3 / 2}(n-2) f_{7 / 2}$ states are given in Table 3.1. The energies of the atomic states have been calculated using the quantum defects given by Refs. $[11,14]$. The $n d_{5 / 2} n d_{5 / 2}-(n+1) d_{j}(n-2) f_{7 / 2}$ microwave transition matrix element is

$$
\begin{aligned}
\left\langle\left. n d_{5 / 2} n d_{5 / 2}\right|_{R} \mu E\right| & \left.(n+1) d_{j}(n-2) f_{7 / 2}\right\rangle \\
& =\epsilon E\left\langle(n+2) p_{3 / 2}|\mu|(n+1) d_{j}\right\rangle\left\langle(n-2) f_{7 / 2} \mid(n-2) f_{7 / 2}\right\rangle,
\end{aligned}
$$

where $E$ is the microwave field amplitude. In this microwave transition, the $(n-$ 2) $f_{7 / 2}$ atom is a spectator. Since all the dipole matrix elements are approximately proportional to $n^{2}$, the microwave transition matrix element is given by

$$
\left\langle\left. n d_{5 / 2} n d_{5 / 2}\right|_{R} \mu E \mid(n+1) d_{j}(n-2) f_{7 / 2}\right\rangle=\frac{\beta n^{6} E}{\Delta R^{3}}
$$

where $\beta$ is a numerical constant of order one.

To compute the fractional population transfer (FPT) we follow an approach used by Pillet et al. [17]. Consider for a moment two $n d_{5 / 2}$ atoms separated by $R$. They 
are coupled to the $(n+1) d_{j}(n-2) f_{7 / 2}$ state by the coupling matrix element of Eq. (3.8), and a pair initially excited to the $n d_{5 / 2} n d_{5 / 2}$ state oscillates between it and the $(n+1) d_{j}(n-2) f_{7 / 2}$ state at the Rabi frequency $\Omega$, given by

$$
\Omega=\frac{\beta n^{6} E}{|\Delta| R^{3}} .
$$

If the microwave field is present for a time $T$, the $n d_{5 / 2} n d_{5 / 2}$ pair makes half a Rabi oscillation and is left in the $(n+1) d_{j}(n-2) f_{7 / 2}$ state if $\Omega T=\pi$, which occurs for $R=R_{T}$, where $R_{T}$ is defined by

$$
\Omega T=\frac{\beta n^{6} E T}{|\Delta| R_{T}^{3}}=\pi .
$$

If $R<R_{T}$ the pair oscillates more rapidly, and on average the probability of making the transition is $1 / 2$. On the other hand, if $R>R_{T}$, the probability of making the transition drops very rapidly due to the $1 / R^{3}$ dependence of the coupling matrix element of Eq. (3.8). In sum, only pairs with $R<R_{T}$ undergo the transition. Assuming the fraction of pairs making the transition is small, the fractional population transfer (FPT) from the $n d_{5 / 2} n d_{5 / 2}$ state to the $(n+1) d_{j}(n-2) f_{7 / 2}$ state is given by

$$
F P T=\frac{R_{T}^{3}}{R_{a v}^{3}},
$$

where $R_{a v}$ is the average spacing of the pairs, which is related to the density $\rho$ by

$$
\rho=\frac{3}{4 \pi R_{a v}^{3}} .
$$


We implicitly assume that $R_{a v} \gg R_{\text {blockade }}$, where $R_{\text {blockade }}$ is the radius of the blockade sphere [22]. This condition is easily met for our Rydberg atom density . Finally,

$$
F P T=\frac{\beta^{\prime} n^{6} E \rho}{|\Delta|},
$$

where $\beta^{\prime}=4 \beta T / 3$. The fractional population transfer is bilinear in the density of the Rydberg atoms and the microwave field amplitude. As $n$ is changed from 35 to $42, n^{6} / \Delta$ changes by a factor of 50 . Therefore, at a constant density $\rho$ the microwave power needed to observe the same fractional population transfer should change by a factor of 2500 .

While it is straightforward to make relative microwave field or power measurements at a fixed frequency, comparing the microwave fields over the broad frequency range we have used presents more of a problem since we do not know how efficiently the microwave power is transmitted to the interaction region from the horn. The AC Stark shift of the observed resonances, analogous to that observed in two photon spectroscopy, provides a way to calibrate absolutely the microwave field. The $n d_{5 / 2} n d_{5 / 2} \rightarrow(n+1) d_{5 / 2}(n-2) f_{7 / 2}$ transition is nearly resonant with the atomic $(n+2) p_{3 / 2} \rightarrow(n+1) d_{j}$ transition, and the AC Stark shift of these two atomic levels shifts the $(n+1) d_{j}(n-2) f_{7 / 2}$ and $(n+2) p_{3 / 2}(n-2) f_{7 / 2}$ states in opposite directions, as shown in Fig. 3.2, which is drawn for $\Delta>0$.

For a linearly polarized microwave field the azimuthal angular momentum number $m_{j}$ is fixed and there are two AC Stark shifts, for $\left|m_{j}\right|=1 / 2$ and 3/2. The Stark shifts of the $(n+1) d_{j}(n-2) f_{7 / 2}$ state are easily calculated using a Floquet approach 


$$
\begin{array}{cc}
E=0 & E \neq 0 \\
(n+1) d_{j}(n-2) f_{7 / 2}
\end{array}
$$

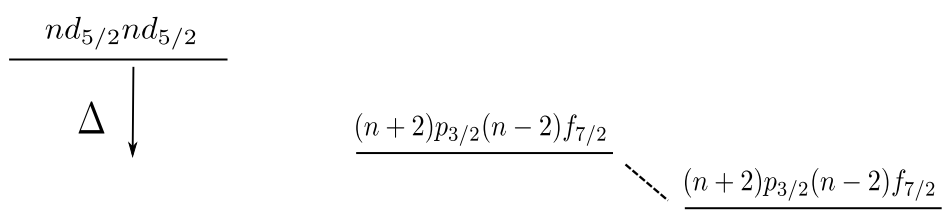

Figure 3.2: AC Stark shift of the $(n+1) d_{j}(n-2) f$ and $(n+2) p_{3 / 2}(n-2) f_{7 / 2}$ states and are given by [19]

$$
\Delta W_{(n+1) d_{j}(n-2) f_{7 / 2}}=\frac{\left(\left\langle(n+1) d_{5 / 2} m_{j}\left|\mu_{z}\right|(n+2) p_{3 / 2} m_{j}\right\rangle E\right)^{2}}{4 \Delta}
$$

For $\left|m_{j}\right|=1 / 2$ and $3 / 2$ the Stark shifts are given by

$$
\Delta W_{(n+1) d_{j}(n-2) f_{7 / 2}}=\frac{\gamma n^{4} E^{2}}{\Delta}
$$

with $\gamma=0.0801$ and 0.0534 for $\left|m_{j}\right|=1 / 2$ and $3 / 2$, respectively, when $n=39$. The constant $\gamma$ includes all the angular factors and the scaling of the radial matrix elements, and $\gamma$ ranges from 0.07997 to 0.0802 for $\left|m_{j}\right|=1 / 2$ and 0.05331 to 0.05347 for $\left|m_{j}\right|=3 / 2$ as $n$ is increased from 35 to 42 . The radial matrix elements for $\left\langle(n+1) d_{5 / 2}\left|\mu_{z}\right|(n+2) p_{3 / 2}\right\rangle$ are given in Ref. [23]. 
Lastly, it is worthwhile to point out that while minimal shift with Rydberg atom density is observed experimentally, the Van der Waals shifts of $\left|n d_{5 / 2} n d_{5 / 2}\right\rangle$ and $\left|(n+2) p_{3 / 2}(n-2) f\right\rangle$ states are not zero and may need to be taken into account at higher densities. It is straightforward to calculate the Van der Waals shifts by calculating the eigenvalues of the two-by-two Hamiltonian matrix with $\left|n d_{5 / 2} n d_{5 / 2}\right\rangle$ and $\left|(n+2) p_{3 / 2}(n-2) f\right\rangle$ as basis states:

$$
\mathcal{H}=\left(\begin{array}{cc}
0 & V_{d d} \\
V_{d d} & -\Delta
\end{array}\right) .
$$

The eigenvalues are

$$
\lambda_{ \pm}=\frac{-\Delta \pm \sqrt{\Delta^{2}+4 V_{d d}^{2}}}{2}
$$

The shifts are given by the differences between $\lambda_{ \pm}$and the eigenvalues without dipole-dipole interaction (i.e. 0 and $-\Delta$ ). For our typical maximum density of $5 \times 10^{8} \mathrm{~cm}^{-3}, V_{d d} \sim 5 \mathrm{MHz}$, which leads to the Van der Waals shift of $0.08 \mathrm{MHz}$ when $n=44$, where $\Delta$ has the smallest value of $62.88 \mathrm{MHz}$ among the states we used for the experiment. Since the shift is much smaller than the FWHM of the resonant peaks, we ignore the Van der Waals shifts.

When $n=43, \Delta=8 \mathrm{MHz}$, the same density leads to the shift of $0.58 \mathrm{MHz}$, which is much greater than the shifts for other states. However, we skip $n=43$, because there is a large adiabatic population transfer to higher $n$ during the field pulse which completely overshadows the resonant signal. 


\subsection{Experimental Procedure}

In the experiment ${ }^{85} \mathrm{Rb}$ atoms are trapped in a magneto-optical trap (MOT) which is vapor loaded. The MOT provides a steady population of $\mathrm{Rb}$ atoms in the $5 p_{3 / 2}$ state. Atoms are excited to the $n d_{5 / 2}$ state by a $10 \mu J 480 \mathrm{~nm}$ laser pulse which is generated by pulse amplifying, at a $20 \mathrm{~Hz}$ repetition rate, the output of tapered amplifier seeded by a $960 \mathrm{~nm}$ diode laser and then frequency doubling it. The optical pulse is $10 \mathrm{~ns}$ long and has a bandwidth of $150 \mathrm{MHz}$. Approximately $4 \mathrm{~ms}$ before the pulsed laser excitation, the trap magnetic fields are switched off to reduce the residual field in the MOT to less than $50 \mathrm{mG}$ during the experiment. Subsequent to laser excitation, the atoms are exposed to a $1 \mu s$ long microwave pulse to drive the $n d_{5 / 2} n d_{5 / 2} \rightarrow(n+1) d_{j}(n-2) f_{7 / 2}$ or $n d_{5 / 2} n d_{5 / 2} \rightarrow(n+2) p_{3 / 2}(n-1) d_{5 / 2}$ transition.

Fifty nanoseconds after the end of the microwave pulse, a 700 ns rise time voltage pulse is applied to the rods to field ionize the Rydberg atoms and drive the resulting ions to a microchannel plate (MCP) detector. The signal from the MCP is recorded with either a gated integrator or an oscilloscope and stored in a computer for later analysis.

The cloud of cold $\mathrm{Rb}$ atoms is held at the center of four vertical rods which pass through the corners of a horizontal square $18 \mathrm{~mm}$ on a side. The rods allow the application of the field ionization pulse. The density of the Rydberg atoms in the MOT is determined by the methods described in Section 2.1.6. In these experiments, the maximum value of the density, $\rho_{0}$, is $4 \times 10^{8} \mathrm{~cm}^{-3}$, and the density measurement uncertainty is a factor of three.

The microwaves are generated in an Agilent 83622B synthesizer, which has a 
maximum frequency of $20 \mathrm{GHz}$, and a General Microwave DM862B switch is used to form the microwaves into $1 \mu s$ long pulses. A Hewlett Packard (HP) 83554A passive doubler is used for the 26.5 to $40 \mathrm{GHz}$ range, covering $37 \leq n \leq 44$, and a Narda DBS4060X410 active quadrupler is used for the 40 to $60 \mathrm{GHz}$ range, covering $35 \leq$ $n \leq 37$. The relative microwave power is controlled in the final waveguide with a HP R832A or U832A precision attenuator. The microwaves have horizontal polarization and propagate from a horn outside the vacuum system through a window to the MOT volume. The vertical rods used to apply field ionization pulse scatter the microwaves to some extent, and this may result in the polarization's not being perfectly linear. As mentioned earlier, there is an AC Stark shift due to near resonance of the microwaves to the $(n+2) p_{3 / 2} \rightarrow(n+1) d_{j}$ transition. It is straightforward to extrapolate the location of the resonance peaks to zero microwave power, and the power shift is used to calibrate absolutely the microwave fields over the range of frequencies employed.

To field ionize atoms in states above $n=41$ we apply a positive voltage pulse to the rods farther from the MCP while the two rods nearer the MCP are grounded. To ionize atoms in states of $37 \leq n \leq 41$ we also apply a negative voltage pulse with the same 700 ns risetime to the rods nearer to the MCP. This two pulse scheme is implemented because the output of one circuit for generating the pulse with a rapid initial rise is limited to $500 \mathrm{~V}$, which is not enough to ionize the Rydberg states of $n \leq 41$. The field ionization pulse has a rapid initial rise and is similar to the fast pulse used for the experiment by Han [10]. Using this type of pulse with rapid initial rise allows us to suppress adiabatic transitions through molecular avoided crossings on the rising edge of the field pulse. Such transitions, which occur with slowly rising 
field pulses [10,21] (and also from private communication with Noel and Brune), lead to field ionization signals almost identical to the resonant signals we wish to detect. Since they originate from pairs of closely spaced atoms, they artificially suppress the resonant signals due to the processes of Eqs. (3.1) and (3.3). For $35 \leq n \leq 37$ we have used a field ionization pulse which has a higher amplitude but a slow initial rise [10].

\subsection{Results and Analysis}

Our signals are time resolved state-selective field ionization signals. As an example, ionization signals from atoms excited to $39 d_{5 / 2}$ with no microwaves (broken line) and with microwaves tuned to the $39 d_{5 / 2} 39 d_{5 / 2} \rightarrow 40 d_{5 / 2} 37 f_{7 / 2}$ resonance (solid line) are shown in Fig. 3.3. Time $t=0$ is when the field ionization circuit is triggered. The large peak at $t=4.36 \mu \mathrm{s}$ is from atoms in the $39 d_{5 / 2}$ state. With microwaves on, a peak earlier in time at $t=4.29 \mu \mathrm{s}$, corresponding to atoms in the $40 d_{5 / 2}$ state appears, and a decrease in the $39 d_{5 / 2}$ signal is observed. Careful examination of Fig. 3.3 reveals a small signal at $t=4.29 \mu$ s with no microwaves due to the non adiabatic transitions during the field pulse. 


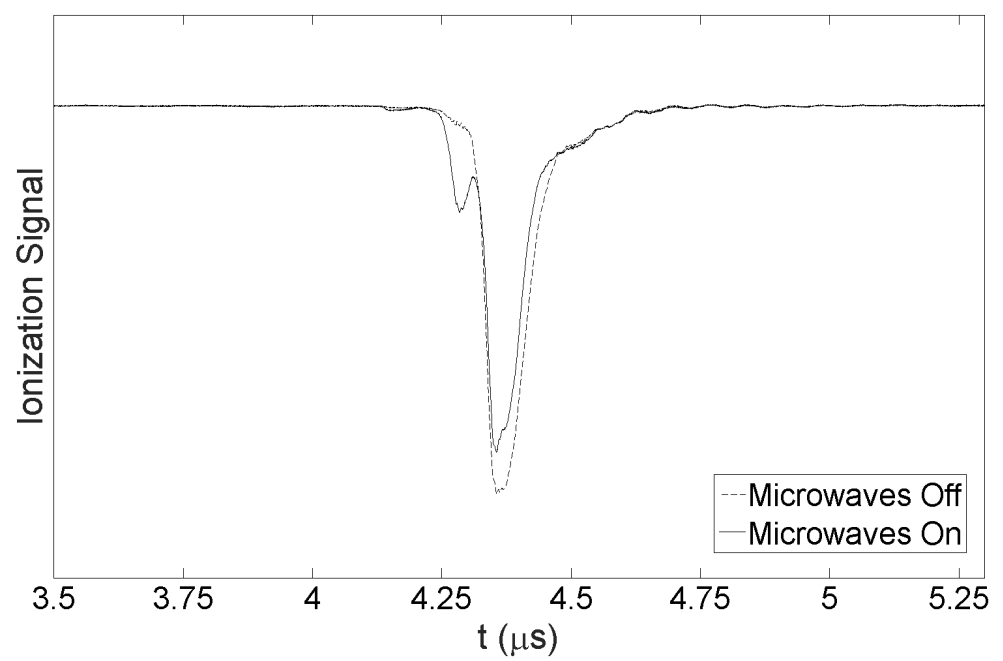

Figure 3.3: Field ionization signal for the $39 d_{5 / 2} 39 d_{5 / 2} \rightarrow 40 d_{5 / 2} 37 f_{7 / 2}$ transition with microwaves off (broken line) and microwaves on (solid line). The microwaves are tuned to $33.613 \mathrm{GHz}$, the resonance frequency for $39 d_{5 / 2} 39 d_{5 / 2} \rightarrow 40 d_{5 / 2} 37 f_{7 / 2}$ transition.

By setting the gate of the integrator on the $40 d_{j}$ signal at $t=4.29 \mu$ s and sweeping the microwave frequency over many shots of the laser we obtain the $39 d_{5 / 2} 39 d_{5 / 2} \rightarrow$ $40 d_{j} 37 f_{7 / 2}$ traces of Fig. 3.4, which show the resonances for a range of microwave field amplitudes. There is no signal at the frequencies of the $41 p_{3 / 2} \rightarrow 40 d_{3 / 2}$ and $41 p_{3 / 2} \rightarrow 40 d_{5 / 2}$ atomic transitions (Those atomic transitions are not within the scan range for Fig. 3.4). As the microwave field amplitude is raised, the transitions exhibit AC Stark shifts to higher frequency as well as splitting and broadening. The origin of the AC Stark shift is the near resonance with the atomic $41 p_{3 / 2}-40 d_{j}$ transition. We attribute the splitting to the difference in the AC Stark shifts of the $40 d_{5 / 2}$ state of $\left|m_{j}\right|=1 / 2$ and $\left|m_{j}\right|=3 / 2$, as discussed earlier. The broadening is due, at least in part, to inhomogeneities in the microwave field. The relative microwave fields of 
the traces shown in Fig. 3.4 are easily determined from the setting of the attenuator, and the absolute fields are determined by comparing the observed shifts to those calculated assuming the shifts to originate from the $41 p_{3 / 2}-40 d_{j}$ transitions, as described in an earlier section.

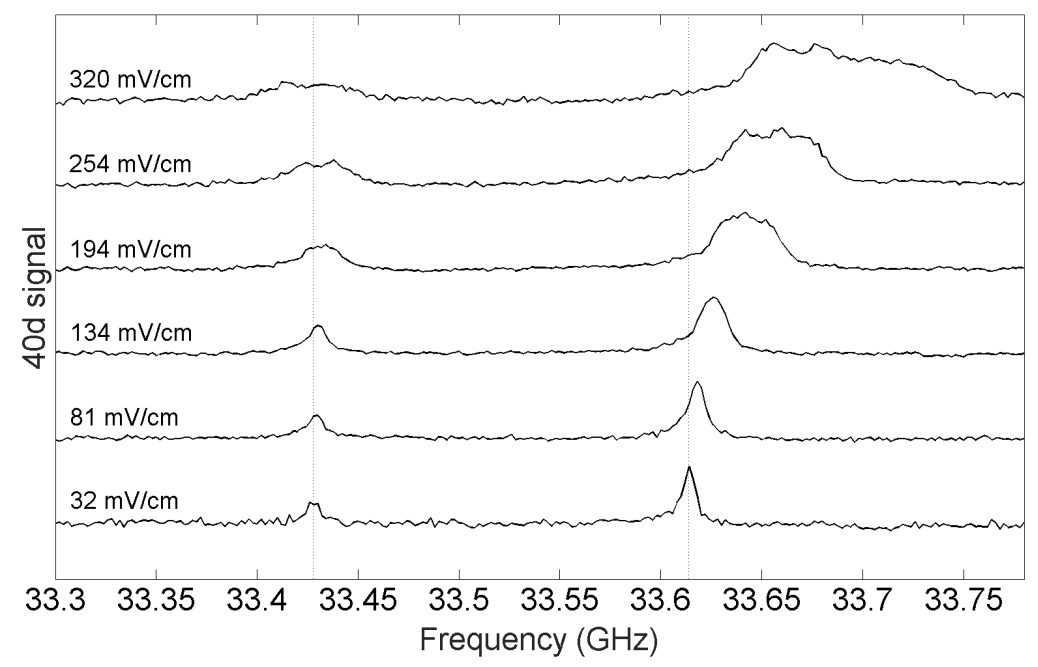

Figure 3.4: Observed $39 d_{5 / 2} 39 d_{5 / 2} \rightarrow 40 d_{j} 37 f_{7 / 2}$ resonances for a range of microwave field amplitudes. The dotted lines represent the calculated resonance frequencies for the transitions at zero microwave power and $R=\infty$.

Fig. 3.5 shows the $45 \mathrm{~d}$ signal vs. microwave frequency for the $44 d_{5 / 2} 44 d_{5 / 2} \rightarrow$ $45 d_{j} 42 f$ transitions for a range of microwave field amplitudes. Again, there is no signal at the frequencies of the $46 p_{3 / 2} \rightarrow 45 d_{3 / 2}$ and $46 p_{3 / 2} \rightarrow 45 d_{5 / 2}$ atomic transitions, indicated by the arrows. For $n=44, \Delta<0$ and an AC Stark shift of the resonances in the opposite direction is observed. As in Fig. 3.4, the relative microwave fields are determined from the attenuator, and they are put on an absolute basis using the $\mathrm{AC}$ Stark shift. 


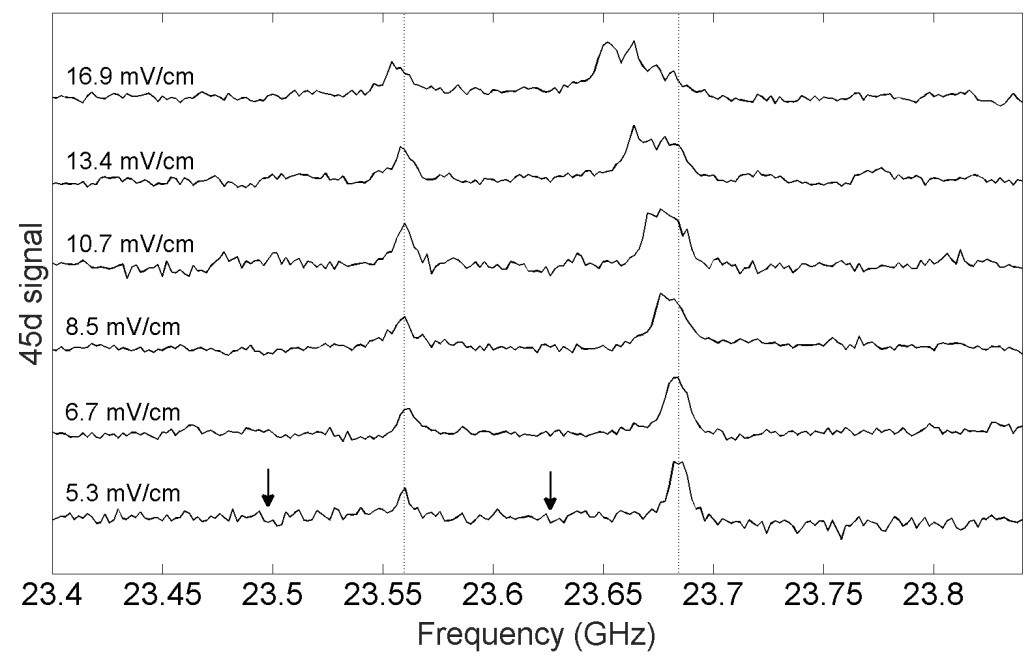

Figure 3.5: Observed $44 d_{5 / 2} 44 d_{5 / 2} \rightarrow 45 d_{j} 42 f_{7 / 2}$ resonances for a range of microwave field amplitudes. The dotted lines represent the calculated resonance frequencies for the transitions at zero microwave power and $R=\infty$.

To obtain the zero microwave power resonance frequency for a transition, the frequency of the resonance peak at different microwave field amplitudes is extrapolated back to zero power. Fig. 3.6 shows an example of the extrapolation, for the $37 d_{5 / 2} 37 d_{5 / 2} \rightarrow 38 d_{5 / 2} 35 f_{7 / 2}$ transition. Tables 3.2 and 3.3 give the zero microwave power frequencies for our measurements. All frequencies agree well with the calculated $R \rightarrow \infty$ values, which are calculated using the quantum defects of Refs. $[11,14]$. 


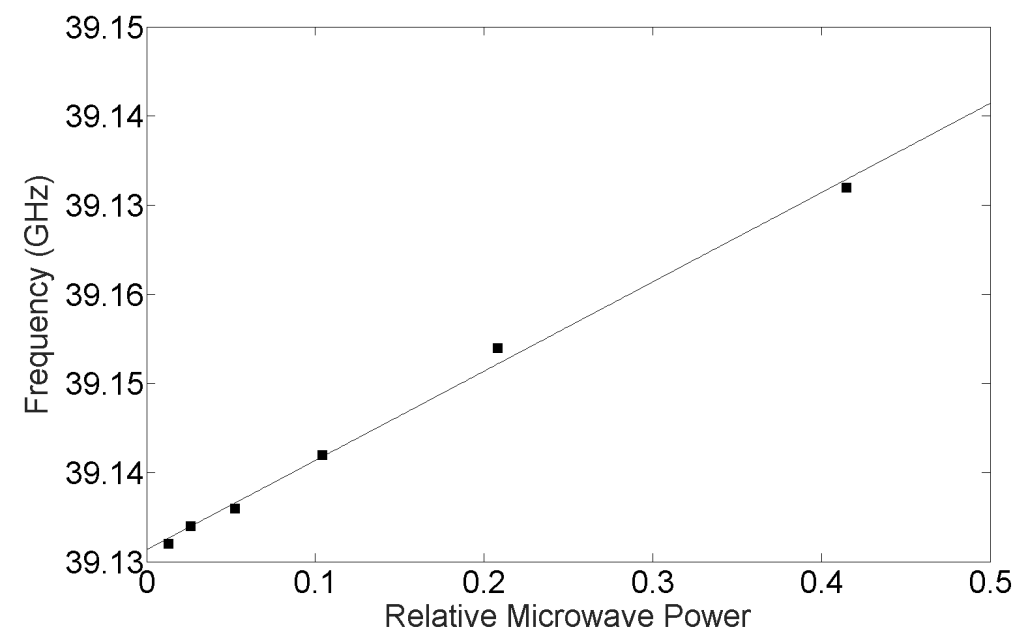

Figure 3.6: Resonance frequency vs. microwave power for the $37 d_{5 / 2} 37 d_{5 / 2} \rightarrow$ $38 d_{5 / 2} 35 f_{7 / 2}$ transition.

\begin{tabular}{cccc}
$n$ & Calculated(MHz) & Observed(MHz) & Difference $(\mathrm{MHz})$ \\
\hline 34 & 49897.9 & 49898.4 & 0.5 \\
35 & 45916.0 & 45915.0 & 1.0 \\
36 & 42344.0 & 42341.0 & 3.0 \\
37 & 39131.0 & 39130.7 & 0.3 \\
38 & 36233.3 & 36232.8 & 0.5 \\
39 & 33613.3 & 33613.6 & -0.3 \\
40 & 31238.7 & 31238.3 & 0.4 \\
41 & 29081.9 & 29080.0 & 1.9 \\
42 & 27117.9 & 27116.0 & 1.9 \\
44 & 23688.6 & 23689.6 & -1.0
\end{tabular}

Table 3.2: Resonance frequencies for the $n d_{5 / 2} n d_{5 / 2} \rightarrow(n+1) d_{5 / 2}(n-2) f_{7 / 2}$ transitions 


\begin{tabular}{cccc}
$n$ & Calculated $(\mathrm{MHz})$ & Observed $(\mathrm{MHz})$ & Difference $(\mathrm{MHz})$ \\
\hline 34 & 49618.3 & 49617.4 & 0.9 \\
35 & 45659.8 & 45659.6 & 0.2 \\
36 & 42108.8 & 42106.2 & 2.6 \\
37 & 38914.4 & 38911.9 & 2.5 \\
38 & 36033.5 & 36030.8 & 2.7 \\
39 & 33428.6 & 33427.7 & 0.9 \\
40 & 31067.6 & 31066.2 & 1.4 \\
41 & 28922.9 & 28920.6 & 2.3 \\
42 & 26970.2 & 26968.2 & 2.0 \\
44 & 23560.3 & 23563.0 & -2.7
\end{tabular}

Table 3.3: Resonance frequencies for the $n d_{5 / 2} n d_{5 / 2} \rightarrow(n+1) d_{3 / 2}(n-2) f_{7 / 2}$ transitions

While there is an evident microwave power shift, there is minimal shift with Rydberg atom density. As an example, Fig. 3.7 shows scans of the $40 d_{j}$ signal vs. microwave frequency for the $39 d_{5 / 2} 39 d_{5 / 2} \rightarrow 40 d_{j} 37 f_{7 / 2}$ transitions for a range of Rydberg atom densities. 


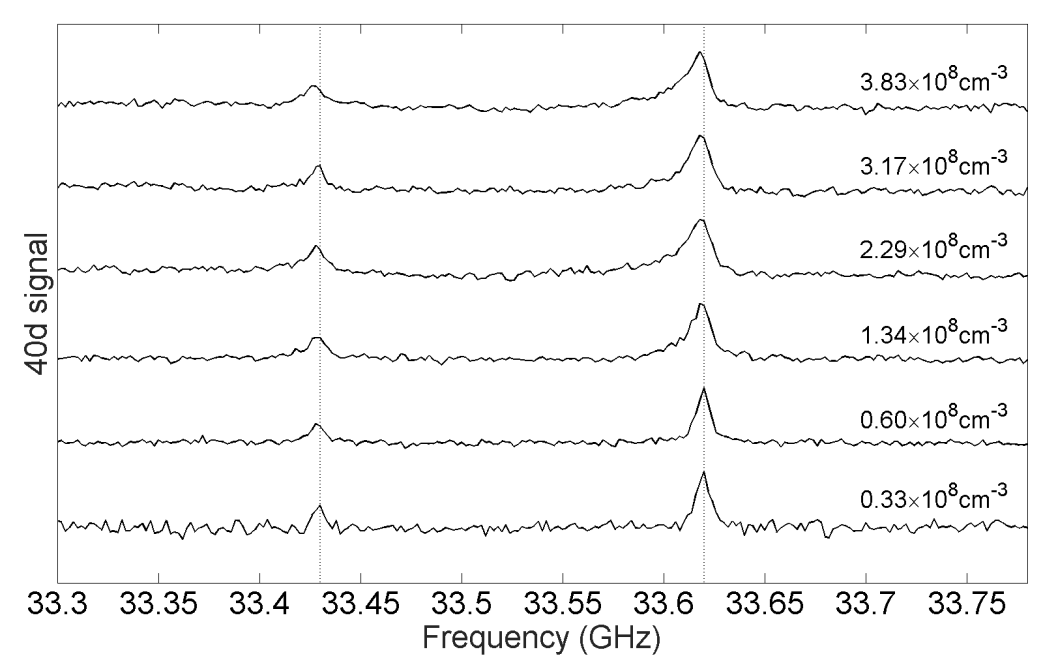

Figure 3.7: Observed $39 d_{5 / 2} 39 d_{5 / 2} \rightarrow 40 d_{j} 37 f_{7 / 2}$ resonances for a range of peak Rydberg atom densities. The dotted lines represent the calculated resonance frequencies for the transition at zero microwave power and $R=\infty$.

To test the CI model we have measured the FPT from the $n d_{5 / 2} n d_{5 / 2}$ to the $(n+1) d_{j}(n-2) f_{7 / 2}$ states. The model is valid for small FPT. At very high densities other processes begin to occur. For example, transfer to not only the $(n+1) d_{j}$ state but also the $(n+2) d_{j}$ state is observed. Accordingly, we have kept the density below $\rho_{0}=5 \times 10^{8} \mathrm{~cm}^{-3}$, where the observations are consistent with our CI model. To measure the FPT for $37 \leq n \leq 42$, the ratio of the increase in the $(n+1) d_{j}$ signal when microwaves are tuned to resonance to the area of the $n d_{5 / 2}$ signal without the microwaves is used. For $n=44$ even with the fast field ionization pulse there is appreciable population transfer to higher lying states in the field pulse, so we do not include $n=44$ in the FPT measurements. For $35 \leq n \leq 37$ we have used the higher amplitude slow field ionization pulse and measured the depletion of the $n d_{5 / 2}$ signal with the microwaves at the resonance frequency. For $n=37$, the state for which the 
FPT was measured both ways, the fractional depletion of the $37 d_{5 / 2}$ signal is slightly over twice as large as the increase in the $38 d_{j}$ signal (A factor of two is expected, since population must go down in energy to the $35 f_{7 / 2}$ state). Using the same factor used to normalize the $n=37$ depletion data we normalize the $n=35$ and 36 depletion data for inclusion in Fig. 3.8 and Fig. 3.9. For each state, the absolute microwave field amplitude is obtained by using the AC Stark shift, as described previously.

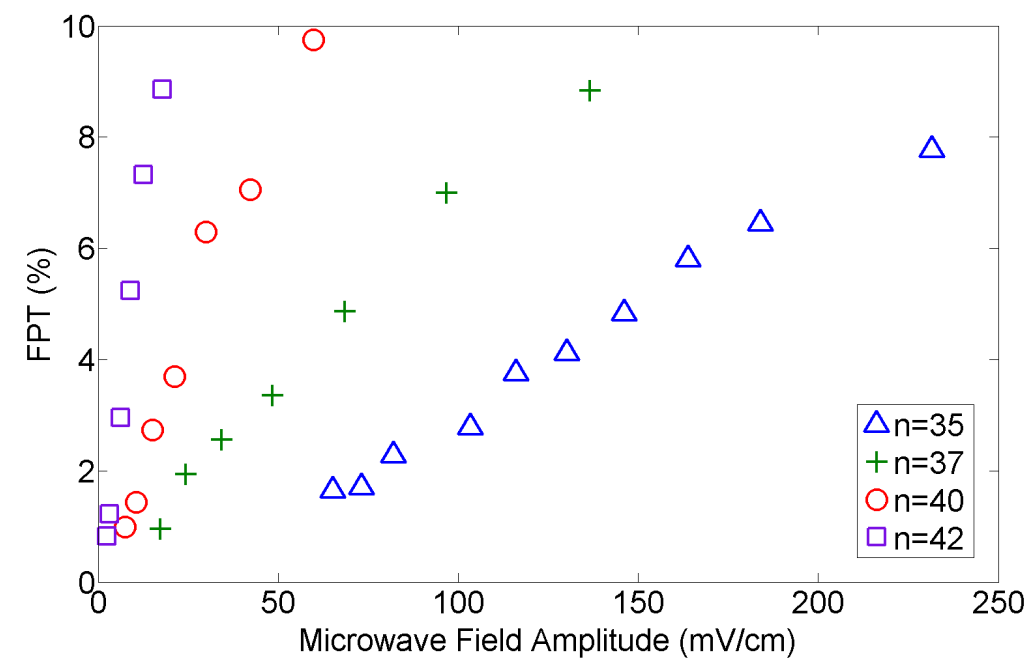

Figure 3.8: FPT vs. microwave field amplitude for $n=35(\triangle), n=37(+), n=40$ (०), and $n=42(\square)$ at similar densities of $\rho_{0}=1.7 \times 10^{8} \mathrm{~cm}^{-3}$ 


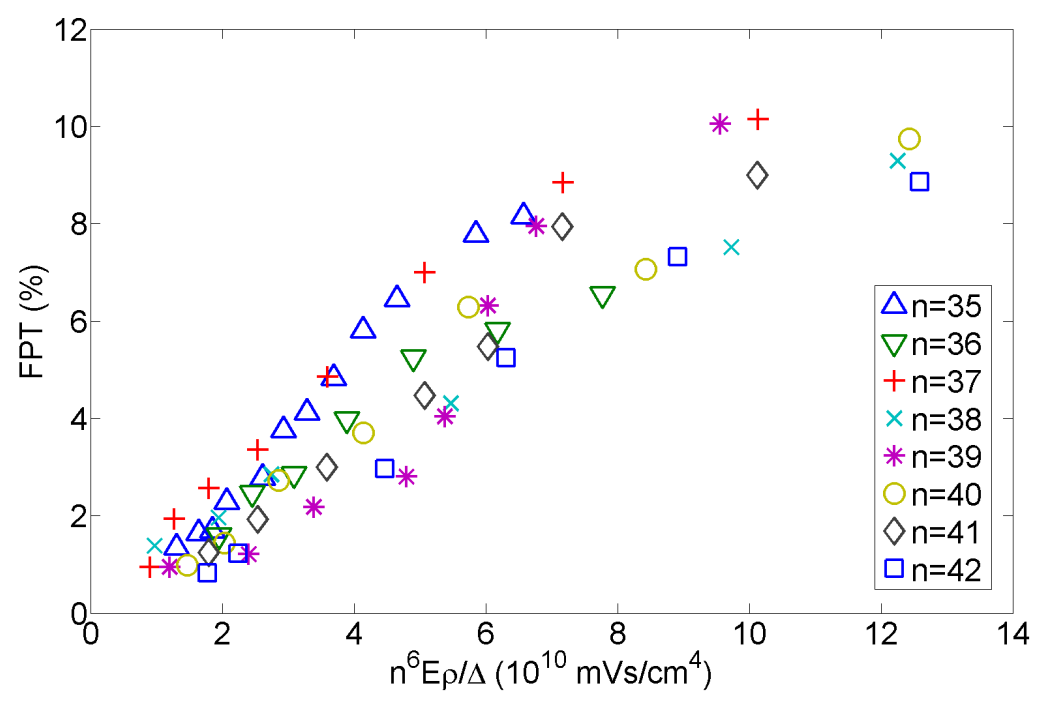

Figure 3.9: FPT vs. $\frac{n^{6} \rho E}{\Delta}$ for $35 \leq n \leq 42$

Fig. 3.8 shows the FPT vs. microwave field amplitude for $n=35,37,40$, and 42 at similar densities of $\rho_{0}=1.7 \times 10^{8} \mathrm{~cm}^{-3}$. Between $n=35$ and 42 , the microwave field amplitude required to produce the same FPT drops by more than a factor of thirty. As suggested by Eq. (3.13), plotting the FPT vs $\frac{n^{6} \rho E}{|\Delta|}$ should result in the data points' falling on the same straight line. As shown by Fig. 3.9, the points are close to falling on the same line, but at higher $n$ the points are systematically low. This deviation from the expectation based on Eq. (3.13) may be due to a slightly larger adiabatic population transfer to higher $n$ during the field pulse, which is more likely at higher $n$ and suppresses the resonant signal. It is primarily the decrease in $\Delta$ which leads to the larger adiabatic transfer as $n$ is raised. In any event, Fig. 3.9 demonstrates that the CI description of the process is quite good.

Since the CI couples the $n d_{5 / 2} n d_{5 / 2}$ state to the $(n+2) p_{3 / 2}(n-2) f_{7 / 2}$ state it should be possible to drive transitions in which the $(n-2) f_{7 / 2}$ atom undergoes the 
transition while the $(n+2) p_{3 / 2}$ atom is a spectator, as shown by the energy level diagram of Fig. 3.10. To verify that such transitions are possible we have driven the $39 d_{5 / 2} 39 d_{5 / 2} \rightarrow 41 p_{3 / 2} 38 d_{5 / 2}$ transition of Fig. 3.10. As shown in Fig. 3.10, the transition is to a molecular state lower in energy, but the atomic $41 p_{3 / 2}$ state lies above the initial $39 d_{5 / 2}$ state, so at resonance the $41 p_{3 / 2}$ signal appears earlier in time than the $39 d_{5 / 2}$ signal, approximately as shown in Fig. 3.3. The observed resonance is shown in Fig. 3.11. The location of the peak, at $43.922 \mathrm{GHz}$ (extrapolated to zero microwave power), is in good agreement with the calculated $R=\infty$ interval of $43.9213 \mathrm{GHz}$. However, the origin of the pedestal seen in Fig. 3.11 is not yet understood.

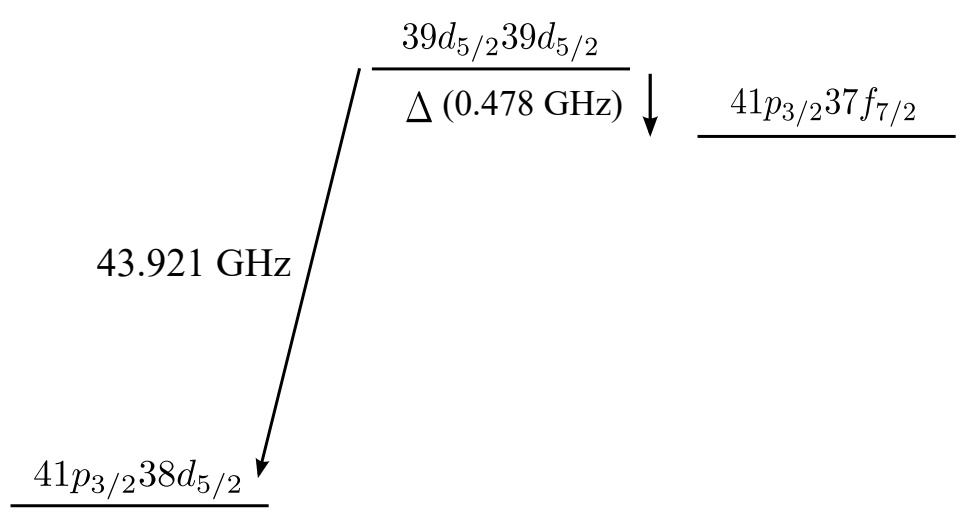

Figure 3.10: Energy level diagram for the $39 d_{5 / 2} 39 d_{5 / 2} \rightarrow 41 p_{3 / 2} 38 d_{5 / 2}$ transition 


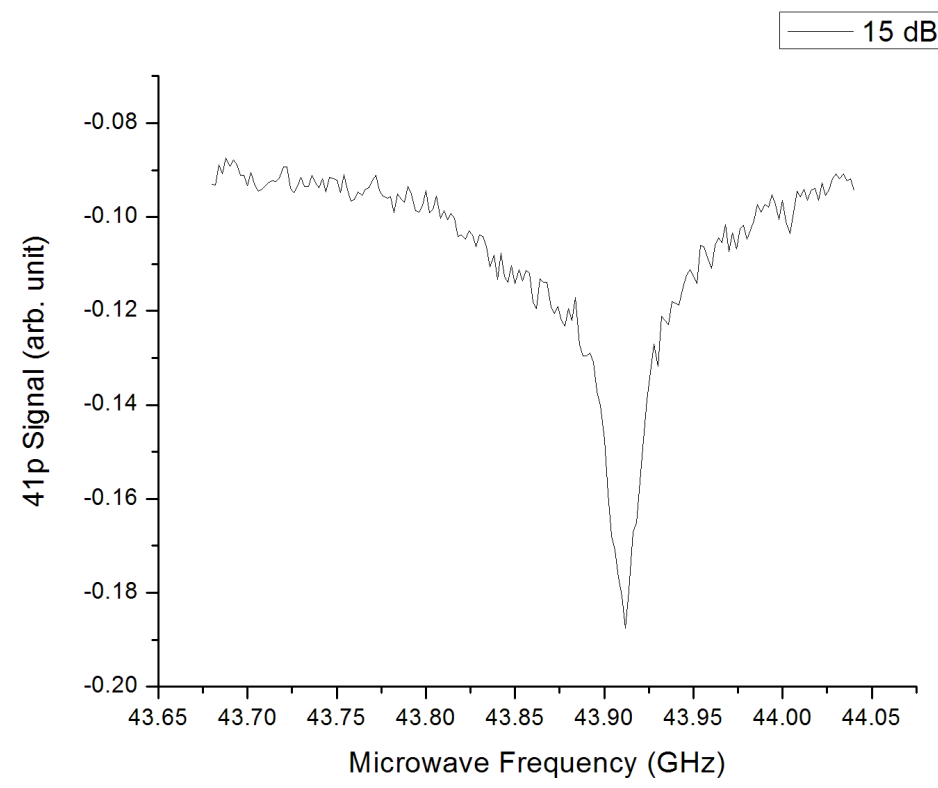

Figure 3.11: Observed $39 d_{5 / 2} 39 d_{5 / 2}$ to $41 p_{3 / 2} 38 d_{5 / 2}$ transition

\subsection{Conclusion}

We have observed resonant microwave transitions between pairs of atoms in which a single microwave photon is absorbed, yet both of the constituent atoms in the molecule change state. Specifically, we have observed the processes $n d_{5 / 2} n d_{5 / 2} \rightarrow$ $(n+1) d_{j}(n-2) f_{7 / 2}$ and $n d_{5 / 2} n d_{5 / 2} \rightarrow(n+2) p_{3 / 2}(n-1) d_{5 / 2}$. These transitions are allowed due to the dipole-dipole induced configuration interaction between the $n d_{5 / 2} n d_{5 / 2}$ state and the energetically nearby $(n+2) p_{3 / 2}(n-2) f_{7 / 2}$ state, which admixes some of the latter into the former. The strengths of the transitions are in good agreement with a simple CI description of the process. 


\section{REFERENCES}

[1] Anderson, W. R., Veale, J. R., \& Gallagher, T. F. (1998). Resonant dipole-dipole energy transfer in a nearly frozen rydberg gas. Phys. Rev. Lett., 80, 249-252.

[2] Bendkowsky, V., Butscher, B., Nipper, J., Shaffer, J. P., Löw, R., \& Pfau, T. (2009). Observation of ultralong-range rydberg molecules. Nature, 458, 1005-1008.

[3] Boisseau, C., Simbotin, I., \& Côté, R. (2002). Macrodimers: Ultralong range rydberg molecules. Phys. Rev. Lett., 88, 133004.

[4] Deiglmayr, J., Saßmannshausen, H., Pillet, P., \& Merkt, F. (2014). Observation of dipole-quadrupole interaction in an ultracold gas of rydberg atoms. Phys. Rev. Lett., 113, 193001.

[5] Edmonds, A. (1960). Angular Momentum in Quantum Mechanics. Princeton, NJ: Princeton University Press.

[6] Faoro, R., Pelle, B., Zuliani, A., Cheinet, P., Arimondo, E., \& Pillet, P. (2015). Borromean three-body fret in frozen rydberg gases. Nat Commun, 6 .

[7] Farooqi, S. M., Tong, D., Krishnan, S., Stanojevic, J., Zhang, Y. P., Ensher, J. R., Estrin, A. S., Boisseau, C., Côté, R., Eyler, E. E., \& Gould, P. L. (2003). Long-range molecular resonances in a cold rydberg gas. Phys. Rev. Lett., 91, 183002.

[8] Greene, C. H., Dickinson, A. S., \& Sadeghpour, H. R. (2000). Creation of polar and nonpolar ultra-long-range rydberg molecules. Phys. Rev. Lett., 85, 2458-2461.

[9] Gurian, J. H., Cheinet, P., Huillery, P., Fioretti, A., Zhao, J., Gould, P. L., Comparat, D., \& Pillet, P. (2012). Observation of a resonant four-body interaction in cold cesium rydberg atoms. Phys. Rev. Lett., 108, 023005.

[10] Han, J. \& Gallagher, T. F. (2008). Field ionization of the frozen rydberg gas. Phys. Rev. A, 77, 015404.

[11] Han, J., Jamil, Y., Norum, D. V. L., Tanner, P. J., \& Gallagher, T. F. (2006). $\mathrm{Rb} n f$ quantum defects from millimeter-wave spectroscopy of cold ${ }^{85} \mathrm{Rb}$ rydberg atoms. Phys. Rev. A, 74, 054502.

[12] Kiffner, M., Li, W., \& Jaksch, D. (2013). Three-body bound states in dipoledipole interacting rydberg atoms. Phys. Rev. Lett., 111, 233003. 
[13] Kiffner, M., Park, H., Li, W., \& Gallagher, T. F. (2012). Dipole-dipole-coupled double-rydberg molecules. Phys. Rev. A, 86, 031401.

[14] Li, W., Mourachko, I., Noel, M. W., \& Gallagher, T. F. (2003). Millimeter-wave spectroscopy of cold rb rydberg atoms in a magneto-optical trap: Quantum defects of the $n s, n p$, and $n d$ series. Phys. Rev. A, 67, 052502.

[15] Mourachko, I., Comparat, D., de Tomasi, F., Fioretti, A., Nosbaum, P., Akulin, V. M., \& Pillet, P. (1998). Many-body effects in a frozen rydberg gas. Phys. Rev. Lett., 80, 253-256.

[16] Overstreet, K. R., Schwettmann, A., Tallant, J., Booth, D., \& Shaffer, J. P. (2009). Observation of electric-field-induced cs rydberg atom macrodimers. Nat Phys, 5, 581-585.

[17] Pillet, P., Kachru, R., Tran, N. H., Smith, W. W., \& Gallagher, T. F. (1987). Radiative rydberg-atom rydberg-atom collisions in the strong-field regime. Phys. Rev. A, 36, 1132-1147.

[18] Samboy, N., Stanojevic, J., \& Côté, R. (2011). Formation and properties of rydberg macrodimers. Phys. Rev. A, 83, 050501.

[19] Shirley, J. H. (1965). Solution of the schrödinger equation with a hamiltonian periodic in time. Phys. Rev., 138, B979-B987.

[20] Tallant, J., Rittenhouse, S. T., Booth, D., Sadeghpour, H. R., \& Shaffer, J. P. (2012). Observation of blueshifted ultralong-range $\mathrm{cs}_{2}$ rydberg molecules. Phys. Rev. Lett., 109, 173202.

[21] Teixeira, R. C., Hermann-Avigliano, C., Nguyen, T. L., Cantat-Moltrecht, T., Raimond, J. M., Haroche, S., Gleyzes, S., \& Brune, M. (2015). Microwaves probe dipole blockade and van der waals forces in a cold rydberg gas. Phys. Rev. Lett., $115,013001$.

[22] Tong, D., Farooqi, S. M., Stanojevic, J., Krishnan, S., Zhang, Y. P., Côté, R., Eyler, E. E., \& Gould, P. L. (2004). Local blockade of rydberg excitation in an ultracold gas. Phys. Rev. Lett., 93, 063001.

[23] Walker, T. G. \& Saffman, M. (2008). Consequences of zeeman degeneracy for the van der waals blockade between rydberg atoms. Phys. Rev. A, 77, 032723.

[24] Yu, Y., Park, H., \& Gallagher, T. F. (2013). Microwave transitions in pairs of rb rydberg atoms. Phys. Rev. Lett., 111, 173001. 


\section{Chapter 4}

\section{Multiphoton Molecular Transitions}

\subsection{Introduction}

Microwave fields provide a convenient way to probe and control Rydberg atoms and their interactions, as shown by a few examples. The observation of the motion of $\mathrm{Rb}$ Rydberg atoms on a repulsive van der Waals potential has been observed by Teixeira et al.[6] who followed the changing frequency shift of a microwave transition. Microwaves and radio frequency fields have been used to control Forster resonant energy transfer, recently in cold Rydberg atom samples by van Ditzhuizen[7], and some time ago in samples of room temperature atoms by Pillet et al.[4]. Microwaves have also been used to select pairs of atoms with well defined dipole-dipole interactions. In Chapter 3, the microwave spectroscopy of pairs of Rydberg atoms was reported. In particular, we observed transitions in which a pair of $\mathrm{Rb} n d_{5 / 2}$ atoms, in the $n d_{5 / 2} n d_{5 / 2}$ molecular state, absorbed a single microwave photon and underwent 
the transition to the $(n+1) d_{j}(n-2) f$ state. In spite of the fact that only one photon was absorbed, both atoms changed state. We described the process in terms of a configuration interaction (CI) model, in which the dipole-dipole interaction induced admixture of the energetically nearby $(n+2) p_{3 / 2}(n-2) f$ state into the $n d_{5 / 2} n d_{5 / 2}$ state allows the microwave transition to the $(n+1) d_{j}(n-2) f$ state.

In this chapter, we describe the extension of the observations to other single photon transitions between pairs of Rb Rydberg atoms, and to multiphoton transitions between pairs of atoms. All of these processes can be described in terms of Forster resonant dipole-dipole energy transfers between Floquet, or microwave dressed, states. In this approach we treat the interaction of the atoms with the microwave field first, and then the dipole-dipole interaction between the resulting Floquet states. This approach is similar to that followed by van Ditzhuizen et al.[7] and Pillet et al.[4], but it differs in that the microwave field interacts with only one of the two atoms of the pair; the other is simply a spectator. The spectator atom is analogous to the spectator Rydberg electron in isolated core excitation of two electron Rydberg atoms. The Floquet approach[5] provides a convenient way to treat multiphoton processes, and it reduces to the CI model for single photon transitions. In the sections which follow we present the Floquet model, describe the experimental approach, present our experimental observations and compare them to the expectations from the model. 


\subsection{Floquet Model of Forster Energy Transfer}

In Fig. 4.1 we show one, two, and three photon microwave transitions from $n d_{5 / 2} n d_{5 / 2}$ pairs to other states of the form $n^{\prime} s_{1 / 2}(n-2) f$ and $(n+3) p_{j}(n-2) f$. Previously we described one photon transitions using a CI model, in which the dipole-dipole interaction of the $n d_{5 / 2} n d_{5 / 2}$ state with the nearby $(n+2) p_{3 / 2}(n-2) f$ state admixes some of the latter into the nominal $n d_{5 / 2} n d_{5 / 2}$ state. With this admixture a relatively weak microwave field can drive the $n d_{5 / 2} n d_{5 / 2}$ to $(n+3) s_{1 / 2}(n-2) f$ transition. In the case of the two and three photon transitions, a stronger microwave field is required, and except for the most closely spaced pairs, the interaction with the microwave field is much stronger than the dipole-dipole interaction. Accordingly, our approach is to treat the interaction of the atoms with the microwave field first, using a Floquet approach and ignoring the dipole-dipole interaction, and then introduce the dipole-dipole interaction between the resulting Floquet states. When the microwave frequency brings Floquet states into degeneracy, Forster resonant energy transfer occurs due to the dipole-dipole interaction. For the case in which a single microwave photon is absorbed or emitted the Floquet approach reduces to the result given by the CI approach used previously.

For concreteness we consider the system shown in Fig. 4.1. Specifically the one, 


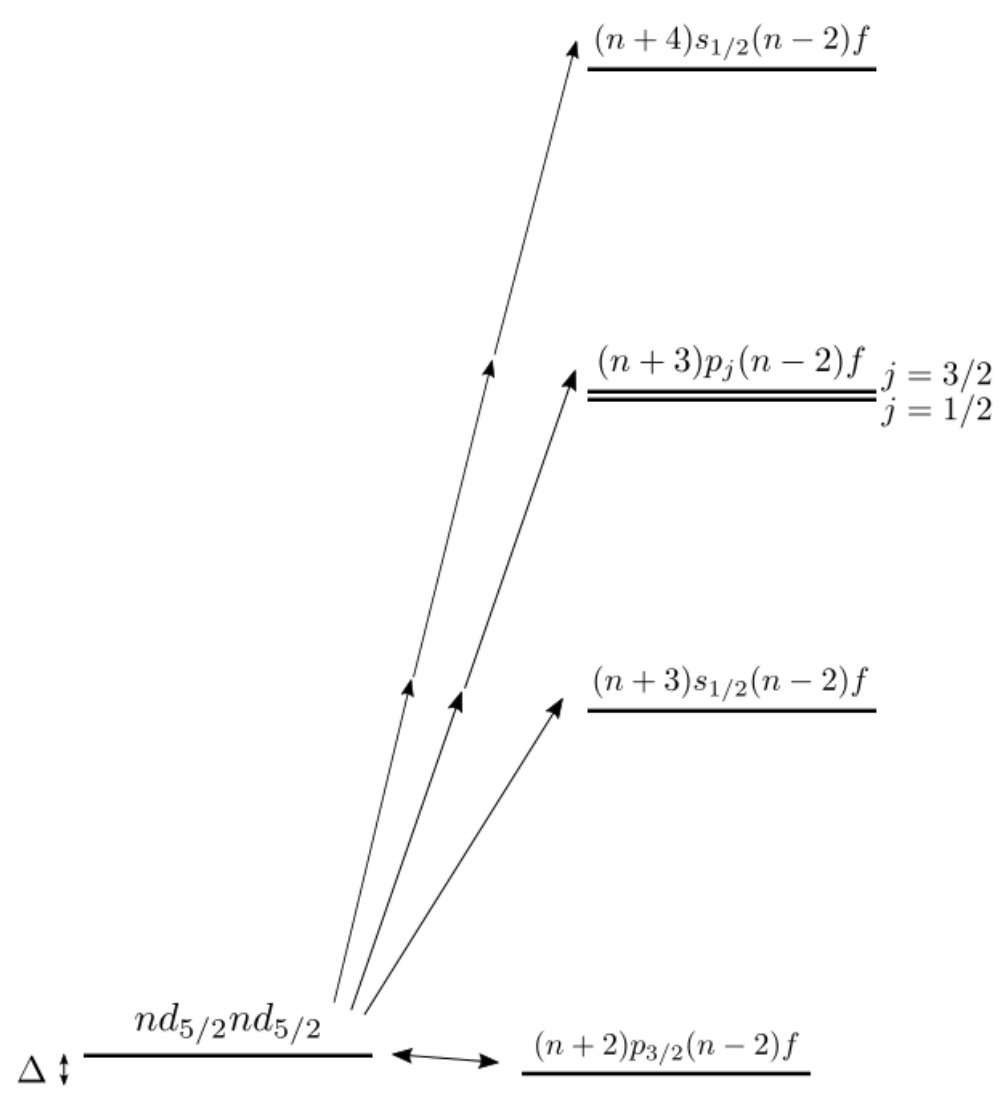

Figure 4.1: One, two, and three photon microwave transitions from $n d_{5 / 2} n d_{5 / 2}$ state. The transitions to $(n+3) p_{j}(n-2) f$ states are two-photon transitions, and the transition to $(n+4) s_{1 / 2}(n-2) f$ state is a three-photon transition. The diagram is approximately to scale. 
two, and three microwave photon transitions

$$
\begin{aligned}
n d_{5 / 2} n d_{5 / 2}+\hbar \omega & \rightarrow(n+3) s_{1 / 2}(n-2) f \\
n d_{5 / 2} n d_{5 / 2}+2 \hbar \omega & \rightarrow(n+3) p_{1 / 2}(n-2) f \\
n d_{5 / 2} n d_{5 / 2}+2 \hbar \omega & \rightarrow(n+3) p_{3 / 2}(n-2) f \\
n d_{5 / 2} n d_{5 / 2}+3 \hbar \omega & \rightarrow(n+4) s_{1 / 2}(n-2) f
\end{aligned}
$$

All of these transitions are allowed due to the dipole-dipole interaction of the $n d_{5 / 2} n d_{5 / 2}$ state with the nearby $(n+2) p_{3 / 2}(n-2) f$ state, as shown by the double headed arrow in Fig. 4.1. We ignore the $(n+2) p_{1 / 2}(n-2) f$ state since it is not coupled to the $n d_{5 / 2} n d_{5 / 2}$ state by the dipole-dipole interaction and its inclusion does not significantly affect the Floquet levels at the resonant frequencies.

Unless stated otherwise we use atomic units, and for compactness in notation we introduce the shorthand

$$
\begin{array}{r}
n d_{5 / 2} \rightarrow d \\
(n+2) p_{3 / 2} \rightarrow p \\
(n+3) s_{1 / 2} \rightarrow s \\
(n+3) p_{1 / 2} \rightarrow p_{1}^{\prime} \\
(n+3) p_{3 / 2} \rightarrow p_{3}^{\prime} \\
(n+4) s_{1 / 2} \rightarrow s^{\prime} \\
(n-2) f \rightarrow f
\end{array}
$$

With this notation the transition of Eq. (4.1a) is written as $d d+\hbar \omega \rightarrow s f$.

The wave functions for the molecular states are direct products of the two atomic 
wave functions, and we ignore exchange. The energies of the molecular states at $\mathrm{R}=\infty$ are obtained by adding the energies of the two atomic states, which are easily calculated using the known Rb quantum defects. For simplicity in notation we specify energies relative to $W_{d d}$, the energy of the $d d$ state.

The molecular dipole matrix elements which are important for the microwave coupling are

$$
\begin{gathered}
\langle p f|\mu| s f\rangle=\langle p|\mu| s\rangle\langle f \mid f\rangle=\langle p|\mu| s\rangle=\mu_{p s} \\
\left\langle s f|\mu| p_{1}^{\prime} f\right\rangle=\left\langle s|\mu| p_{1}^{\prime}\right\rangle\langle f \mid f\rangle=\left\langle s|\mu| p_{1}^{\prime}\right\rangle=\mu_{s p_{1}^{\prime}} \\
\left\langle s f|\mu| p_{3}^{\prime} f\right\rangle=\left\langle s|\mu| p_{3}^{\prime}\right\rangle\langle f \mid f\rangle=\left\langle s|\mu| p_{3}^{\prime}\right\rangle=\mu_{s p_{3}^{\prime}} \\
\left\langle p_{1}^{\prime} f|\mu| s^{\prime} f\right\rangle=\left\langle p_{1}^{\prime}|\mu| s^{\prime}\right\rangle\langle f \mid f\rangle=\left\langle p_{1}^{\prime}|\mu| s^{\prime}\right\rangle=\mu_{p_{1}^{\prime} s^{\prime}} \\
\left\langle p_{3}^{\prime} f|\mu| s^{\prime} f\right\rangle=\left\langle p_{3}^{\prime}|\mu| s^{\prime}\right\rangle\langle f \mid f\rangle=\left\langle p_{3}^{\prime}|\mu| s^{\prime}\right\rangle=\mu_{p_{3}^{\prime} s^{\prime}}
\end{gathered}
$$

At $R=\infty$ the frequencies of these transitions are equal to the atomic frequencies, and in each of these transitions one atom undergoes the transition while the other remains a spectator in the $(n-2) f$ state. These molecular matrix elements are reminiscent of isolated core excitation of the two electron Rydberg atoms. The frequencies relevant to the transitions shown in Fig. 4.1 are not near any atomic frequencies for either the atomic $f$ or $d$ states. For this reason the $(n-2) f$ atom is simply a spectator in the microwave transitions, and a $n d$ atom is unaffected by the microwave field.

The dipole-dipole interaction which is important for all transitions shown in Fig. 4.1 is

$$
V_{d d}=\left\langle n d_{5 / 2} n d_{5 / 2}\left|\frac{\mu \mu^{\prime}}{R^{3}}\right|(n+2) p_{3 / 2}(n-2) f\right\rangle
$$


where $\mu$ and $\mu^{\prime}$ are the dipole matrix elements of the two atoms, and $R$ is the distance between them. It can be written as

$$
\begin{aligned}
V_{d d} & =\frac{\left\langle n d_{5 / 2}|\mu|(n+2) p_{3 / 2}\right\rangle\left\langle n d_{5 / 2}\left|\mu^{\prime}\right|(n-2) f\right\rangle}{R^{3}} \\
& =\frac{\mu_{d p} \mu_{d f}}{R^{3}}
\end{aligned}
$$

Before we begin the description of the Floquet model for the transitions shown in Fig. 4.1, it is useful to summarize the CI model for the single photon $d d \rightarrow s f$ transition. Specifically, we are interested in calculating the fractional population transfer (FPT) from the $d d$ to the $s f$ state at resonance. To calculate the FPT from the $d d$ state at resonance we calculate the transition probability for a pair of atoms spaced by $R$ and then average over the spacings in the trap volume, as explained in some detail in Section 3.2.

From the six bare states shown in Fig. 4.1 we construct six Floquet states which are periodic, with the period of the microwave driving field. The Floquet energies are obtained by adding and subtracting integral multiples of the microwave frequency $\omega$ to the bare energies. We are interested in the Forster resonances shown in Fig. 4.1, which occur when the Floquet states based on the $s f, p_{1}^{\prime} f, p_{3}^{\prime} f$, and $s^{\prime} f$ states are degenerate with the $d d$ state. For $\mathrm{R}=\infty$ and zero microwave field these degeneracies occur when

$$
\begin{gathered}
W_{s f}-\omega=W_{d d} \\
W_{p_{1}^{\prime} f}-2 \omega=W_{d d} \\
W_{p_{3}^{\prime} f}-2 \omega=W_{d d} \\
W_{s^{\prime} f}-3 \omega=W_{d d}
\end{gathered}
$$




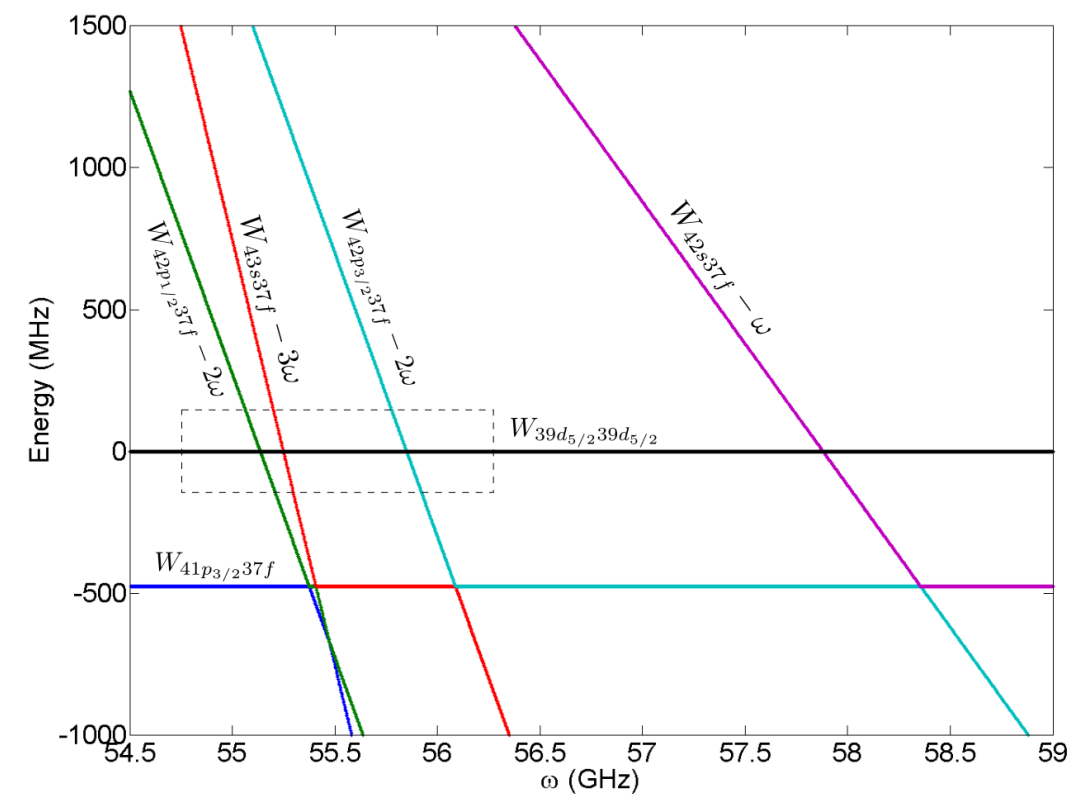

Figure 4.2: Floquet energy levels for $\mathrm{n}=39$ as a function of the microwave frequency for zero microwave field amplitude. No dipole-dipole interaction is included.

Accordingly, we restrict our attention to the Floquet energies $W_{d d}, W_{p f}, W_{s f}-\omega$, $W_{p_{1}^{\prime} f}-2 \omega, W_{p_{3}^{\prime} f}-2 \omega$, and $W_{s^{\prime} f}-3 \omega$. Ignoring Floquet energies in which other multiples of $\omega$ have been added or subtracted is equivalent to making the rotating wave approximation. Fig. 4.2 shows the Floquet energy levels for $n=39$ as a function of the microwave frequency for vanishing microwave field. The microwave resonances of Fig. 4.1 correspond to the level crossings of the $42 s 37 f, 42 p_{1 / 2} 37 f, 42 p_{3 / 2} 37 f$, and $43 s 37 f$ Floquet states with the $39 d_{5 / 2} 39 d_{5 / 2}$ state at frequencies $57.879,55.137$, 55.850, and 55.249 GHz. These are the Forster dipole-dipole energy transfer resonances from the $d d$ state to the Floquet states.

Equally important are the $42 p_{1 / 2} 37 f-43 s 37 f$ and $41 p_{3 / 2} 37 f-42 s 37 f$ crossings at 
55.473 and $58.356 \mathrm{GHz}$. These crossings are dipole allowed single photon microwave resonances, and in any finite field they become avoided crossings as can be seen in Fig. 4.3, altering all the energy levels but that of the $39 d_{5 / 2} 39 d_{5 / 2}$ state. These avoided crossings lead to AC Stark shifts of the Forster resonances.

In the presence of the linearly polarized microwave field $E \cos \omega t$ all the levels are coupled, by the matrix elements of Eq. (4.3), except $d d$, and the Floquet Hamiltonian matrix can be written as

$$
\mathcal{H}_{F}=\left(\begin{array}{cccccc}
W_{d d} & 0 & 0 & 0 & 0 & 0 \\
0 & W_{p f} & \mu_{s p} E / 2 & 0 & 0 & 0 \\
0 & \mu_{s p} E / 2 & W_{s f}-\omega & \mu_{p_{1}^{\prime} s} E / 2 & \mu_{p_{3}^{\prime} s} E / 2 & 0 \\
0 & 0 & \mu_{p_{1}^{\prime} s} E / 2 & W_{p_{1}^{\prime} f}-2 \omega & 0 & \mu_{s^{\prime} p_{1}^{\prime}} E / 2 \\
0 & 0 & \mu_{p_{3}^{\prime} s} E / 2 & 0 & W_{p_{3}^{\prime} f}-2 \omega & \mu_{s^{\prime} p_{3}^{\prime}} E / 2 \\
0 & 0 & 0 & \mu_{s^{\prime} p_{1}^{\prime}} E / 2 & \mu_{s^{\prime} p_{3}^{\prime}} E / 2 & W_{s^{\prime} f}-3 \omega
\end{array}\right)
$$

Diagonalizing this matrix yields the eigenvalues and eigenvectors. Since we have ignored the dipole-dipole interaction in this Floquet treatment, the energy $W_{d d}$ does not depend on the microwave field and one of the eigenstates is $|d d\rangle$. Each of the other five eigenstates we label as $\left|\psi_{F}\right\rangle$, where $\left|\psi_{F}\right\rangle$ is the linear combination

$$
\left|\psi_{F}\right\rangle=a_{1}|p f\rangle+a_{2}|s f\rangle+a_{3}\left|p_{1}^{\prime} f\right\rangle+a_{4}\left|p_{3}^{\prime} f\right\rangle+a_{5}\left|s^{\prime} f\right\rangle
$$

In Fig. 4.3 we show the Floquet energies over the same frequency range as shown in 


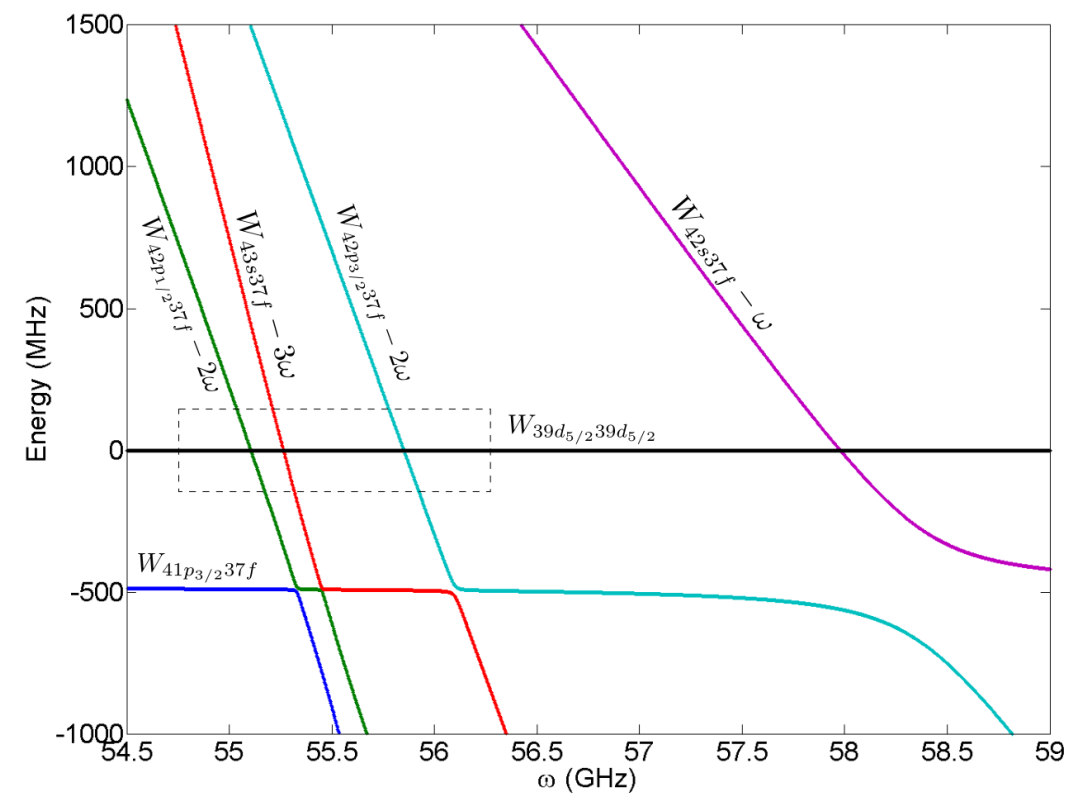

Figure 4.3: Floquet energy levels for $\mathrm{n}=39$ as a function of the microwave frequency for microwave field amplitude of $415 \mathrm{mV} / \mathrm{cm}$. No dipole-dipole interaction is included.

Fig. 4.2, but with a microwave field amplitude $E=415 \mathrm{mV} / \mathrm{cm}$.

The dipole moments of Eq. (4.7) are matrix elements of $z$, to correspond to the microwave polarization. We obtained the radial parts from Saffman and Walker[8] 
and the angular parts from Edmonds[1]. The specific values used are:

$$
\begin{aligned}
\mu_{s^{\prime} p_{3}^{\prime}} & =\frac{\sqrt{2}}{3}(1681) \\
\mu_{s^{\prime} p_{1}^{\prime}} & =-\frac{1}{3}(1650) \\
\mu_{p_{1}^{\prime} s} & =-\frac{1}{3}(1752) \\
\mu_{p_{3}^{\prime} s} & =\frac{\sqrt{2}}{3}(1728) \\
\mu_{s p} & =\frac{\sqrt{2}}{3}(1598)
\end{aligned}
$$

With the microwave field of $415 \mathrm{mV} / \mathrm{cm}$ the matrix element $\mu_{s p}$ leads to microwave coupling of $200 \mathrm{MHz}$, i.e. $\mu_{s p} E / 2=200 \mathrm{MHz}$.

Comparing Fig. 4.3 to Fig. 4.2, we can see that the single photon microwave resonances at 55.473 and $58.356 \mathrm{GHz}$ have become obvious avoided crossings, and the two photon $41 p_{3 / 2} 37 f-42 p_{j} 37 f$ resonances are visible. At the single microwave photon resonances the separation between Floquet levels, and the level shifts, are linear in the microwave field. Far from them, for example at the Forster level crossings with the $39 d_{5 / 2} 39 d_{5 / 2}$ state, the level shifts are quadratic in the microwave field. In Fig. 4.4 we show an expanded view of the portion of Fig. 4.3 containing the two and three photon Forster resonances (marked by a dashed box), in zero field and $\mathrm{E}=415$ $\mathrm{mV} / \mathrm{cm}$. At the frequency of the $39 d_{5 / 2} 39 d_{5 / 2}-42 p_{3 / 2} 37 f$ Forster resonance at 55.850 $\mathrm{GHz}$, the $42 p_{3 / 2} 37 f$ state lies about halfway between the $43 s_{1 / 2} 37 f$ and $42 s_{1 / 2} 37 f$ states, and the AC stark shifts due to these two states almost cancel, leading to a small AC stark shift of this Forster resonance. In contrast, at the frequency of the $39 d_{5 / 2} 39 d_{5 / 2}-42 p_{1 / 2} 37 f$ Forster resonance at $55.137 \mathrm{GHz}$, the $42 p_{1 / 2} 37 f$ state is 


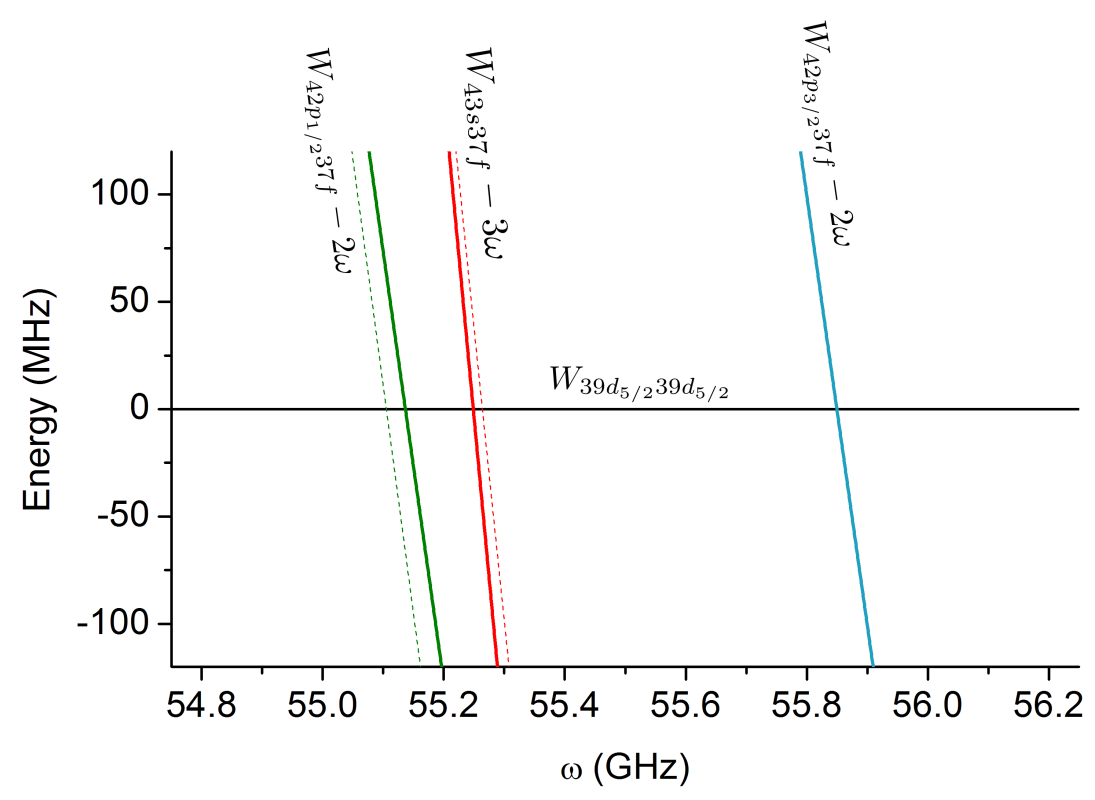

Figure 4.4: An expanded view of the region marked by dashed box in Fig. 4.2 and Fig. 4.3 containing the two and three photon Forster resonances. Solid lines and dotted lines represent microwave coupling of 0 and $200 \mathrm{MHz}$, respectively. For $W_{42 p_{3 / 2} 37 f}-2 \omega$, the solid line and the dotted line overlap. 
below both the $42 s_{1 / 2} 37 f$ and $43 s_{1 / 2} 37 f$ states, so the AC stark shifts add. More important, the Forster resonance is very close to the single photon $42 p_{1 / 2} 37 f-$ $43 s_{1 / 2} 37 f$ resonance, leading to a large frequency shift.

For small microwave fields the AC stark shifts of the Forster resonances are quadratic in the microwave field amplitude, and the calculated AC stark shifts are presented with the experimental results in the next section.

To calculate the fractional population transfer (FPT) from the laser excited $d d$ state to a Floquet state at a Forster resonance, we follow a procedure similar to that used in the development of Eqs. (3.9) to (3.12). We compute the transition probability for a pair of atoms spaced by distance $R$ and then average over the distribution of the spacings in the trap volume.

We calculate the transition probability at resonance for a transition from the $d d$ state to the Floquet state for a pair as follows. The microwave field is switched on and off in $10 \mathrm{~ns}$, which is fast compared to the dipole-dipole interaction. Thus, when the microwave field is switched on the population oscillates between $|d d\rangle$ and $\left|\psi_{F}\right\rangle$ at the frequency $\Omega$ given by the dipole-dipole coupling matrix element $\Omega=\left\langle d d\left|\frac{\mu \mu^{\prime}}{R^{3}}\right| \psi_{F}\right\rangle$. Since only $|p f\rangle$ of the $\left|\psi_{F}\right\rangle$ eigenfunction contributes to this matrix element,

$$
\Omega=\left\langle d d\left|\frac{\mu \mu^{\prime}}{R^{3}}\right| \psi_{F}\right\rangle=a_{1}\left\langle d d\left|\frac{\mu \mu^{\prime}}{R^{3}}\right| p f\right\rangle
$$

where $a_{1}$ is the coefficient given in Eq. (4.8). For $\Omega T>\pi$, on average half the oscillating population is in the $\psi_{F}$ state and is left there when the microwave field is turned off, in $10 \mathrm{~ns}$. As in the earlier development of Eqs. (3.9) to (3.12), the 
condition $\Omega T=\pi$ is met for $R=R_{T}$ where

$$
\Omega T=a_{1}\left\langle d d\left|\frac{\mu \mu^{\prime}}{R_{T}^{3}}\right| p f\right\rangle T=\pi
$$

For $R<R_{T}, \Omega T>\pi$, and the average transition probability is one half. For $R>R_{T}$ the transition probability falls rapidly with $R$. Accordingly, pairs with $R<R_{T}$ undergo the transition, and the FPT is again

$$
F P T=\frac{R_{T}^{3}}{R_{a v}^{3}}
$$

Where $R_{a v}$ is related to the density by

$$
\rho=\frac{3}{4 \pi R_{a v}^{3}}
$$

The population oscillation frequency $\Omega$ and the FPT are proportional to the Rydberg atom density and $a_{1}$, the coefficient of the $p f$ component of $\psi_{F}$. In the low microwave field limit, at each of the Forster resonances $a_{1}$ is proportional to $E^{M}$, where $M$ is the number of the photons absorbed or emitted.

To verify that the Floquet model gives the same result as the CI model discussed earlier, we restrict our attention to the one photon case treated using the CI model. For the one photon $d d-s f$ Forster resonance the microwave power is sufficiently low that only three states need be considered, $d d, p f$, and $s f$. In this case the only coupling in the Floquet matrix of Eq. (4.7) is that between the $p f$ and $s f$ states, 
and

$$
a_{1}=\frac{\mu_{p s} E}{2\left(W_{d d}-W_{p f}\right)} .
$$

Consequently,

$$
\Omega=\frac{\mu_{p s} \mu_{d p} \mu_{d f} E}{2 \Delta R^{3}},
$$

which is precisely the CI result of Eq. (3.9). In sum, the Floquet model allows us to predict the AC Stark shifts of the multiphoton resonances and the fractional population transfers, which scale as $E^{M}$, where $M$ is the number of microwave photons emitted or absorbed. Furthermore, the Floquet description is equivalent to the CI model presented previously to describe one photon transitions.

To compute the microwave fields required to observe the different transitions shown in Fig. 4.1, a useful criterion is the field required to produce a fixed value of $a_{1}$. In Table 4.1 we give the microwave fields required to produce $a_{1}=0.05$ for $\mathrm{n}=39$. As a comparison, the microwave field amplitude of $415 \mathrm{mV} / \mathrm{cm}$ was used to generate Fig. 4.3. From Table 4.1 it is apparent that similar microwave field strengths are required to observe the two photon transitions $d d \rightarrow p_{1}^{\prime} f$ and $d d \rightarrow p_{3}^{\prime} f$, since the detuning from the intermediate $s f$ state is large in both cases. However, the AC stark shift of the $d d \rightarrow p_{1}^{\prime} f$ resonance is much larger due to the proximity of the $d d \rightarrow p_{1}^{\prime} f$ Forster resonance to the single microwave photon $p_{1}^{\prime} f \rightarrow s^{\prime} f$ resonance.

\subsection{Experimental Approach}

The essential notion of the experiment can be understood with the aid of Fig. 4.5, which shows the relevant energy levels of single microwave photon transitions from 
Table 4.1: Microwave field amplitude required to produce $a_{1}=0.05$.

\begin{tabular}{cc} 
Transition & Required Microwave Field Amplitude $(\mathrm{mV} / \mathrm{cm})$ \\
\hline$n d_{5 / 2} n d_{5 / 2} \rightarrow(n+3) s_{1 / 2}(n-2) f_{7 / 2}$ & 49.8 \\
$n d_{5 / 2} n d_{5 / 2} \rightarrow(n+3) p_{1 / 2}(n-2) f_{7 / 2}$ & 622.5 \\
$n d_{5 / 2} n d_{5 / 2} \rightarrow(n+3) p_{3 / 2}(n-2) f_{7 / 2}$ & 456.5 \\
$n d_{5 / 2} n d_{5 / 2} \rightarrow(n+4) s_{1 / 2}(n-2) f_{7 / 2}$ & 1369.5
\end{tabular}

the $n d_{5 / 2} n d_{5 / 2}$ state. Pulsed laser excitation of atoms to the $n d_{5 / 2}$ state produces $n d_{5 / 2} n d_{5 / 2}$ pairs, which are coupled by the dipole-dipole interaction to the energetically nearby $(n+2) p_{3 / 2}(n-2) f$ state. A $1 \mu$ s long microwave pulse drives one of the four transitions, labelled A to D, in Fig. 4.5. The transitions are allowed due to the admixture of the $(n+2) p_{3 / 2}(n-2) f$ state into the $n d_{5 / 2} n d_{5 / 2}$ state by the dipole-dipole interaction. One of the atoms in the admixture interacts with the microwave field while the other remains a spectator. As shown by Fig. 4.5, which is approximately to scale, the microwave field can drive the pair to a lower or higher energy state. We detect that the pair has undergone the transition by applying a field ionization after the microwave pulse. We assume that the field ionization pulse projects the atoms onto isolated atomic states. For a transition to be observable one of the atoms in the final state pair must have an energy above the energy of the initially excited $n d$ state so that it is ionized earlier in the field ionization pulse. The $(n+2) p_{3 / 2},(n+1) d_{j}$, and $(n+3) s_{1 / 2}$ states meet this criterion. It is useful to note that in transition $\mathrm{D}$ although the microwave transition removes energy from the pair, the transition is detectable since the result is an $(n+2) p_{3 / 2}$ atom, which lies above the $n d$ state. The resonances corresponding to the transitions shown in Fig. 4.5 are recorded by setting the gate of a gated integrator on the signal due to 
field ionization of the $(n+2) p_{3 / 2},(n+1) d_{j}$, or $(n+3) s_{1 / 2}$ state and slowly sweeping the microwave frequency over many shots of the laser.

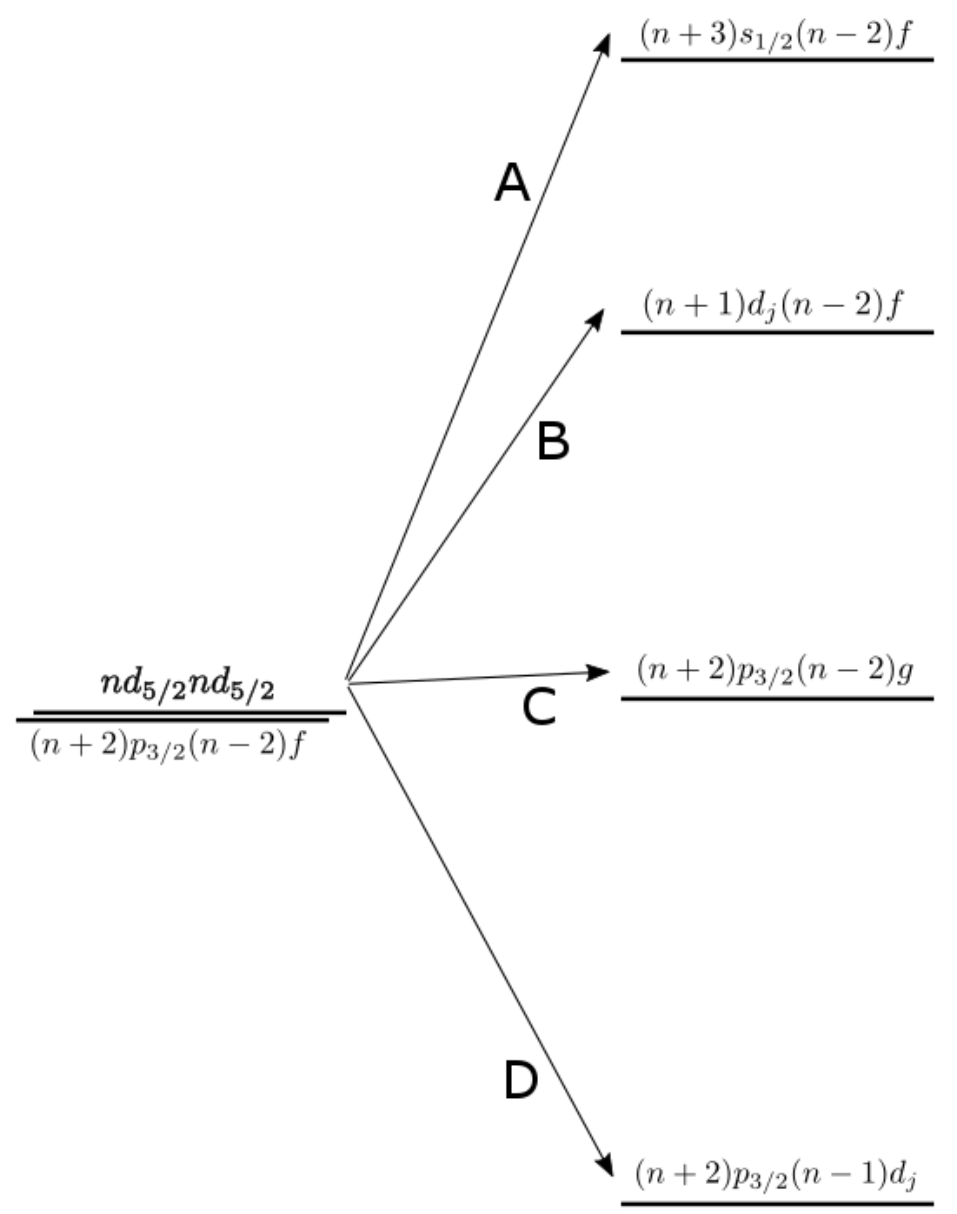

Figure 4.5: The observed one photon transitions originating from $n d_{5 / 2} n d_{5 / 2}$. The $(n+2) p_{3 / 2}(n-2) f$ state is nearly degenerate with $n d_{5 / 2} n d_{5 / 2}$ level. For $n=39$, $(n+2) p_{3 / 2}(n-2) f$ level is detuned by $477.8 \mathrm{MHz}$.

Since this experiment is an extension of the work described in Chapter 3, the 
experimental procedure is largely unchanged from the description in Section 3.3.

Some of the transitions require low frequencies, in the vicinity of $1 \mathrm{GHz}$. For those transitions, the output of the Agilent E8247C synthesizer is directly connected to the pair of rods closest to the MCP after going through Mini-circuits ZHL-42W amplifier and E\&M Labs L30Y circulator.

\subsection{Observations}

\section{One Photon Transitions from $n d_{5 / 2} n d_{5 / 2}$}

In Fig. 4.5 we show the single photon transitions from the $n d_{5 / 2} n d_{5 / 2}$ state. In all cases these transitions are possible due to the dipole-dipole induced admixture of the nearly degenerate $(n+2) p_{3 / 2}(n-2) f$ state into the $n d_{5 / 2} n d_{5 / 2}$ state.

The $n d_{5 / 2} n d_{5 / 2} \rightarrow(n+3) n s_{1 / 2}(n-2) f$ Transition (A)

Fig. 4.6 shows the observed $40 d_{5 / 2} 40 d_{5 / 2} \rightarrow 43 s_{1 / 2} 38 f_{7 / 2}$ resonances for a range of microwave field amplitudes. This transition corresponds to the transition A in Fig. 4.5 when $\mathrm{n}=40$. As the microwave field amplitude is raised, the transition exhibits $\mathrm{AC}$ Stark shifts to higher frequency. The AC Stark shift is caused by the fact that this transition is nearly resonant with the atomic $42 p_{3 / 2} \rightarrow 43 s_{1 / 2}$ transition. The relative microwave fields are determined from attenuator settings, whereas the absolute fields given in Fig. 4.6 are determined by calculating how much field is required to produce the observed shifts. In Table 4.2 we also present the AC Stark shifts calculated from our Floquet model as well as the maximum AC Stark shifts observed and the 


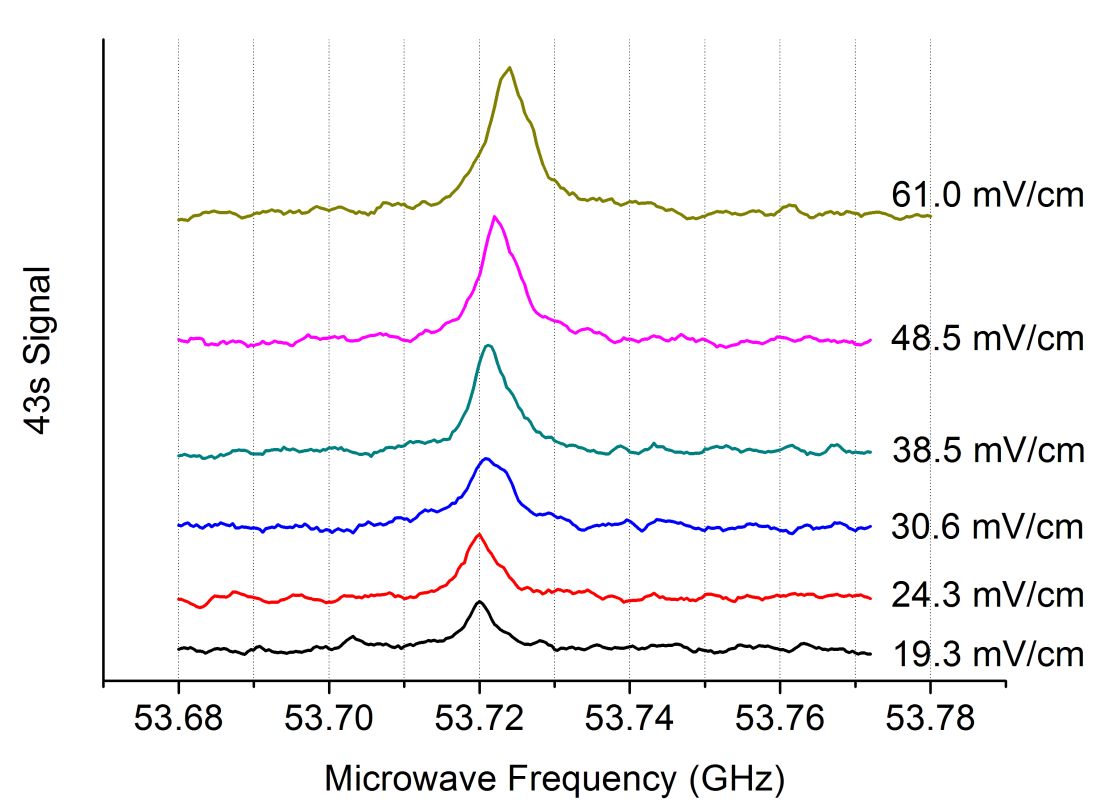

Figure 4.6: Observed $40 d_{5 / 2} 40 d_{5 / 2} \rightarrow 43 s_{1 / 2} 38 f_{7 / 2}$ resonances for a range of microwave field amplitudes. The calculated resonance frequency for the transition at zero microwave power and $R=\infty$ is $53.721 \mathrm{GHz}$.

estimated maximum field amplitude. The resonance frequency at zero microwave power is obtained by extrapolating the frequency of the resonance peak at different microwave field amplitudes back to zero power. The zero microwave power frequencies for our measurements are summarized in Table 4.2. Based on either the CI or Floquet model for a single photon transition, the fraction of atoms that is transferred to $(n+3) s_{1 / 2}(n-2) f$ state is expected to depend linearly on the microwave field amplitude. Fig. 4.7 shows the plot of FPT vs. the microwave field amplitude, exhibiting the expected linear behavior. 


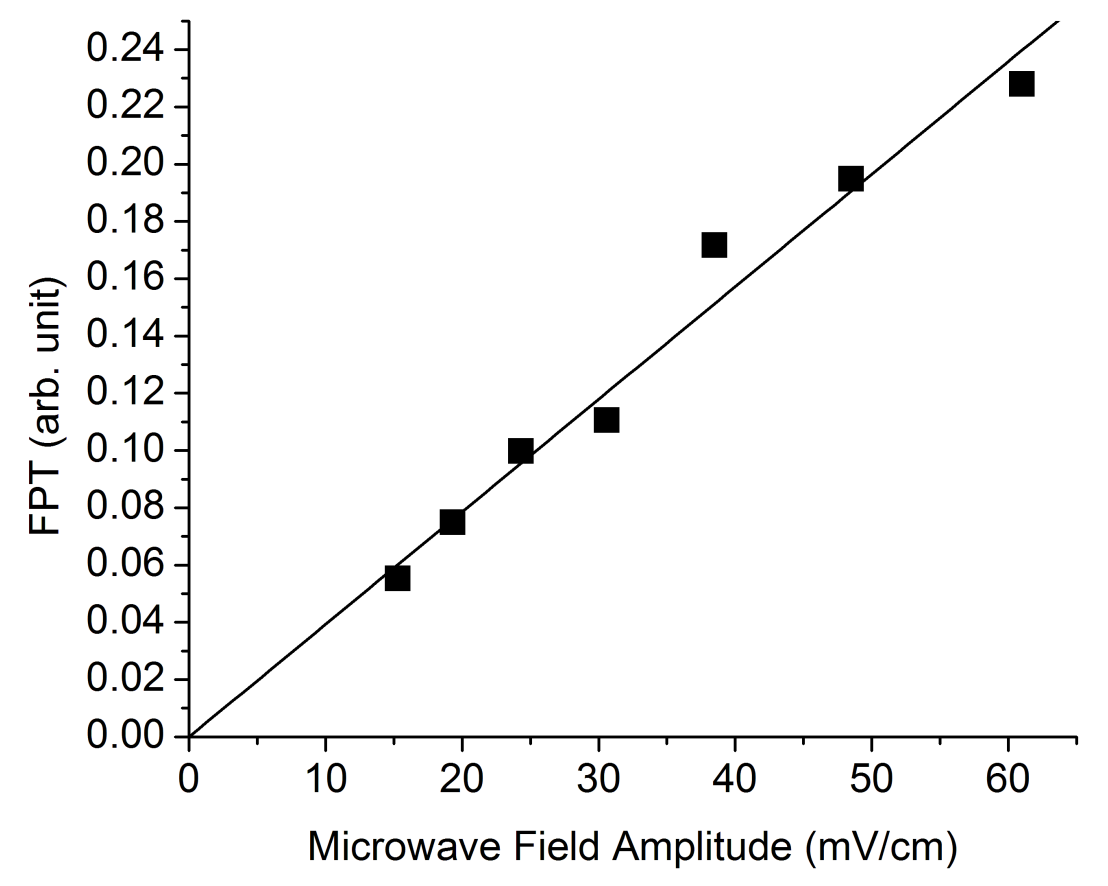

Figure 4.7: Fractional Population Transfer (FPT) vs. microwave field amplitude for the $40 d_{5 / 2} 40 d_{5 / 2} \rightarrow 43 s_{1 / 2} 38 f_{7 / 2}$ transition 
The $n d_{5 / 2} n d_{5 / 2} \rightarrow(n+1) d_{j}(n-2) f$ Transition (B)

Transition B in Fig. 4.5 was first reported by Yu et al. [9] in 2013. Further investigation of transition $\mathrm{B}$ as well as the observation of transition $\mathrm{D}$ were reported in the previous paper[2].

The $n d_{5 / 2} n d_{5 / 2} \rightarrow(n+2) p_{3 / 2}(n-2) g$ Transition (C)

Unlike transitions $\mathrm{A}$ and $\mathrm{B}$ of Fig. 4.5, in this case it is the $(n-2) f$ atom which undergoes the transition. Fig. 4.8 shows the resonant peak for the $41 d_{5 / 2} 41 d_{5 / 2} \rightarrow$ $43 p_{3 / 2} 39 g$ transition, which corresponds to transition C in Fig. 4.5. Due to the low frequency range required for the transition, the output of the microwave synthesizer is connected directly to the pair of rods closest to the MCP, as mentioned in the previous section. The transitions exhibit an AC Stark shift, linear in the radio frequency power, of up to $3 \mathrm{MHz}$, and the resonance frequencies at zero power are obtained by extrapolating to zero power. The results are summarized in Table 4.2. The ng-series quantum defect that is needed to calculate the intervals was taken from the paper by Lee et al.[3].

The $n d_{5 / 2} n d_{5 / 2} \rightarrow(n+2) p_{3 / 2}(n-1) d_{5 / 2}$ Transition (D)

There are two notable aspects to this transition. In addition to the $(n-2) f$ atom undergoing the transition, the transition is to a molecular state lower in energy than the $n d_{5 / 2} n d_{5 / 2}$ state. However, the atom left in the $(n+2) p_{3 / 2}$ state gains energy and can be distinguished from an $n d_{5 / 2}$ atom by field ionization. The observation of this transition has already been reported in Chapter 3. In Table 4.2, we report 


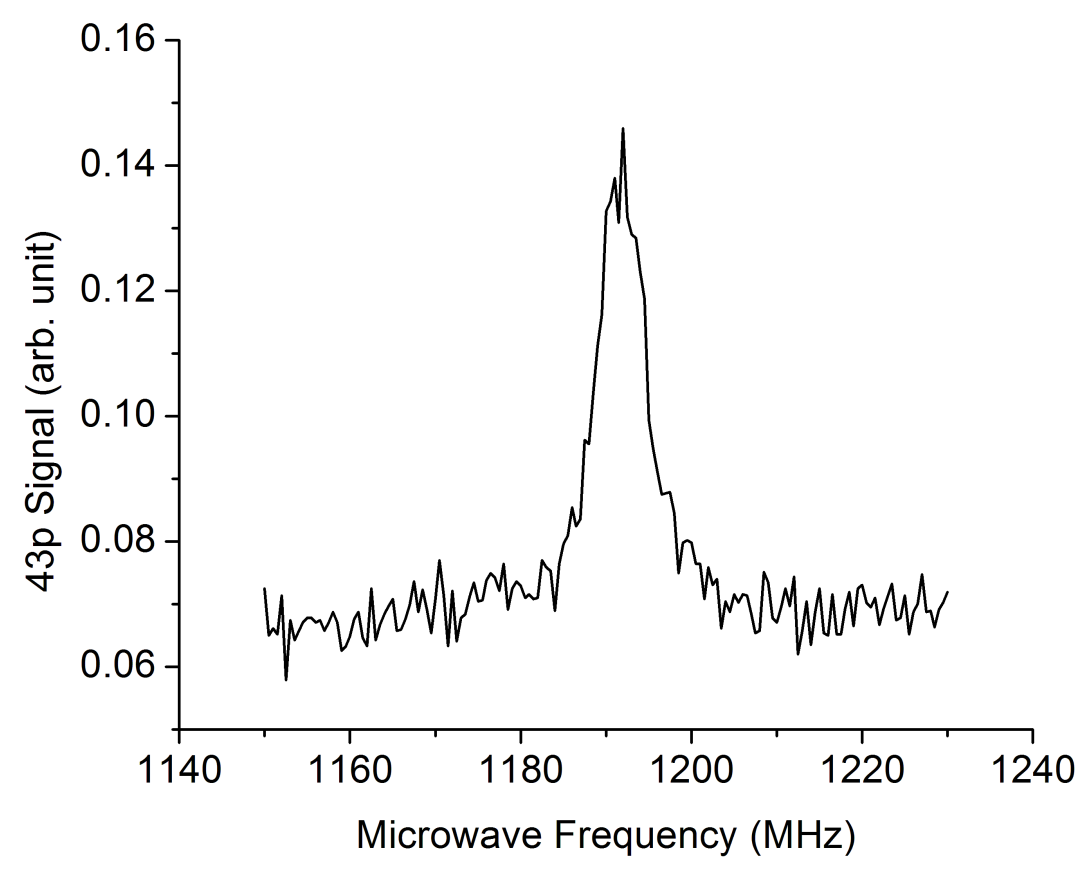

Figure 4.8: Observed resonance for the $41 d_{5 / 2} 41 d_{5 / 2} \rightarrow 43 p_{3 / 2} 39 g$ transition. The peak is shifted to higher frequency by $1.4 \mathrm{MHz}$ due to AC Stark shift. The calculated frequency for the transition is $1190.4 \mathrm{MHz}$.

more systematic measurements made to determine the transition frequencies at zero microwave power.

\section{One Photon Transitions from $n s_{1 / 2} n s_{1 / 2}$}

For the transitions originating from $n d_{5 / 2} n d_{5 / 2}$, it is the dipole-dipole induced configuration interaction with the nearby $(n+2) p_{3 / 2}(n-2) f$ state that allows the transitions. If we start with the $n s_{1 / 2} n s_{1 / 2}$ state, the nearest dipole-dipole coupled state $n p_{3 / 2}(n-1) p_{3 / 2}$ is much further away. As a concrete example, for $n=40$, 
Table 4.2: Resonance frequencies and AC Stark shift values for one photon transitions. Calculated shifts are obtained from our Floquet model. The estimated maximum field amplitudes are calculated from the maximum observed shifts and calculated shifts. As a point of comparison, the unattenuated microwave field amplitude with at the center of the trap is estimated to be $693 \mathrm{mV} / \mathrm{cm}$ by using the following parameters: Output power of the active doubler/quadrupler $=15 \mathrm{dBm}$, gain of the horn antenna $=20 \mathrm{dBi}$, distance from the horn to the center of trap $=$ $0.2 \mathrm{~m}$.

\begin{tabular}{|c|c|c|c|c|c|c|}
\hline Transition & $n$ & $\begin{array}{l}\text { Calculated } \\
(\mathrm{GHz})\end{array}$ & $\begin{array}{l}\text { Observed } \\
(\mathrm{GHz})\end{array}$ & $\begin{array}{l}\text { Calculated Shift } \\
\left(\mathrm{MHz} /(\mathrm{V} / \mathrm{cm})^{2}\right)\end{array}$ & $\begin{array}{l}\text { Max. Observed } \\
\text { Shift }(\mathrm{MHz})\end{array}$ & $\begin{array}{l}\text { Estimated Max. Field } \\
\text { Amplitude }(\mathrm{mV} / \mathrm{cm})\end{array}$ \\
\hline \multirow[t]{2}{*}{$n d_{5 / 2} n d_{5 / 2} \rightarrow(n+3) s_{1 / 2}(n-2) f_{7 / 2}$} & 39 & 57.879 & $57.876(9)$ & 486.8 & 18.4 & 194 \\
\hline & 40 & 53.721 & $57.718(11)$ & 791.1 & 73.4 & 305 \\
\hline \multirow{2}{*}{$n d_{5 / 2} n d_{5 / 2} \rightarrow(n+2) p_{3 / 2}(n-1) d_{5 / 2}$} & 39 & 43.921 & $43.923(8)$ & 348.4 & 29.4 & 290 \\
\hline & 40 & 40.415 & $40.417(8)$ & 570.6 & 20.1 & 188 \\
\hline \multirow[t]{2}{*}{$n d_{5 / 2} n d_{5 / 2} \rightarrow(n+2) p_{3 / 2}(n-1) d_{3 / 2}$} & 39 & 44.138 & $44.139(3)$ & 455.3 & 12.8 & 168 \\
\hline & 40 & 40.615 & $40.616(6)$ & 745.7 & 10.7 & 120 \\
\hline \multirow[t]{2}{*}{$n d_{5 / 2} n d_{5 / 2} \rightarrow(n+2) p_{3 / 2}(n-2) g$} & 41 & 1.190 & $1.190(11)$ & 130.9 & 3.3 & 159 \\
\hline & 42 & 1.193 & $1.190(5)$ & 305.2 & 3.6 & 109 \\
\hline \multirow[t]{2}{*}{$n s_{1 / 2} n s_{1 / 2} \rightarrow(n-1) d_{5 / 2}(n-1) p_{3 / 2}$} & 39 & 34.010 & $34.010(9)$ & 36.7 & $<1$ & \\
\hline & 40 & 31.441 & $31.441(10)$ & 44.9 & $<1$ & \\
\hline
\end{tabular}

$\Delta_{40 s_{1 / 2} 40 s_{1 / 2}-40 p_{3 / 2} 39 p_{3 / 2}}=5.45 \mathrm{GHz}$, whereas $\Delta_{40 d_{5 / 2} 40 d_{5 / 2}-42 p_{3 / 2} 38 f_{7 / 2}}=325 \mathrm{MHz}$. The large detuning for $n s_{1 / 2} n s_{1 / 2}-n p_{3 / 2}(n-1) p_{3 / 2}$ results in a small admixture coefficient. Nonetheless, it is possible to observe transitions similar to the observed transitions originating from $n d_{5 / 2} n d_{5 / 2}$.

The $n s_{1 / 2} n s_{1 / 2} \rightarrow(n-1) d_{5 / 2}(n-1) p_{3 / 2}$ Transition

Fig. 4.9 shows the energy levels involved in $n s_{1 / 2} n s_{1 / 2} \rightarrow(n-1) d_{5 / 2}(n-1) p_{3 / 2}$ transition which is one of the possible transitions originating from $n s_{1 / 2} n s_{1 / 2}$. The diagram is approximately to scale, and the large detuning between $n s_{1 / 2} n s_{1 / 2}$ and $n p_{3 / 2}(n-1) p_{3 / 2}$ is evident. Fig. 4.10 shows the observed resonance for $n=40$. The resonant peak does not observably shift when the microwave power is raised because the microwave frequency is not near the $40 p_{3 / 2} \rightarrow 39 d_{5 / 2}$ resonant frequency. 


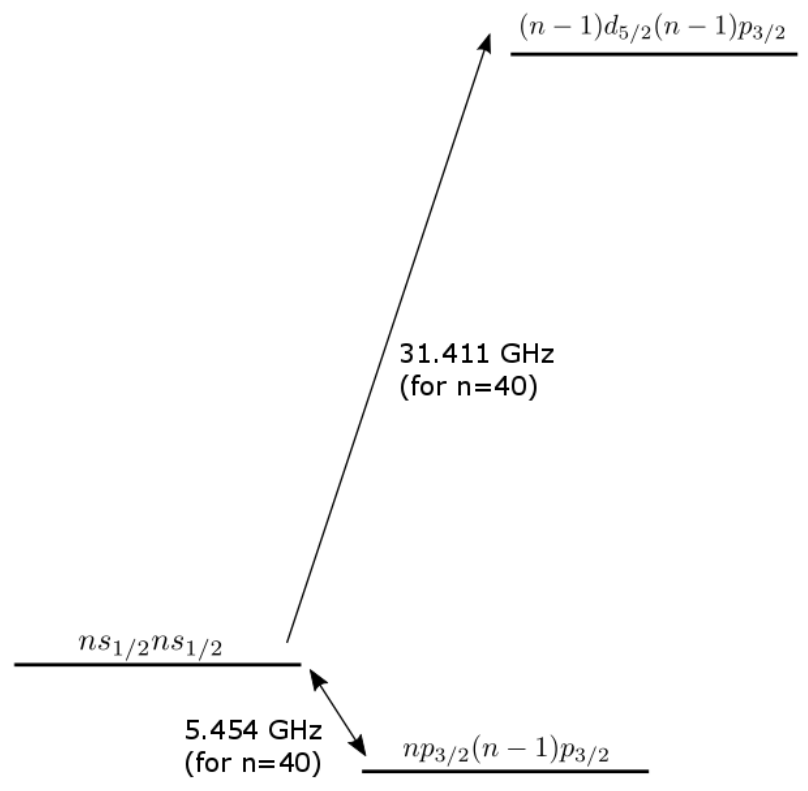

Figure 4.9: One photon transition originating from $n s_{1 / 2} n s_{1 / 2}$ that was observed. 


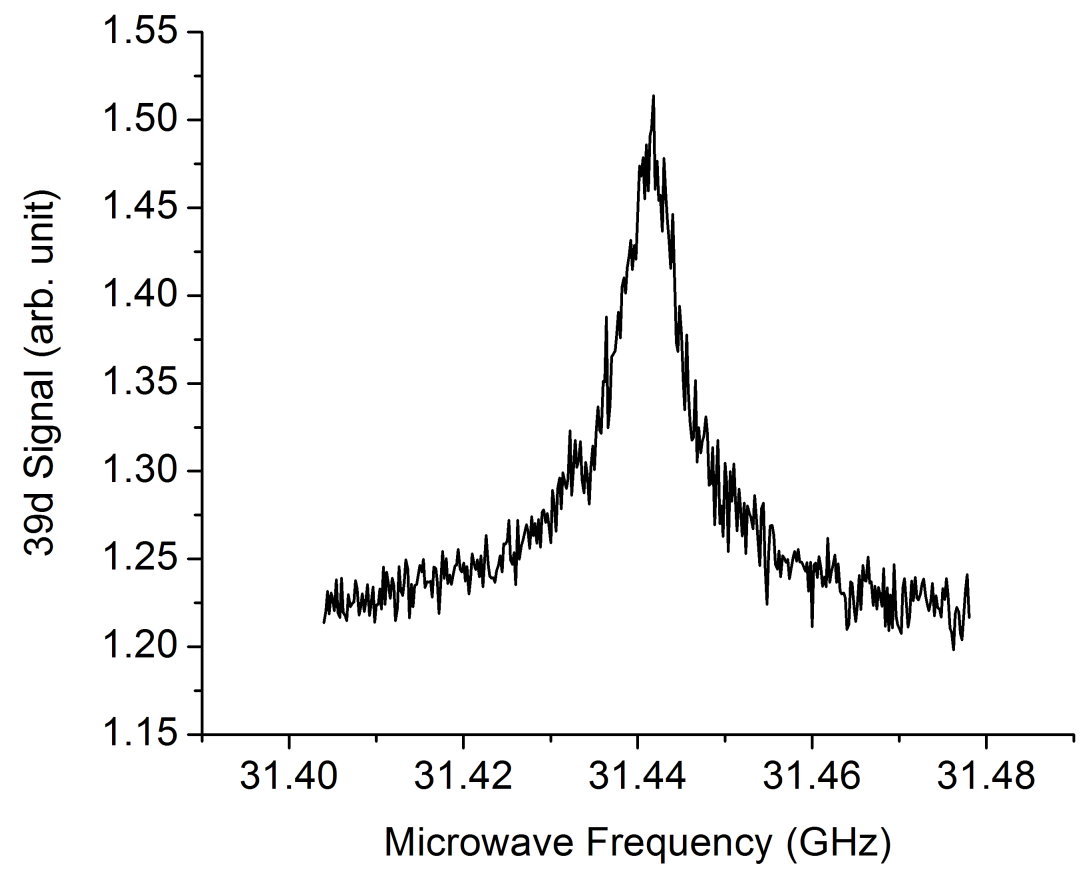

Figure 4.10: Observed resonance for $40 s_{1 / 2} 40 s_{1 / 2} \rightarrow 39 d_{5 / 2} 39 p_{3 / 2}$ transition. The calculated frequency for the transition is $31.441 \mathrm{GHz}$. The peak is not observably shifted. 


\section{Multiphoton Transitions from $n d_{5 / 2} n d_{5 / 2}$}

In addition to the transitions discussed so far, transitions that involve more than one microwave photon have been observed. Fig. 4.1 shows the observed single and multi photon transitions originating from $n d_{5 / 2} n d_{5 / 2}$ state. Observing the multiphoton transitions requires higher microwave field amplitude.

The $n d_{5 / 2} n d_{5 / 2} \rightarrow(n+3) p_{1 / 2}(n-2) f$ Transition

Fig. 4.11 shows the observed $39 d_{5 / 2} 39 d_{5 / 2} \rightarrow 42 p_{1 / 2} 37 f_{7 / 2}$ resonances for a range of microwave field amplitudes. This is a two photon transition, and the resonances exhibit a large AC Stark shift to lower frequency. The microwave field amplitudes involved here are greater than those in Fig. 4.6 by more than an order of magnitude. The microwave field amplitudes were estimated from the observed shifts. As discussed earlier, for a two photon transition, the fraction of atoms that is transferred to $(n+3) p_{j}(n-2) f$ state is expected to scale as the square of the microwave field amplitude, or linearly in the microwave power. Fig. 4.12 shows the plot of FPT vs. microwave field amplitude squared and exhibits the expected scaling. The obtained zero power frequencies are given in Table 4.3.

The $n d_{5 / 2} n d_{5 / 2} \rightarrow(n+3) p_{3 / 2}(n-2) f$ Transition

Fig. 4.13 shows the observed $39 d_{5 / 2} 39 d_{5 / 2} \rightarrow 42 p_{3 / 2} 37 f_{7 / 2}$ resonances for a range of microwave field amplitudes. Although the frequency for this transition lies near the $n d_{5 / 2} n d_{5 / 2} \rightarrow(n+3) p_{1 / 2}(n-2) f$ transition frequency, there is an important difference: The resonant peak for this transition does not observably shift when the 


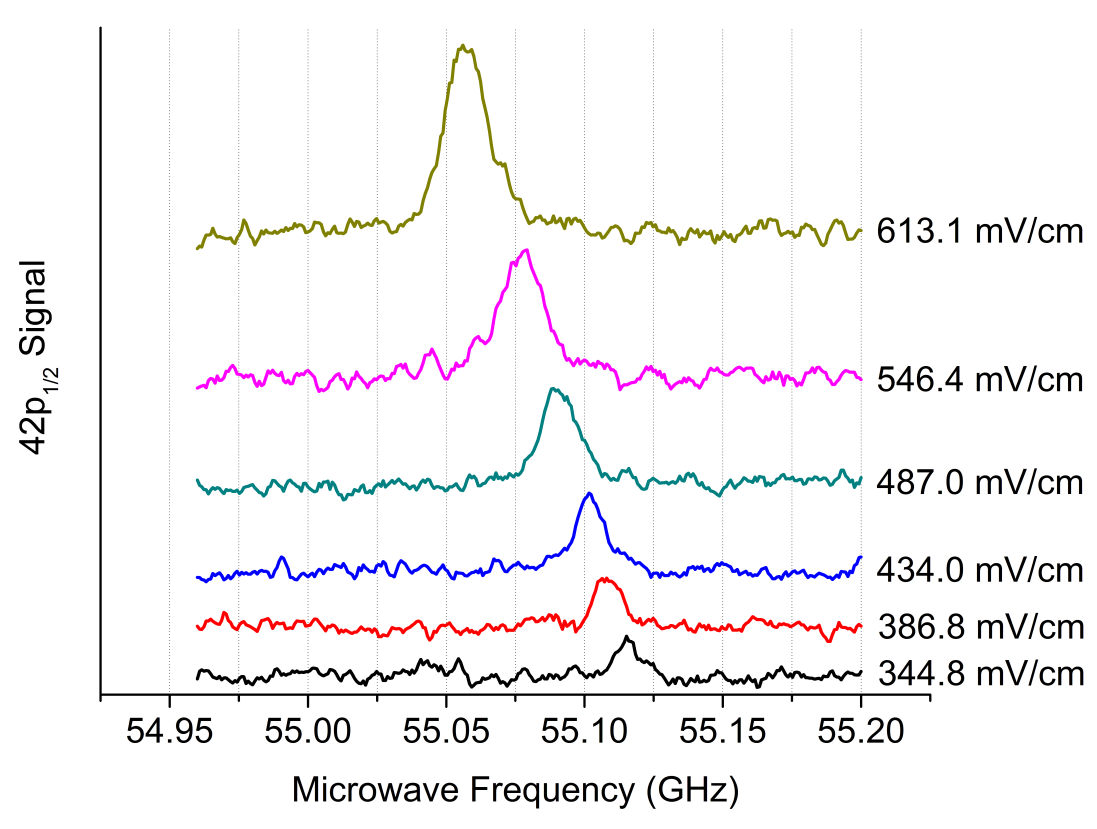

Figure 4.11: Observed $39 d_{5 / 2} 39 d_{5 / 2} \rightarrow 42 p_{1 / 2} 37 f_{7 / 2}$ resonances for a range of microwave field amplitudes. The calculated two photon resonance frequency for the transition at zero microwave power and $R=\infty$ is $110.273 \mathrm{GHz}$. 


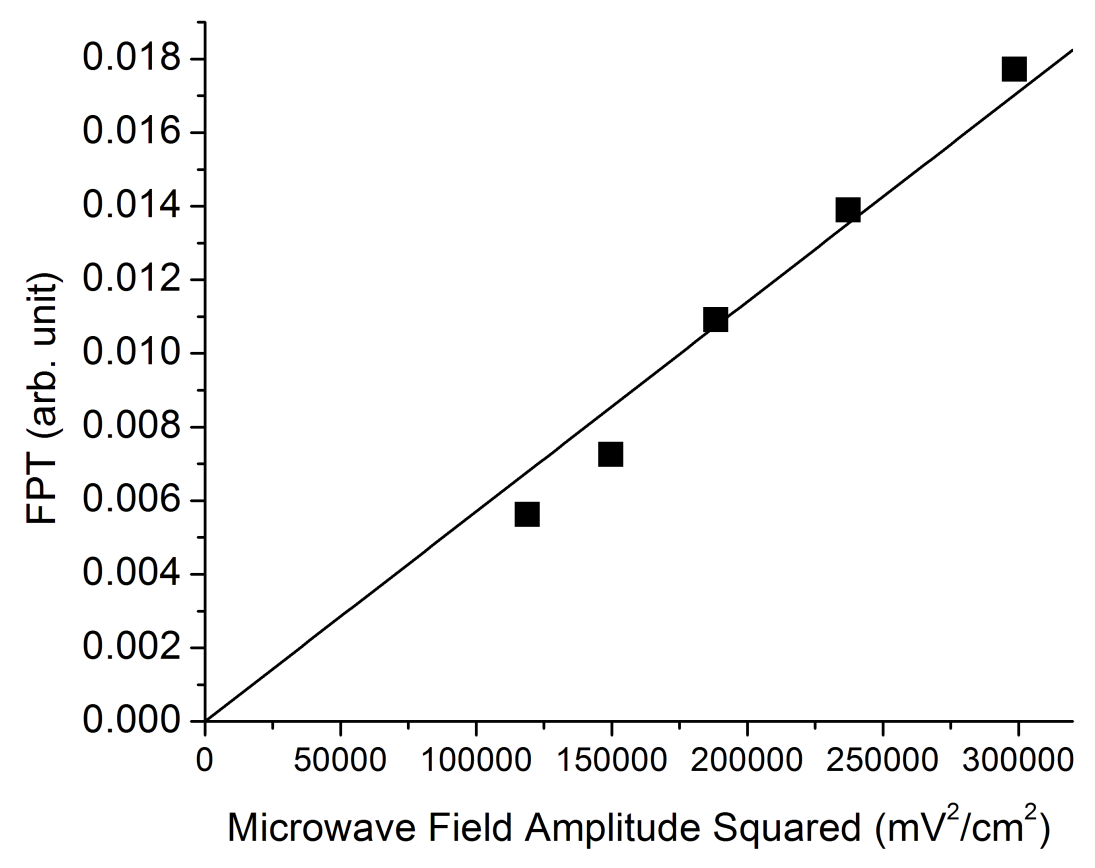

Figure 4.12: Fractional Population Transfer (FPT) vs. microwave field amplitude squared for the $39 d_{5 / 2} 39 d_{5 / 2} \rightarrow 42 p_{1 / 2} 37 f_{7 / 2}$ transition 


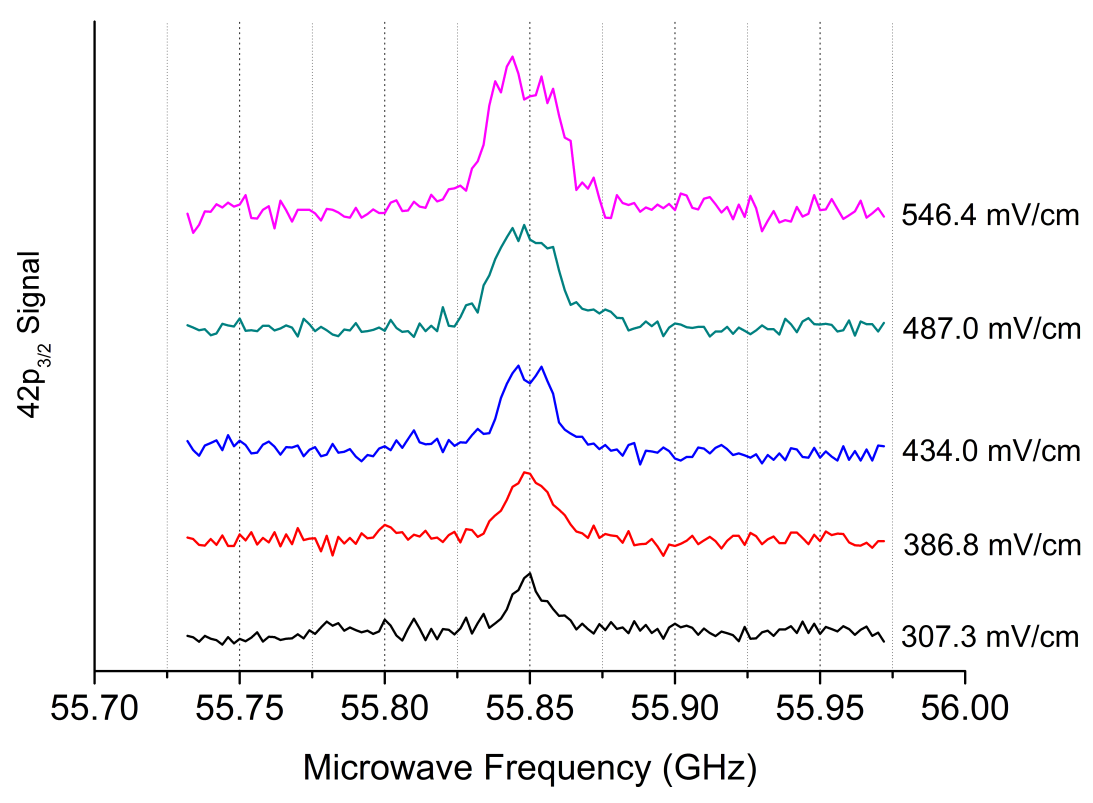

Figure 4.13: Observed $39 d_{5 / 2} 39 d_{5 / 2} \rightarrow 42 p_{3 / 2} 37 f_{7 / 2}$ resonances for a range of microwave field amplitudes. The calculated two photon resonance frequency for the transition at zero microwave power and $R=\infty$ is $111.699 \mathrm{GHz}$. 
Table 4.3: Resonance frequencies and AC Stark shift values for two photon transitions. Calculated shifts are obtained from our Floquet model. The estimated maximum field amplitudes are calculated from the maximum observed shifts and calculated shifts. As a point of comparison, the unattenuated microwave field amplitude with at the center of the trap is estimated to be $693 \mathrm{mV} / \mathrm{cm}$ by using the following parameters: Output power of the active doubler/quadrupler $=15 \mathrm{dBm}$, gain of the horn antenna $=20 \mathrm{dBi}$, distance from the horn to the center of trap $=$ $0.2 \mathrm{~m}$.

\begin{tabular}{ccccccc} 
Transition & $n$ & $\begin{array}{c}\text { Calculated } \\
(\mathrm{GHz})\end{array}$ & $\begin{array}{c}\text { Observed } \\
(\mathrm{GHz})\end{array}$ & $\begin{array}{c}\text { Calculated Shift } \\
\left(\mathrm{MHz} /(\mathrm{V} / \mathrm{cm})^{2}\right)\end{array}$ & $\begin{array}{c}\text { Max. Observed } \\
\text { Shift }(\mathrm{MHz})\end{array}$ & $\begin{array}{c}\text { Estimated Max. Field } \\
\text { Amplitude }(\mathrm{mV} / \mathrm{cm})\end{array}$ \\
\hline$n d_{5 / 2} n d_{5 / 2} \rightarrow(n+3) p_{1 / 2}(n-2) f_{7 / 2}$ & 39 & 110.273 & $110.280(51)$ & 419.9 & 118.6 & \\
& 40 & 102.294 & $102.294(31)$ & 482.1 & 50.8 & 31 \\
$n d_{5 / 2} n d_{5 / 2} \rightarrow(n+3) p_{3 / 2}(n-2) f_{7 / 2}$ & 39 & 111.699 & $111.697(18)$ & 8.6 & $<1$ & 325 \\
& 40 & 103.616 & $103.615(10)$ & 15.5 & $<1$
\end{tabular}

microwave power is raised. As discussed earlier, the suppression of the AC Stark shift is caused by the fact that the $(n+3) p_{3 / 2}$ state lies approximately halfway between the $(n+3) s_{1 / 2}$ and $(n+4) s_{1 / 2}$ states. As a result, the AC Stark shift contributions due to the $(n+3) s_{1 / 2}$ and $(n+4) s_{1 / 2}$ states nearly cancel. The calculated AC Stark shifts are given in Table 4.3 as well as the observed and calculated zero power frequencies.

The $n d_{5 / 2} n d_{5 / 2} \rightarrow(n+4) s_{1 / 2}(n-2) f$ Transition

Fig. 4.14 shows the observed resonance for the $40 d_{5 / 2} 40 d_{5 / 2} \rightarrow 44 s_{1 / 2} 38 f$ transition. The peak is shifted to higher frequency by $25 \mathrm{MHz}$ due to the AC Stark shift. This three-photon transition requires a high microwave field amplitude, and the resonance peaks can only be obtained for the microwave field amplitudes close to the maximum possible value. The calculated AC Stark shifts are given in Table 4.4 as well as the observed and calculated zero power frequencies. 


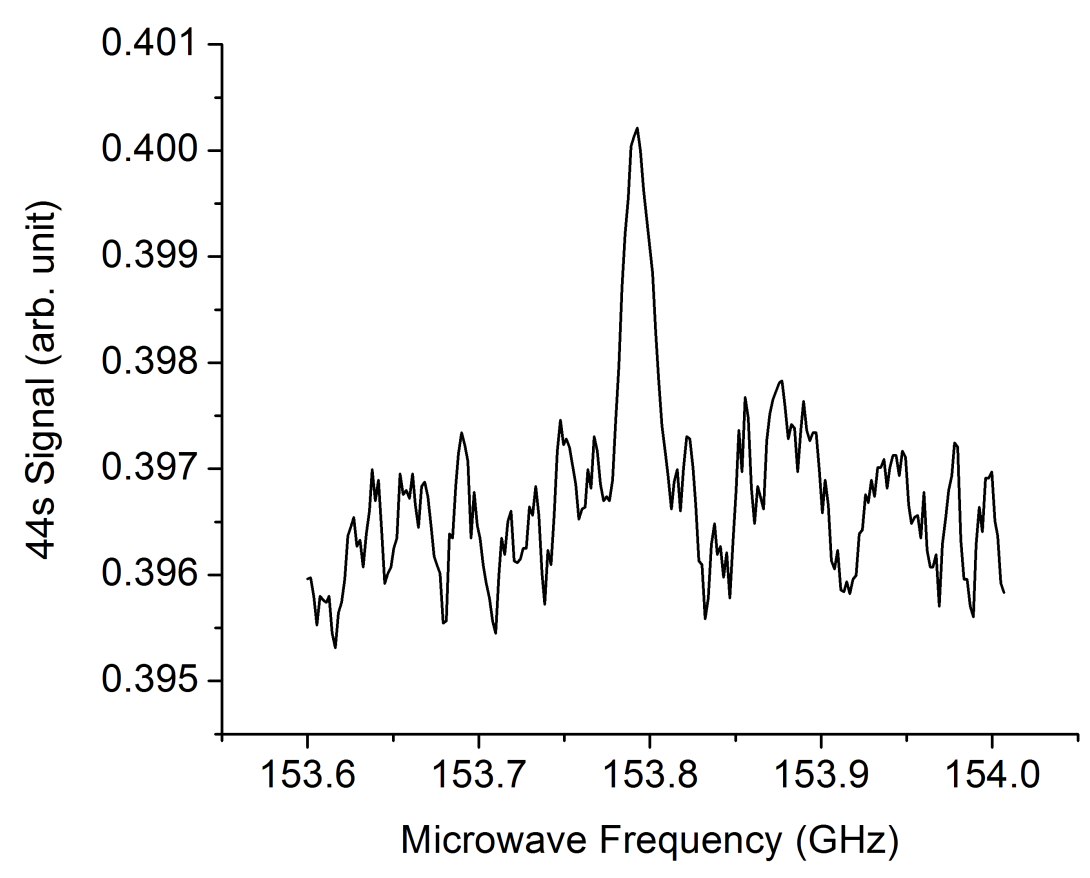

Figure 4.14: The observed resonance for $40 d_{5 / 2} 40 d_{5 / 2} \rightarrow 44 s_{1 / 2} 38 f$ transition. The peak is shifted to higher frequency by $25 \mathrm{MHz}$ due to AC Stark shift.

Table 4.4: Resonance frequencies and AC Stark shift values for three photon transition. Calculated shifts are obtained from our Floquet model. The estimated maximum field amplitudes are calculated from the maximum observed shifts and calculated shifts. As a point of comparison, the unattenuated microwave field amplitude with at the center of the trap is estimated to be $693 \mathrm{mV} / \mathrm{cm}$ by using the following parameters: Output power of the active doubler/quadrupler $=15 \mathrm{dBm}$, gain of the horn antenna $=20 \mathrm{dBi}$, distance from the horn to the center of trap $=0.2 \mathrm{~m}$.

\begin{tabular}{ccccccc} 
Transition & $n$ & $\begin{array}{c}\text { Calculated } \\
(\mathrm{GHz})\end{array}$ & $\begin{array}{c}\text { Observed } \\
(\mathrm{GHz})\end{array}$ & $\begin{array}{c}\text { Calculated Shift } \\
\left(\mathrm{MHz} /(\mathrm{V} / \mathrm{cm})^{2}\right)\end{array}$ & $\begin{array}{c}\text { Max. Observed } \\
\text { Shift }(\mathrm{MHz})\end{array}$ & $\begin{array}{c}\text { Estimated Max. Field } \\
\text { Amplitude }(\mathrm{mV} / \mathrm{cm})\end{array}$ \\
\hline$n d_{5 / 2} n d_{5 / 2} \rightarrow(n+4) s_{1 / 2}(n-2) f_{7 / 2}$ & 40 & 153.768 & $153.767(79)$ & 87.2 & 40.2 & 679
\end{tabular}




\subsection{Conclusion}

These measurements show that it is straightforward to drive microwave transitions between pairs of atoms even when the dipole-dipole detuning is large, $\sim 5 \mathrm{GHz}$. Both single photon and multiphoton transitions can be described as Forster resonances of Floquet states tuned into resonance with the microwave frequency. While the Floquet approach is particularly convenient for multiphoton transitions as it is easily extended to stronger fields, it reduces to the CI approach used previously for single photon transitions. 


\section{REFERENCES}

[1] Edmonds, A. (1960). Angular Momentum in Quantum Mechanics. Princeton, NJ: Princeton University Press.

[2] Lee, J. \& Gallagher, T. F. (2016). Microwave transitions from pairs of rb $n d_{5 / 2} n d_{5 / 2}$ atoms. Phys. Rev. A, 93, 062509.

[3] Lee, J., Nunkaew, J., \& Gallagher, T. F. (2016). Microwave spectroscopy of the cold rubidium $(n+1) d_{5 / 2} \rightarrow n g$ and $n h$ transitions. Phys. Rev. A, 94, 022505.

[4] Pillet, P., Kachru, R., Tran, N. H., Smith, W. W., \& Gallagher, T. F. (1987). Radiative rydberg-atom ${ }^{`}$ rydberg-atom collisions in the strong-field regime. Phys. Rev. A, 36, 1132-1147.

[5] Shirley, J. H. (1965). Solution of the schrödinger equation with a hamiltonian periodic in time. Phys. Rev., 138, B979-B987.

[6] Teixeira, R. C., Hermann-Avigliano, C., Nguyen, T. L., Cantat-Moltrecht, T., Raimond, J. M., Haroche, S., Gleyzes, S., \& Brune, M. (2015). Microwaves probe dipole blockade and van der waals forces in a cold rydberg gas. Phys. Rev. Lett., $115,013001$.

[7] van Ditzhuijzen, C. S. E., Koenderink, A. F., Hernández, J. V., Robicheaux, F., Noordam, L. D., \& van den Heuvell, H. B. v. L. (2008). Spatially resolved observation of dipole-dipole interaction between rydberg atoms. Phys. Rev. Lett., $100,243201$.

[8] Walker, T. G. \& Saffman, M. (2008). Consequences of zeeman degeneracy for the van der waals blockade between rydberg atoms. Phys. Rev. A, 77, 032723.

[9] Yu, Y., Park, H., \& Gallagher, T. F. (2013). Microwave transitions in pairs of rb rydberg atoms. Phys. Rev. Lett., 111, 173001. 


\section{Chapter 5}

\section{Quantum Defects and Ionic Dipole and Quadrupole Polarizabilities}

\subsection{Introduction}

Precise values for the quantum defects of the high angular momentum states are important in the calculations of the Stark effect, which are particularly important for Förster resonant dipole-dipole energy transfer involving Rydberg atoms [4, 25, 29]. Moreover, the ionic dipole and quadrupole polarizabilities of atoms can be determined from the same quantum defects since they arise from polarization of the core [22]. The dipole polarizabilities of alkaline earth ions are of interest for clock applications, and the dipole polarizabilities of alkali atoms are of interest as benchmarks for atomic structure calculations relevant to parity violation measurements and atom interferometry $[6,10,12,14,23,24]$. While most of the polarizability of the ground 
state of an alkali atom is due to the valence electron, the contribution of the ionic core is not insignificant. For example, the $\mathrm{Rb}^{+}$dipole polarizability represents $3 \%$ of the $\mathrm{Rb} 5 s$ ground state polarizability [31]. For this reason, it is important to measure the dipole polarizabilities of alkali ions.

Previous experimental values for the $\mathrm{Rb}^{+}$dipole and quadrupole polarizabilities were determined from the $\mathrm{Rb} n f$ and $n g$ quantum defects in spite of the inverted fine structure of the $n f$ states $[13,17]$, the typical signature of the highest $\ell$ core penetrating state [33]. Here $\ell$ is the orbital angular momentum of the Rydberg electron. A third measurement used only the $n f$ series, and it is difficult to extract the polarizabilities from one $\ell$ series [16]. In all cases, the residual core penetration of the $n f$ series leads to a large uncertainty in the $\mathrm{Rb}^{+}$dipole and quadrupole polarizabilities in previous work $[13,16,17]$. To obtain better values for the core polarizabilities it would be desirable to measure the quantum defects of non penetrating $\ell>3$ states. However, as $\ell$ is increased the intervals between the $\ell$ states decrease, and the Stark shifts due to small stray fields become a significant problem. To observe the intervals in zero field the field must be nulled in all directions. However, it is often the case that in an existing apparatus the field can only be nulled in one direction, leaving an unknown field in the plane perpendicular to that direction, and an unknown frequency shift.

Here we report the use of an experimental technique to determine zero field intervals in spite of the fact that we can only null the field in one direction. Specifically, we measure a low field parameter which depends on the static field $E_{S}$, not its square $E_{S}^{2}$. This approach enables us to determine the remaining perpendicular field and 
extrapolate the observed frequencies to zero field. We have used this approach to measure the $\mathrm{Rb}$ zero field $(n+1) d_{5 / 2} \rightarrow n g$ and $n h$ intervals. We are not able to resolve the $n g$ and $n h$ fine structure intervals. Based on the $\ell$ dependence of the fine structure intervals in other alkali atoms, we expect them to be close to the hydrogenic values. For example, the $28 g$ and $28 h$ intervals are $0.40 \mathrm{MHz}$ and $0.27 \mathrm{MHz}$ for hydrogen $[5,8,11,35]$. Combining these intervals with the known $\mathrm{Rb}$ nd quantum defects we derive the quantum defects of the $\mathrm{Rb} n g$ and $n h$ states of $27 \leq n \leq 30$. From these quantum defects we extract substantially improved values for the $\mathrm{Rb}^{+}$ ionic dipole and quadrupole polarizabilities. In the sections which follow we describe the principle of the approach, the experimental method, our observations, and the core polarization analysis.

\subsection{Principle}

To illustrate the principle of the approach, as an example we describe extracting the zero field $(n+1) d_{5 / 2} \rightarrow n g$ intervals. The Stark shifts of the levels and the frequency shift of the $(n+1) d_{5 / 2} \rightarrow n g$ transition are proportional to $E_{S}^{2}$ the squared magnitude of the static field $E_{S}$. It is convenient to write $E_{S}^{2}$ as

$$
E_{S}^{2}=E_{z}^{2}+E_{\perp}^{2},
$$

where the field $\vec{E}_{\perp}$ lies in the plane perpendicular to the $z$ direction. The frequency $\nu_{d_{5 / 2} \rightarrow g}$ of the $(n+1) d_{5 / 2} \rightarrow n g$ transition is given by 


$$
\nu_{d_{5 / 2} \rightarrow g}=\nu_{0, d_{5 / 2} \rightarrow g}+P E_{S}^{2},
$$

where $P$ is half the difference in the polarizabilities of the $d$ and $g$ states, and $\nu_{0, d_{5 / 2} \rightarrow g}$ is the zero field interval. The direction of $\vec{E}_{S}$ is unimportant. By applying the bias voltage $V_{b}$ we are able to control the static field in the $z$ direction $\left(E_{z}\right)$, and if we measure the resonance frequency as a function of bias voltage $V_{b}$, or bias field $E_{b}$, we observe a parabola, with the maximum frequency $\nu_{0, d_{5 / 2} \rightarrow g}+P E_{\perp}^{2}$. This procedure leaves us with an unknown frequency shift of $P E_{\perp}^{2}$ because we cannot extrapolate $E_{S}^{2}$ to zero if only $E_{z}$ is altered, as shown by Eq. (5.1).

In contrast, if we measure a property $X$ which is simply related to $E_{S}$, given explicitly by

$$
E_{S}=\sqrt{E_{z}^{2}+E_{\perp}^{2}},
$$

we can extrapolate to $E_{S}=0$ and determine $E_{\perp}^{2}$. As an example, we consider the case in which $X$ is proportional to $E_{S}$. The procedure is to measure the resonance frequency $\nu_{d_{5 / 2} \rightarrow g}$ and $X$ as functions of $V_{b}$, or $E_{b}$, and plot $\nu_{d_{5 / 2} \rightarrow g}$ vs $X^{2}$. The observed frequencies should fall on a straight line, as shown by Eq. (5.2), and the $X^{2}=0$ intercept is the zero field $(n+1) d_{5 / 2} \rightarrow n g$ interval.

The challenge is to identify the appropriate property $X$, and we have explored two different ones. In the first approach, $X$ is the separation between Stark states. We take advantage of the fact that, for the high $\ell$ states, the quantum defects are very small. Therefore, the zero field $\ell$ states are converted to Stark $k$ states, which exhibit linear Stark shifts, even in very small electric fields. A Stark state is assigned 
the label $k$, equal to the $\ell$ of the zero field state to which it is adiabatically connected. The separation between adjacent Stark states, $\Delta \nu_{S}=3 n E_{S}$, is linear in the field $E_{S}$ [2]. We observe the microwave transitions from the $(n+1) d$ states to the $n k$ Stark states and implicitly determine $E_{S}$ from $\Delta \nu_{S}$, the separation between adjacent Stark states. For each bias voltage we obtain the value of $\Delta \nu_{S}$ from the Stark spectrum, and we measure the $(n+1) d_{5 / 2} \rightarrow n g$ resonance frequency $\nu_{d_{5 / 2} \rightarrow g}$. Plotting the measured frequency $\nu_{d_{5 / 2} \rightarrow g}$ vs $\Delta \nu_{S}^{2}$ allows extrapolation to the zero field interval, as shown by Eq. (5.2). An attractive feature of this approach is that only frequencies are measured.

In the second approach, $X$ is the amplitude of the resonance signal. This method is based on the electric resonance method, first used in molecular beams to observe electric dipole transitions between states of the same parity [15]. It has also been used to measure Rydberg fine structure intervals using radio frequency electric fields [9]. The essential idea is that the (on resonance) Rabi frequency $\Omega$ for the transition is proportional to the static field $E_{S}$; i. e. $\Omega \propto E_{S}$. In the presence of the static field $E_{S}$ a single microwave photon can be used to drive the $(n+1) d_{5 / 2} \rightarrow n g$ transition, and the Rabi frequency is given by

$$
\Omega=\frac{\left\langle(n+1) d\left|\mu E_{m w}\right| n f\right\rangle\left\langle n f\left|\mu E_{S}\right| n g\right\rangle}{W_{n g}-W_{n f}},
$$

from which it is evident that if $E_{m w}$ is fixed, $\Omega \propto E_{S}$. We ignore the small variations due to the relative orientations of $\vec{E}_{S}$ and $\vec{E}_{m w}$.

If the microwave field is present for a time $T$, and $\Omega T \ll \pi$, then the transition probability, and the magnitude of the $(n+1) d_{5 / 2} \rightarrow n g$ resonance signal $S$, is 
proportional to $(\Omega T)^{2}$, which is proportional to $E_{S}^{2}$, so in this case $X \propto E_{S}^{2}$. In sum,

$$
S \propto E_{S}^{2} .
$$

A plot of the resonance frequency $\nu_{d_{5 / 2} \rightarrow g}$ vs $S$ should be a straight line, the intercept of which is the zero field $(n+1) d_{5 / 2} \rightarrow n g$ interval. As we shall see, this method, which requires lower fields and thus smaller extrapolations, is the preferred approach.

\subsection{Experimental Approach}

In the experiment, ${ }^{85} \mathrm{Rb}$ atoms in a vapor-loaded magneto-optical trap (MOT) are held at the center of four vertical rods as shown in Fig. 5.1 [28]. The rods pass through the corners of a horizontal square $18 \mathrm{~mm}$ on a side. The two rods opposite the microchannel plate $(\mathrm{MCP})$ detector are connected together (inside the vacuum chamber) and are used primarily to apply a field ionization pulse, although a DC bias voltage can also be applied. The two rods closest to the MCP are also connected together and can be grounded or biased to provide a static field.

The direction of the applied field is horizontal and parallel to the axis of the MCP. For simplicity, throughout this chapter, we define the horizontal axis along the static electric field as the $z$ direction as shown in Fig. 5.1. With this rod configuration, which is functionally equivalent to a pair of plates, we can only null the stray field in the $z$ direction.

The $780 \mathrm{~nm}$ trap lasers are on continuously, and $\mathrm{Rb} 5 p_{3 / 2}$ atoms in the MOT are excited to the Rydberg $(n+1) d_{5 / 2}$ states by the a $10 \mu J 480 \mathrm{~nm}$ laser pulse 
at a $20-\mathrm{Hz}$ repetition rate. The laser pulse is $10 \mathrm{~ns}$ long with bandwidth of 150 MHz. The trap magnetic fields are switched off $\sim 4$ ms before the laser pulse is fired. The trap fields in the MOT have fallen to less than $50 \mathrm{mG}$ by the time the pulsed laser fires. After the laser is fired, atoms are exposed to the 500-ns microwave pulse to drive the $(n+1) d_{5 / 2} \rightarrow n g$ and $n h$ transitions, as shown in Fig. 5.2. The continuous microwaves are generated by an Agilent E824C PSG CW synthesized signal generator which produces frequencies up to $20 \mathrm{GHz}$. The microwaves are then formed into a 500-ns pulse by a General Microwave DM862B switch. The microwave frequency is doubled by a Narda 2640X220 active doubler and then tripled by Pacific Millimeter W3WO passive tripler to reach frequencies in the range of 75$110 \mathrm{GHz}$. The microwave pulse propagates through WR10 waveguide and is launched from outside the vacuum chamber into the MOT volume by the WR10 horn. The polarization of the microwave field is nominally in the $z$ direction, although, due to scattering from the rods the polarization is not well known when the microwaves reach the atoms in the MOT. During the experiment the applied static electric field is always present. We ionize the Rydberg atoms and detect ions by applying a 3- $\mu$ s rise time positive high voltage pulse to the rods $\sim 50$ ns after the end of the microwave pulse. The resulting ions are driven to the MCP detector. The signal from the MCP is recorded with a gated integrator and stored in a computer for analysis. 


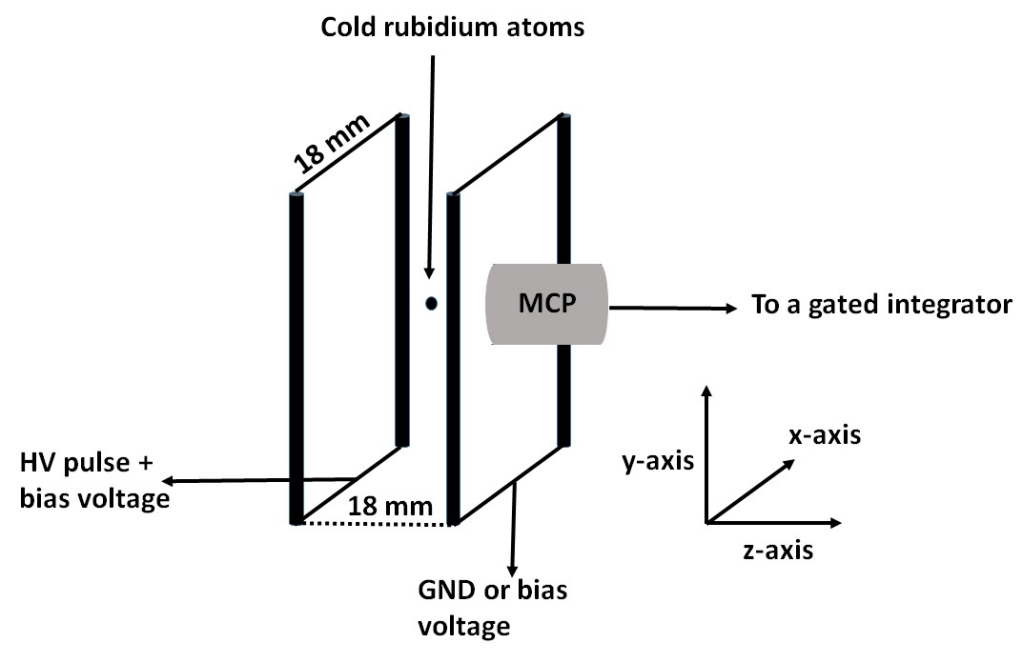

Figure 5.1: MOT configuration of this experiment.

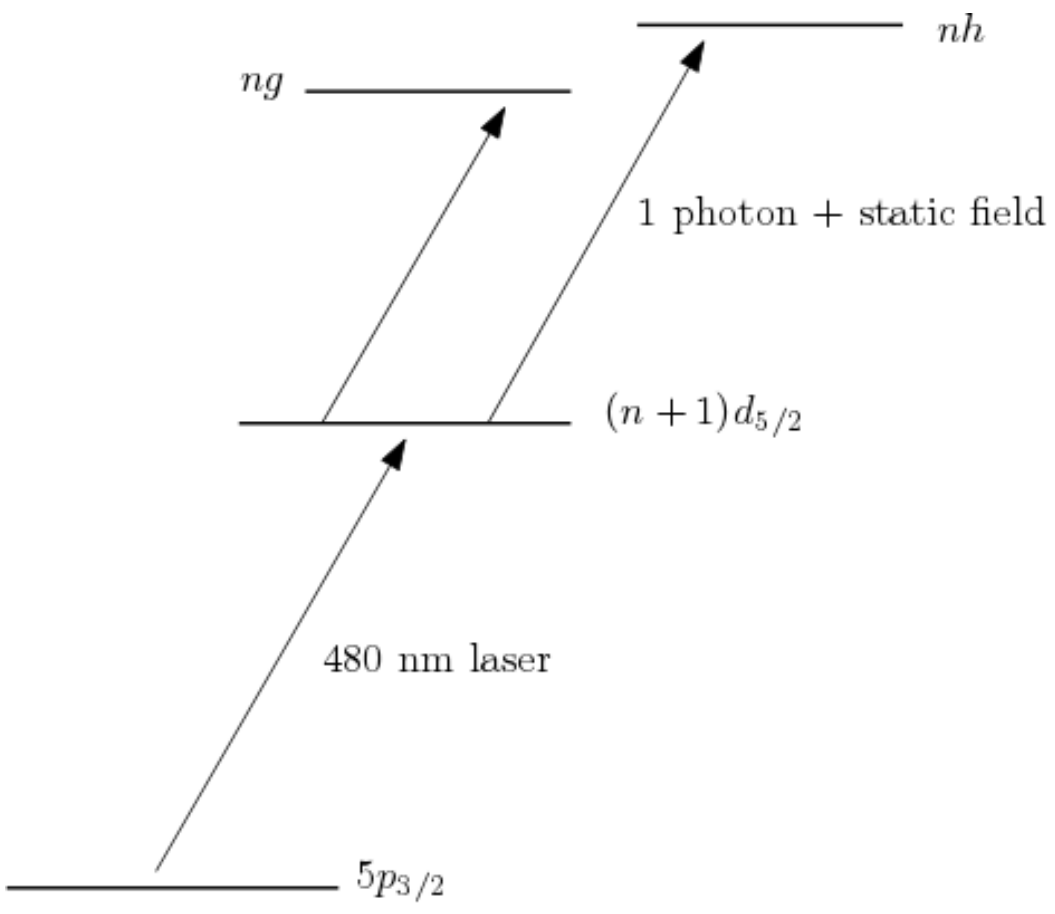

Figure 5.2: Schematic of the Rydberg energy levels of this experiment. 


\subsection{Experimental Observations and Discussion}

\subsubsection{Comparison of methods for determining the zero field $(n+1) d_{5 / 2}-n g$ intervals}

In this subsection we use the $29 d_{5 / 2} \rightarrow 28 g$ transition as a concrete example of different approaches to finding the zero field intervals. Since the MOT configuration allows us to control the static field in only one direction $\left(\vec{E}_{z}\right)$, we first observe the frequency of the $29 d_{5 / 2} \rightarrow 28 \mathrm{~g}$ transition as a function of bias voltage applied to the rods, changing the field in the $z$ direction from positive to negative. Although $d_{5 / 2} \rightarrow g$ transitions are more commonly driven as two microwave photon transitions [8], we drive them using one microwave photon and a static field, which we can vary by changing the bias voltage $V_{b}$. The observed frequencies of the resonances show the expected quadratic dependence on the bias voltage, as shown in Fig. 5.3. Fitting the observed frequencies to a quadratic dependence on the bias voltage gives 104 $371.08(40) \mathrm{MHz}$ as the maximum frequency at a bias voltage of $V_{b}=V_{0}=0.24 \mathrm{~V}$ and $104367.74(40) \mathrm{MHz}$ as the frequency when there is no bias voltage $\left(V_{b}=0\right)$. At $V_{b}=V_{0}=0.24 \mathrm{~V}$ the stray field in the $z$ direction is nulled. For our rod geometry the conversion between bias voltage and bias field, the correction to $E_{z}$, at the MOT is $E_{b}(\mathrm{~V} / \mathrm{cm})=0.406 V_{b}(\mathrm{~V})$. Accordingly, the original stray field in the $z$ direction is $97 \mathrm{mV} / \mathrm{cm}$, which leads to frequency shift of $3.34 \mathrm{MHz}$. The frequency 104371.08 $\mathrm{MHz}$ is only a lower limit to the $29 d_{5 / 2}-28 g$ frequency since the stray field in the $x-y$ plane, $E_{\perp}$, is unknown. One might reasonably assume that the original stray field had approximately equal components in all three directions, in which case the 


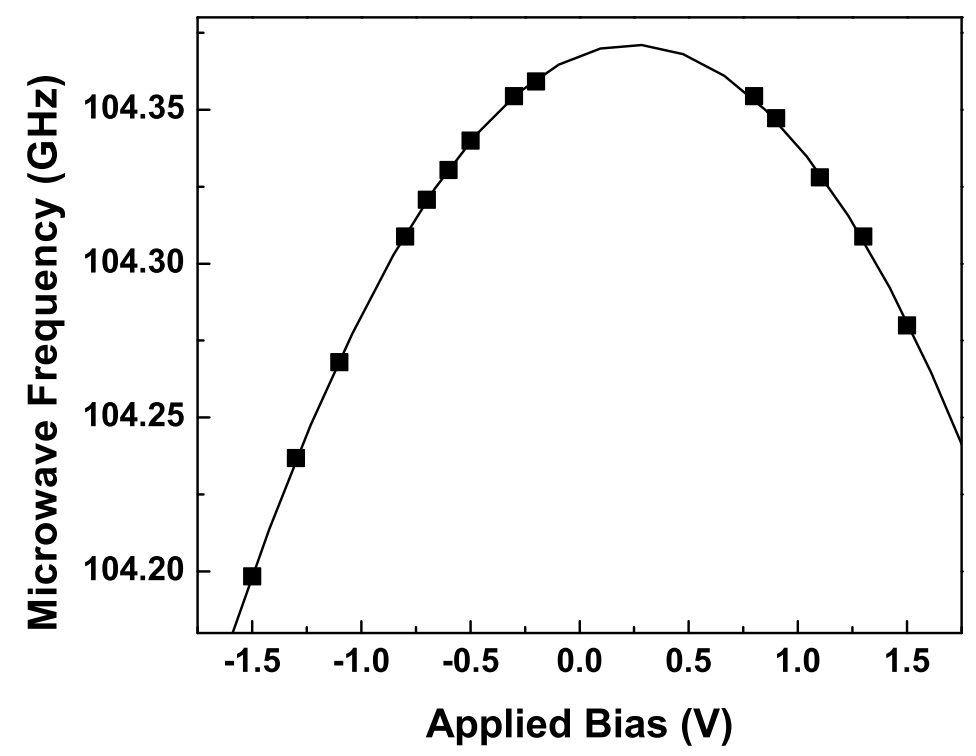

Figure 5.3: The frequency of the microwave $29 d_{5 / 2} \rightarrow 28 g$ transition vs bias electric field in the $z$ direction. The maximum frequency is $104371.08(40) \mathrm{MHz}$ at a bias voltage of $V_{b}=V_{0}=0.24 \mathrm{~V}$. At this bias, $E_{z}$ is nulled.

uncompensated stray field would be $137 \mathrm{mV} / \mathrm{cm}$, leading to an additional frequency shift of $6.68 \mathrm{MHz}$. Correcting for this assumed uncompensated shift gives a zero field $29 d_{5 / 2}-28 g$ interval of $104377.76 \mathrm{MHz}$. Assigning an uncertainty presents a problem, but an uncertainty equal to correction for $E_{\perp}, 6.7 \mathrm{MHz}$ does not seem unreasonable, yielding 104377.8(67) $\mathrm{MHz}$ as the final result for the zero field interval.

To take into account the fields in all directions we use the approaches described in Section II. In the first approach we implicitly determine $E_{S}$ from $\Delta \nu_{S}$, the separation of adjacent Stark states. For each bias voltage we observe a Stark spectrum, as shown in Fig. 5.4(a), and the $d_{5 / 2} \rightarrow g$ transition with a lower microwave power in Fig. 
5.4(b). The $d_{5 / 2} \rightarrow g$ transition also appears in Fig. 5.4(a), but it is power broadened when the high $k$ states are visible. The frequency separation $\Delta \nu_{S}$ between adjacent Stark states is proportional to $E_{S}$. For each bias voltage we obtain values of the $d_{5 / 2} \rightarrow g$ frequency $\nu_{d_{5 / 2} \rightarrow g}$ and $\Delta \nu_{S}$, taken from the high $k$ states indicated in Fig. 5.4(a). From these pairs of points we construct the parametric plot of the $d_{5 / 2} \rightarrow g$ frequency vs $\Delta \nu_{S}^{2}$. As shown in Fig. 5.5, the result is a straight line, as expected from Eq. (5.2), and its intercept is the zero field $d_{5 / 2} \rightarrow g$ interval. From Fig. 5.5, we obtain the zero field $29 d_{5 / 2} \rightarrow 28 \mathrm{~g}$ transition frequency to be $104378.9(62) \mathrm{MHz}$.

This approach has the attraction that we are measuring frequencies, but it has the obvious problem that the fields must be large enough to obtain good values for the separation between the Stark states. The relatively large fields require a long extrapolation, $40 \mathrm{MHz}$, to zero field, and they introduce the possibility that the Stark shift of the transition frequency may not be adequately represented by Eq. (5.2). A variant of this technique is to conduct the Stark spectroscopy at higher $n$, where the separations are larger, allowing the use of smaller fields. This approach has been used by Stevens and Lundeen to monitor static fields [34].

To conduct measurements in lower static fields we use the second approach described in Section II. Specifically, we measure the signal amplitude $S$ of the $(n+1) d_{5 / 2} \rightarrow n g$ transition at different bias voltages. Since we are driving the transition with one microwave photon and a static field, for a fixed microwave amplitude the Rabi frequency is proportional to the static field, as shown by Eq. (5.4). The experiment is conducted in the low transition probability regime in which $S$ is proportional to $E_{S}^{2}$. The procedure is similar to that used for the Stark spectroscopy 


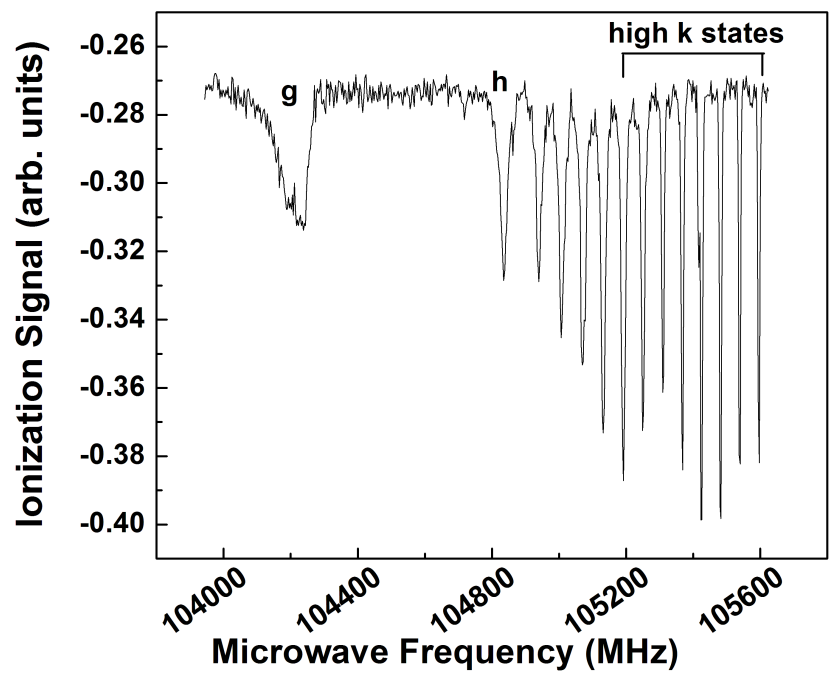

(a)

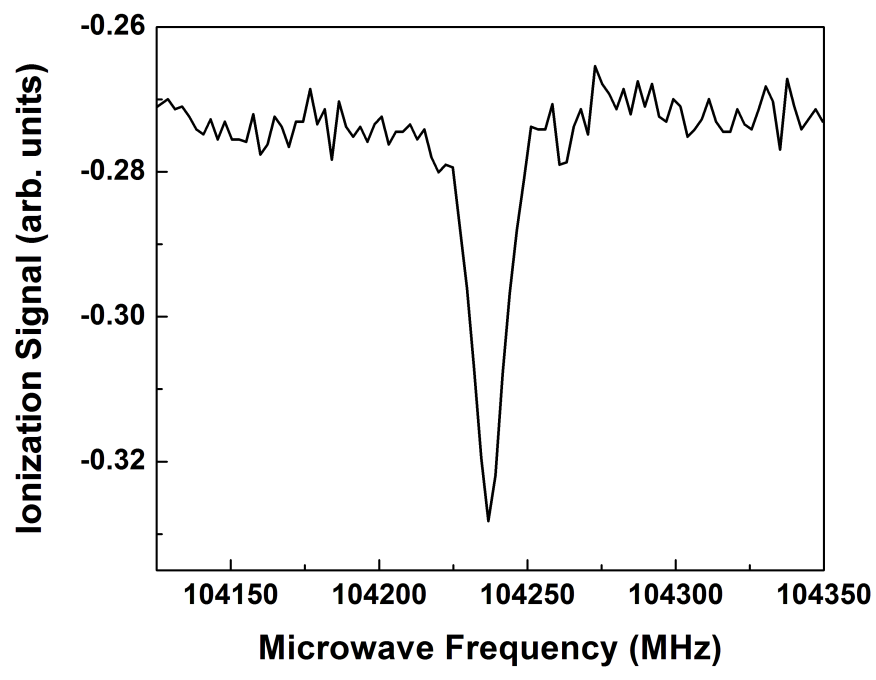

(b)

Figure 5.4: (a) The $29 d_{5 / 2} \rightarrow 28 k$ Stark spectrum at bias voltage of $V_{b}=-1.3 \mathrm{~V}$ at the relative microwave power 1 . The high $k$ states are indicated in the graph. (b) The $29 d_{5 / 2} \rightarrow 28 g$ transition at the same bias field as (a) but a relative microwave power of 0.032 . 


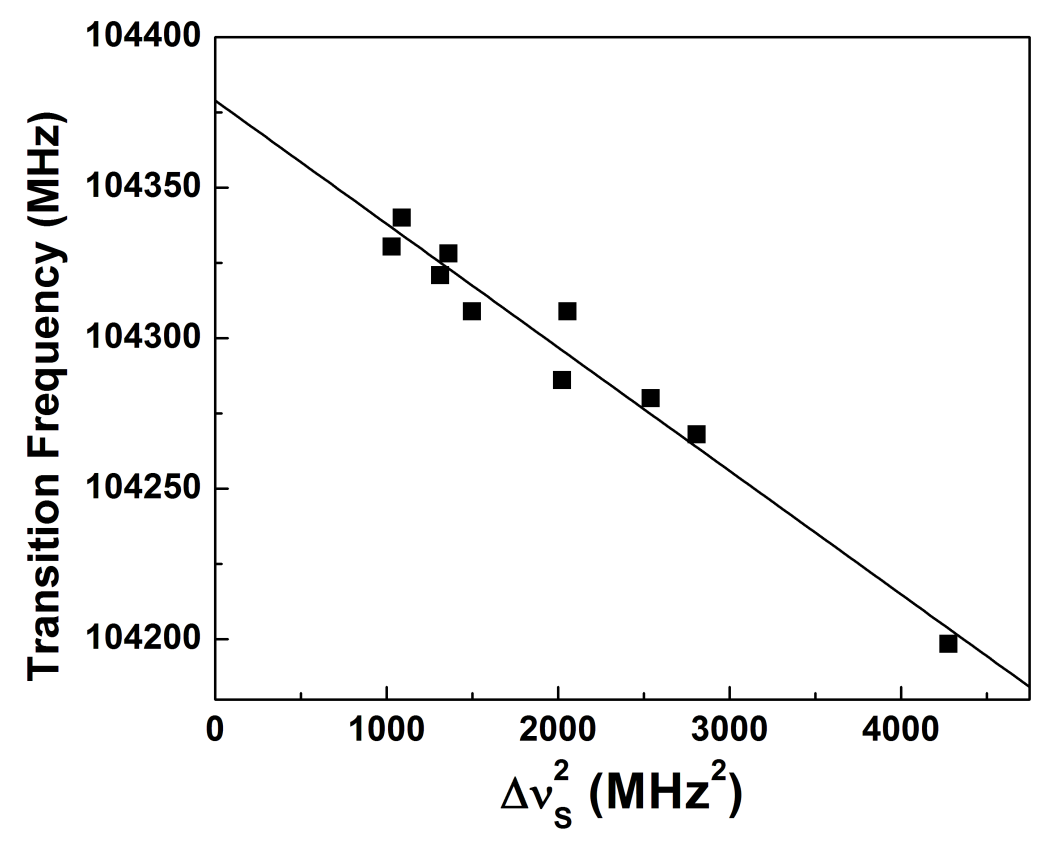

Figure 5.5: The $29 d_{5 / 2} \rightarrow 28 \mathrm{~g}$ transition frequency vs the square of the Stark splitting of the high $k$ states $\left(\Delta \nu_{S}^{2}\right)$ obtained from Stark spectroscopy. At $\Delta \nu_{S}^{2}=0$, the stray field is zero and the $29 d_{5 / 2} \rightarrow 28 \mathrm{~g}$ transition frequency is 104 378.9(62) MHz. 
approach. For each bias voltage we observe the $d_{5 / 2} \rightarrow g$ resonance, as shown in Fig. 5.6(a) for bias voltages between 0.34 and 0.61 V. As shown by Fig. 5.6(a), the signal amplitude $S$ increases and the resonance frequency $\nu_{d_{5 / 2} \rightarrow g}$ shifts with increasing bias voltage. Since $S \propto E_{S}^{2}$, a parametric plot of $\nu_{d_{5 / 2} \rightarrow g}$ vs $S$ yields a straight line, the intercept of which is the zero field $d_{5 / 2} \rightarrow g$ frequency as shown in Fig. 5.6(b). From Fig. 5.6(b), the zero field $29 d_{5 / 2} \rightarrow 28 g$ frequency is $104372.70(28) \mathrm{MHz}$. The extrapolation in this method is $\sim 4 \mathrm{MHz}$ which is much less than the aforementioned approach, which results in a smaller uncertainty in determining the the zero field $29 d_{5 / 2} \rightarrow 28 g$ frequency.

In Fig. 5.6 we have not explicitly used the bias voltages, but from them we can extract the values of the perpendicular stray field. When the observed frequencies are plotted vs the bias voltages $V_{b}$ we obtain a parabola similar to Fig. 5.3, with the maximum frequency of $104369.87(44) \mathrm{MHz}$ occurring at $V_{b}=V_{0}=0.875 \mathrm{~V}$. Since $S$ is proportional to $E_{S}^{2}$, we write

$$
S=a E_{S}^{2}=a\left(E_{z}^{2}+E_{\perp}^{2}\right),
$$

where $a$ is a constant. In Fig. 5.7 we plot $S$ vs $\left(V_{b}-V_{0}\right)^{2}$, which is in effect a plot of $S$ vs $E_{z}^{2}$, as shown by the horizontal scale at the top of the figure. At the $S$ intercept of the graph $E_{z}=0$ and $E_{S}^{2}=E_{\perp}^{2}$. The slope $a=d S / d E_{S}^{2}$, combining these two values from Fig. 5.7, we obtain $E_{\perp}=91 \mathrm{mV} / \mathrm{cm}$. We can check this value for $E_{\perp}$ using a different approach. With $V_{b}=0$ the observed frequency is $104326.05 \mathrm{MHz}$. With $V_{b}=V_{0}=0.875 \mathrm{~V}$, so that $E_{z}=0 \mathrm{mV} / \mathrm{cm}$, results in a shift of $43.82 \mathrm{MHz}$. To reach the zero field value requires a further shift of $2.83 \mathrm{MHz}$, which implies that $E_{\perp}=90$ 


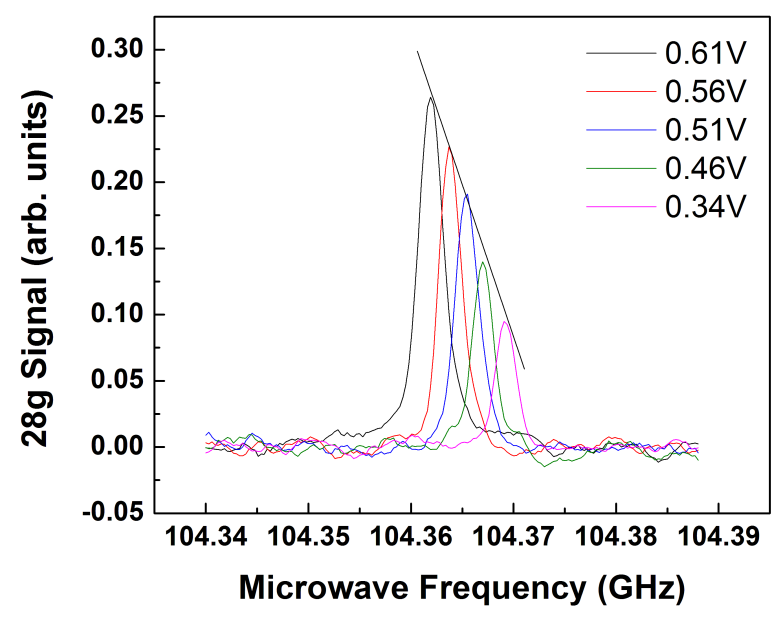

(a)

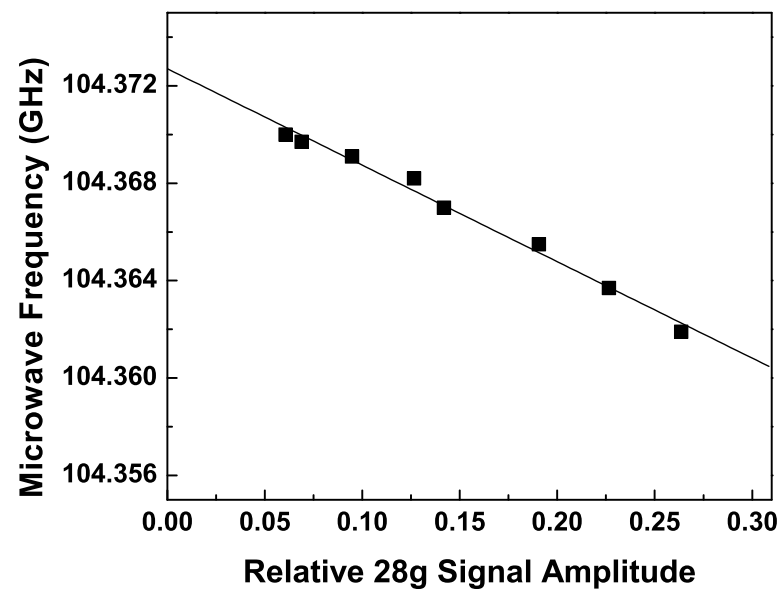

(b)

Figure 5.6: (a) The observed the $29 d_{5 / 2} \rightarrow 28 \mathrm{~g}$ resonance signals with different bias voltages. As shown by the slanted line, the signal amplitude varies linearly with the observed resonance frequency. The resonance frequency increases and the signal amplitude decreases as the bias voltage is reduced from $V_{b}=0.61 \mathrm{~V}$ to $0.34 \mathrm{~V}$, which reduces $E_{z}$ from $150 \mathrm{mV} / \mathrm{cm}$ to $41 \mathrm{mV} / \mathrm{cm}$. (b) The $29 d_{5 / 2} \rightarrow 28 \mathrm{~g}$ microwave transition frequency as a function of the relative $28 \mathrm{~g}$ signal amplitude. From the graph, the $29 d_{5 / 2} \rightarrow 28 \mathrm{~g}$ transition frequency at zero stray field is $104372.70(28)$ $\mathrm{MHz}$. 
$\mathrm{mV} / \mathrm{cm}$, in good agreement with the value given above. It is instructive to apply the same method of analysis to the data of Fig. 5.3, which leads to $E_{\perp}=68 \mathrm{mV} / \mathrm{cm}$. The data shown in Fig. 5.3 and Fig. 5.6 were taken with opposite polarity field ionization pulses, which result in different bias fields in the $z$ direction. Nonetheless the values for $E_{\perp}$ are similar. In addition the values for $E_{\perp}$ are smaller than the bias field in the $z$ direction due to the fact that the bias field in the $z$ direction is determined by an external circuit.

At this point it is useful to compare the three approaches we have described. The first method, measuring transition frequency as a function of bias voltage, allows the determination of the $29 d_{5 / 2} \rightarrow 28 \mathrm{~g}$ frequency with high precision, but the observed frequency is the zero field $29 d_{5 / 2}-28 g$ interval altered by an unknown Stark shift due to the uncanceled stray field $E_{\perp}$. Estimating the Stark shift due to $E_{\perp}$ by assuming that the magnitudes of the stray field $\left|E_{x}\right|,\left|E_{y}\right|$ and $\left|E_{z}\right|$ are the same, we arrived at a zero field $29 d_{5 / 2}-28 g$ interval of $104377.8(67) \mathrm{MHz}$. The second method, measuring transition frequency as a function of separation of adjacent Stark states, yields a $29 d_{5 / 2} \rightarrow 28 \mathrm{~g}$ frequency transition of $104378.9(62) \mathrm{MHz}$. This method has the distinct advantage of actually measuring $\left|E_{\perp}\right|$, but it has the disadvantage of requiring a long extrapolation to zero field, which results in an uncertainty of 6.2 $\mathrm{MHz}$, not really much better than the estimated uncertainty of the first method. The third method, measuring transition frequency as a function of the signal amplitude, yields a $29 d_{5 / 2} \rightarrow 28 \mathrm{~g}$ frequency transition of $104372.70(28) \mathrm{MHz}$. The shorter extrapolation results in a smaller uncertainty, $0.28 \mathrm{MHz}$, of the the zero field $29 d_{5 / 2} \rightarrow$ $28 g$ frequency and using this method we estimate $E_{\perp}$ to be $91 \mathrm{mV} / \mathrm{cm}$, slightly 


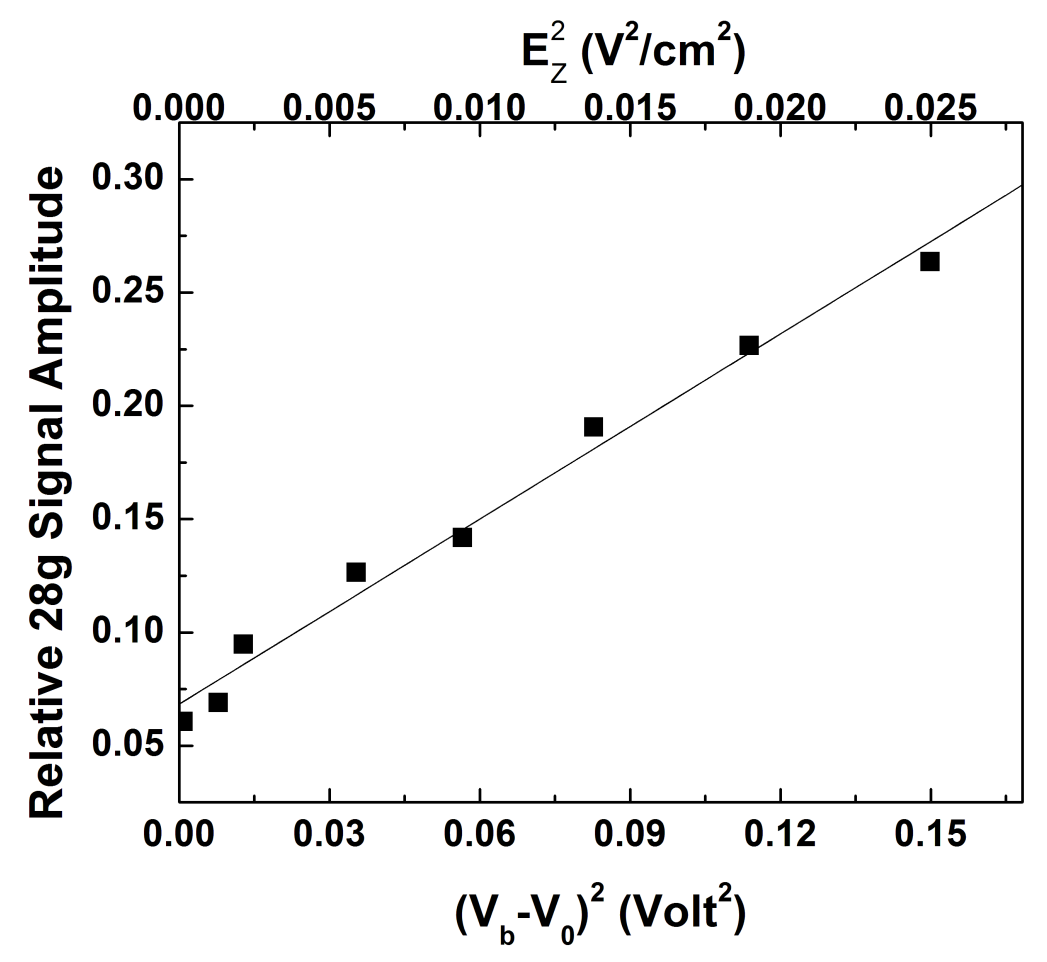

Figure 5.7: The graph of the relative $28 \mathrm{~g}$ signal amplitude $(S)$ as a function of squared voltage $\left(V_{b}-V_{0}\right)^{2}$ and squared static field $E_{z}^{2}$ in the $z$ direction of the system. Since $S=a\left(E_{z}^{2}+E_{\perp}^{2}\right)$, from the intercept and slope of the graph we determine $E_{\perp}$ to be $91 \mathrm{mV} / \mathrm{cm}$. 
less than our estimate of $137 \mathrm{mV} / \mathrm{cm}$ made on the basis of simply measuring the frequency vs the bias voltage $V_{b}$. Comparing the three methods shows that the third method, unlike the first method, has a known uncertainty and it is a factor of twenty smaller than the uncertainty of the second method. Consequently, we use the third method to determine the zero field intervals in the sections that follow. Finally, we note that, although the stray field varies from day to day, the zero field intervals extracted remain constant, within their uncertainties.

\subsubsection{The $(n+1) d_{5 / 2}-n g$ intervals and $n g$ quantum defects}

While measuring signal strengths is less appealing than measuring frequencies, as in the separation between the Stark states, the much lower static fields make the signal amplitude method more attractive, and we have used it to measure the $(n+1) d_{5 / 2} \rightarrow$ $n g$ transition frequencies for $27 \leq n \leq 30$, as shown in Table 5.1. To find the quantum defects of the $n g$ states, we add the known quantum defects of the $n d$ states from Ref. [20] and the $(n+1) d_{5 / 2} \rightarrow n g$ transition frequencies. The values of the quantum defects of the $n g$ states of $27 \leq n \leq 30$ are shown in Table 5.1. Ref $[1,13,17]$ measured quantum defects of the $n g$ states to be $0.00400(9), 0.00405(6)$ and 0.00402(8), respectively. Ref $[13,17]$ did not consider $n$ dependence and Ref. [1] measured only the $30 \mathrm{~g}$ quantum defect. As seen from Table 5.1, our values of the quantum defect have improved upon the previous measurements by an order of magnitude.

Our quantum defects show a slight $n$ dependence, and we fit them to a Ritz 
Table 5.1: The $(n+1) d_{5 / 2} \rightarrow n g$ microwave transition frequencies in zero stray electric field and the extracted quantum defects of $n g$.

\begin{tabular}{crc}
\hline \hline$n$ & Transition frequency $(\mathrm{MHz})$ & Quantum defect \\
\hline 27 & $116464.54(35)$ & $0.0039737(11)$ \\
28 & $104372.70(28)$ & $0.0039701(10)$ \\
29 & $93895.75(34)$ & $0.0039746(13)$ \\
30 & $84775.26(65)$ & $0.0039778(27)$ \\
\hline \hline
\end{tabular}

formula,

$$
\delta_{g}=\delta_{0}+\frac{\delta_{1}}{\left(n-\delta_{0}\right)^{2}},
$$

which yields the values $\delta_{0}=0.00400(2)$ and $\delta_{1}=-0.018(15)$.

\subsubsection{The $(n+1) d_{5 / 2}-n h$ intervals and $n h$ quantum defects}

We obtain the zero field $(n+1) d_{5 / 2} \rightarrow n h$ intervals in much the same way as we obtained the $(n+1) d_{5 / 2} \rightarrow n g$ intervals. We drive the $d_{5 / 2} \rightarrow h$ transitions using one microwave photon and a static field. The Rabi frequency $\Omega$ is given by

$$
\Omega=\frac{\left\langle d\left|\mu E_{m w}\right| f\right\rangle\left\langle f\left|\mu E_{s}\right| g\right\rangle\left\langle g\left|\mu E_{s}\right| h\right\rangle}{\left(W_{n g}-W_{n f}\right)\left(W_{n h}-W_{n g}\right)} .
$$

In this case $\Omega \propto E_{S}^{2}$, and in the small transition probability regime $S \propto E_{S}^{4}$. The experiment is conducted in much the same way as the $d_{5 / 2} \rightarrow g$ measurements; for different bias voltages the signal amplitude $S$ and resonance frequency $\nu_{d_{5 / 2} \rightarrow h}$ are measured while keeping the microwave field amplitude fixed. Fig. 5.8 shows the $29 d_{5 / 2} \rightarrow 28 h$ transition as an example. A parametric plot of $\nu_{d_{5 / 2} \rightarrow h}$ vs $\sqrt{S}$ should give a straight line, the intercept of which is the the zero field $d_{5 / 2}-h$ interval, and 


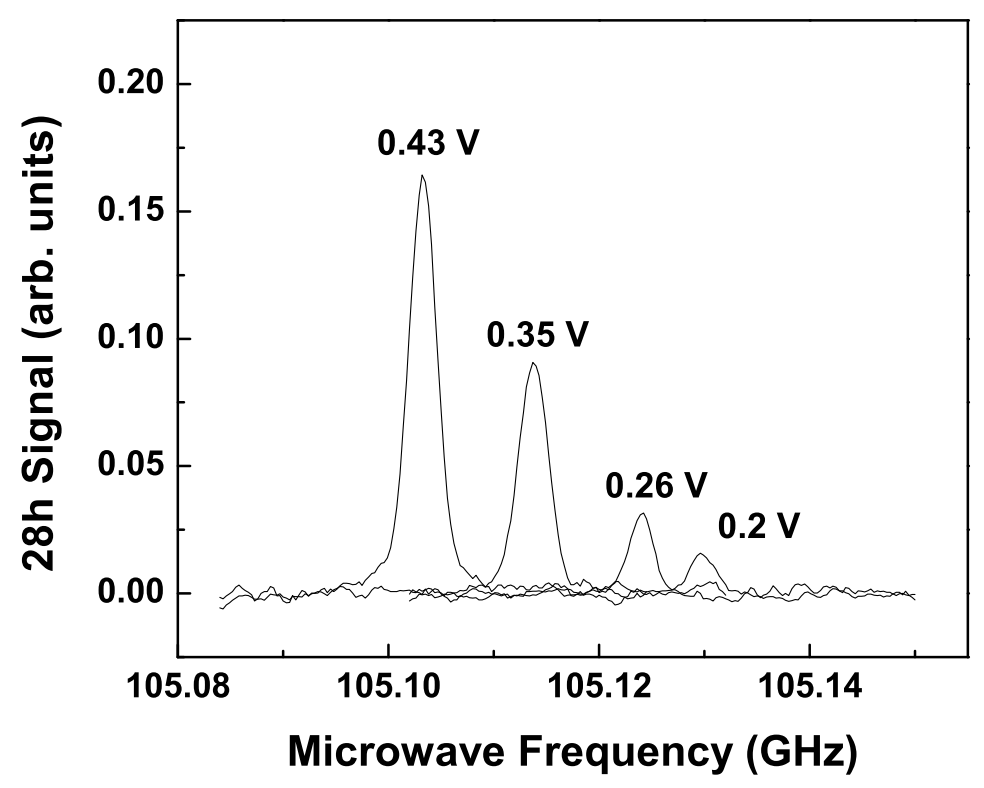

Figure 5.8: The observed amplitude as the $29 d_{5 / 2} \rightarrow 28 h$ resonance changes in different bias voltages.

in Fig. 5.9 we present this plot for the $29 d_{5 / 2} \rightarrow 28 h$ transition. As shown, the plot matches our expectation and yields the zero field interval of 105 140.87(77) MHz. Following the same procedure we have measured the $(n+1) d_{5 / 2} \rightarrow n h$ intervals for $28 \leq n \leq 30$, with the results shown in Table 5.2. In Table 5.2 we also give the $n h$ quantum defects, obtained in a manner analogous to that used to obtain the $n g$ quantum defects. 


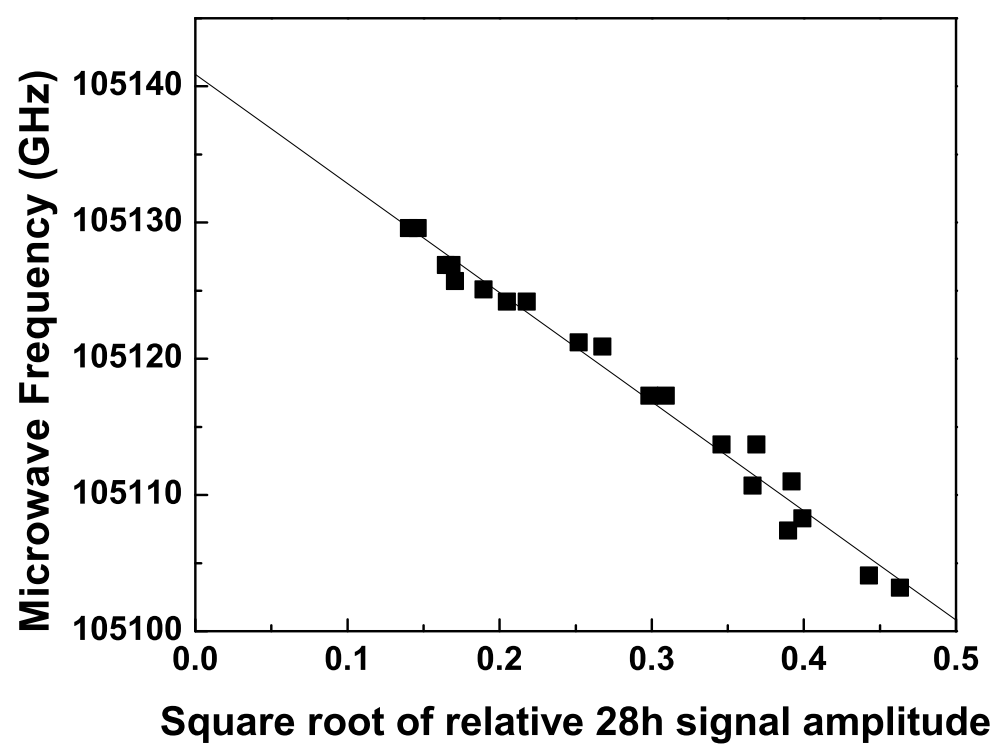

Figure 5.9: The frequency of the observed $29 d_{5 / 2} \rightarrow 28 h$ resonances as a function of the square root of the relative $28 h$ signal amplitude, $\sqrt{S}$. From the graph, the $29 d_{5 / 2} \rightarrow 28 h$ transition frequency at zero stray field is $105140.87(77) \mathrm{MHz}$.

Table 5.2: The $(n+1) d_{5 / 2} \rightarrow n h$ microwave transition frequencies in zero stray electric field and the extracted quantum defects of $n h$.

\begin{tabular}{ccc}
\hline \hline$n$ & Transition frequency $(\mathrm{MHz})$ & Quantum defect \\
\hline 28 & $105140.9(8)$ & $0.0014078(27)$ \\
29 & $94591.0(22)$ & $0.0013982(82)$ \\
30 & $85400.3(15)$ & $0.0014137(62)$ \\
\hline \hline
\end{tabular}




\subsubsection{The $\mathrm{Rb}^{+}$ionic dipole and quadrupole polarizabilities}

We use the values of quantum defects that we have determined and the adiabatic core polarization model of Mayer and Mayer to extract the ionic dipole and quadrupole polarizabilities of $\mathrm{Rb}^{+}[22]$. For the high $\ell$ states, where $\ell>3$, the energy levels of the $\mathrm{Rb}$ atoms are depressed from the hydrogenic levels only by core polarization; core penetration is negligible. In Rb the Rydberg electron moves much more slowly than the electrons in the core, and the $\mathrm{Rb}^{+}$core is polarized by the slowly varying field from the Rydberg electron. The polarization interaction between the Rydberg electron and the ion core depresses the energy of the Rb $n \ell$ Rydberg state below the energy of a hydrogenic $n \ell$ state by

$$
W_{\mathrm{pol}, n \ell}=-\frac{\alpha_{d}}{2}\left\langle 1 / r_{n \ell}^{4}\right\rangle-\frac{\alpha_{q}}{2}\left\langle 1 / r_{n \ell}^{6}\right\rangle
$$

where $\alpha_{d}$ and $\alpha_{q}$ are the ionic dipole and quadrupole polarizabilities. The expectation values of $\left\langle 1 / r_{n \ell}^{4}\right\rangle$ and $\left\langle 1 / r_{n \ell}^{6}\right\rangle$ are the squares of the field and the field gradient of the Rydberg electron in the $n \ell$ state at the core. The resulting energy of the Rb $n \ell$ state is given by

$$
W_{n \ell}=-1 / 2 n^{2}+W_{\mathrm{pol}, n \ell} .
$$

The energy levels of the Rydberg $n \ell$ state can also be expressed as

$$
W_{n \ell}=-1 / 2\left(n-\delta_{n \ell}\right)^{2},
$$


where $\delta_{n \ell}$ is the quantum defect of the Rydberg $n \ell$ state. Since $n$ is much larger than $\delta_{n \ell}$, using a Taylor expansion we can express the polarization energy as

$$
W_{\mathrm{pol}, n \ell}=1 / 2 n^{2}+W_{n \ell} \cong-\frac{\delta_{n \ell}}{n^{3}}
$$

From Eqs. (5.9) and (5.12), we get

$$
\frac{\delta_{n \ell}}{n^{3}}=\frac{\alpha_{d}}{2}\left\langle 1 / r_{n \ell}^{4}\right\rangle+\frac{\alpha_{q}}{2}\left\langle 1 / r_{n \ell}^{6}\right\rangle .
$$

We can rewrite Eq. (5.13) as

$$
2 \frac{\delta_{n \ell}}{n^{3}\left\langle 1 / r_{n \ell}^{4}\right\rangle}=\alpha_{d}+\alpha_{q} \frac{\left\langle 1 / r_{n \ell}^{6}\right\rangle}{\left\langle 1 / r_{n \ell}^{4}\right\rangle}
$$

Eq. (5.14) implies that a graph of $2 \delta_{n \ell} /\left(n^{3}\left\langle r_{n \ell}^{-4}\right\rangle\right)$ vs $\left\langle r_{n \ell}^{-6}\right\rangle /\left\langle r_{n \ell}^{-4}\right\rangle$ is linear, with $\alpha_{d}$ as the intercept and $\alpha_{q}$ as the slope of the graph. Here the values of $\delta_{n \ell}$ are the experimentally determined values given above for $27 \leq n \leq 30, l=4$ and 5 . We use the known analytic expressions for $\left\langle r_{n \ell}^{-4}\right\rangle$ and $\left\langle r_{n \ell}^{-6}\right\rangle$ for the $n \ell$ states of hydrogen $[3,7]$. In Fig. 5.10 we plot the graph of $2 \delta_{n \ell} /\left(n^{3}\left\langle r_{n \ell}^{-4}\right\rangle\right)$ vs $\left\langle r_{n \ell}^{-6}\right\rangle /\left\langle r_{n \ell}^{-4}\right\rangle$, and we determine the ionic dipole and quadrupole polarizabilities to be $\alpha_{d}=9.12(2) a_{0}^{3}$ and $\alpha_{q}=14(3) a_{0}^{5}$, respectively. In Table 5.3, we compare the $\mathrm{Rb}^{+}$ionic dipole and quadrupole polarizabilities obtained from our work to other theoretical and experimental work. Our ionic dipole polarizability agrees with the earlier experimental determinations but has a much smaller uncertainty, and it agrees very well with the theoretical predictions. Refs. $[24,31]$ contain excellent summaries of the theory of 


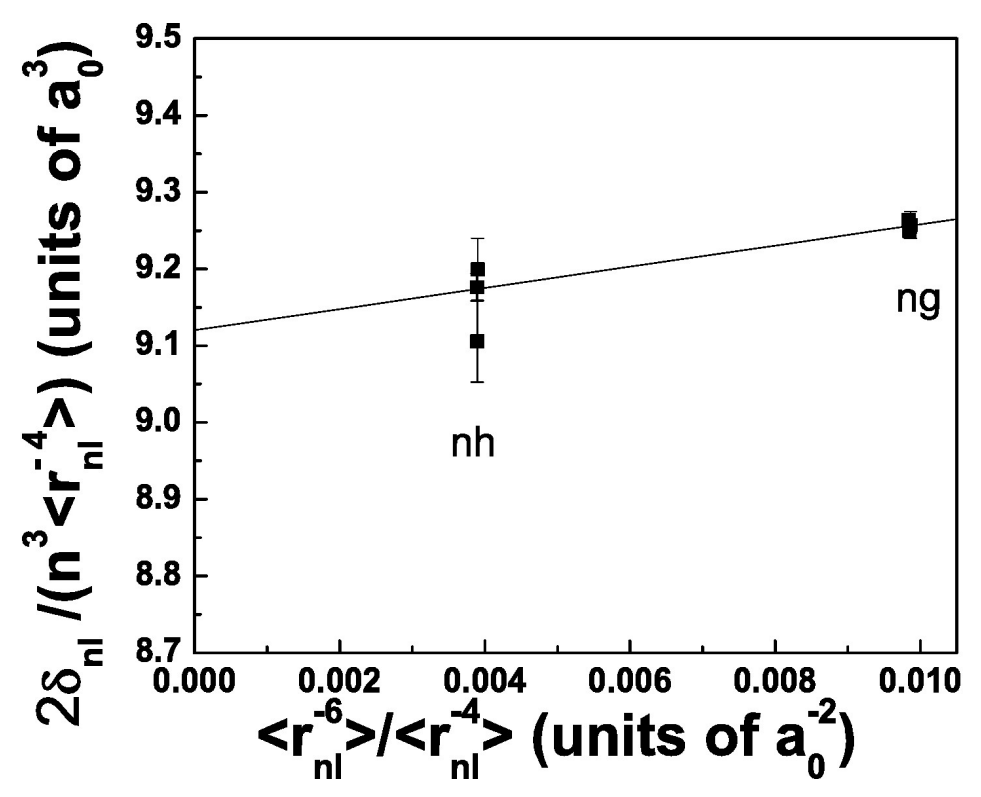

Figure 5.10: A plot of the measured $n g$ and $n h$ quantum defects scaled by $n^{3}\left\langle r_{n \ell}^{-4}\right\rangle / 2$ vs $\left\langle r_{n \ell}^{-6}\right\rangle /\left\langle r_{n \ell}^{-4}\right\rangle$ using Eq. (5.14). There are 3 data points for the $n h$ quantum defects, $28 \leq n \leq 30$, and 4 data points for the $n g$ quantum defects, $27 \leq n \leq 30$. A fit to the straight line yields the y-intercept and slope, which are $\alpha_{d}$ and $\alpha_{q}$, respectively. The resulting fit values are $\alpha_{d}=9.12(2) a_{0}^{3}$ and $\alpha_{q}=14(3) a_{0}^{5}$.

ionic polarizabilities. The experimental values for the ionic dipole polarizability from Refs. $[13,16,17]$ are determined from the $n f$ and $n g$ energy levels using the core polarization model. Although the $n f$ states are core penetrating states which should not be treated using the core polarization analysis alone, the values obtained for $\alpha_{d}$ are consistent with ours.

Our ionic quadrupole polarizability falls within the broad limits set in Refs. $[13,17]$ but is about a factor of two lower than the theoretical prediction. In the lat- 
Table 5.3: The $\mathrm{Rb}^{+}$dipole $\left(\alpha_{d}\right)$ and quadrupole $\left(\alpha_{q}\right)$ polarizabilities obtained from this work and other theoretical (Th) and experimental (Exp) results.

\begin{tabular}{lll}
\hline \hline & $\alpha_{d}\left(a_{0}^{3}\right)$ & $\alpha_{q}\left(a_{0}^{5}\right)$ \\
\hline This work & $9.12(2)$ & $14(3)$ \\
\hline Other works & $8.9<\alpha_{d}<9.3(\operatorname{Exp})[13]$ & $0<\alpha_{q}<43(\operatorname{Exp})[13]$ \\
& $8.5<\alpha_{d}<9.7(\operatorname{Exp})[17]$ & $0<\alpha_{q}<55(\operatorname{Exp})[17]$ \\
& $8.98(\operatorname{Exp})[16]$ & $35.4(\mathrm{Th})[31]$ \\
& $9.1(\mathrm{Th})[31]$ & $38.37(\mathrm{Th})[32]$ \\
& $9.11(\mathrm{Th})[21]$ & $35.41(\mathrm{Th})[18]$ \\
& $9.076(\mathrm{Th})[18]$ & \\
\hline
\end{tabular}

ter connection it is noteworthy that core polarization analyses of Rydberg quantum defects of other elements have consistently yielded ionic quadrupole polarizabilities that are lower than theoretically predicted $[5,19,26,27,30]$. It is a worthy theoretical challenge to pinpoint the source of the discrepancy between the theoretical and experimental values. We believe that the core polarization model needs to be reexamined closely. We hope this will motivate theorists to take a closer look at the core polarization model.

\subsection{Conclusion}

We have presented an experimental technique to determine zero field transition frequencies in spite of our inability to completely cancel the stray field. We use the technique to measure the microwave transition frequencies from the Rydberg $(n+1) d_{5 / 2}$ states to the Rydberg $n g$ and $n h$ states, $27 \leq n \leq 30$. The $n g$ and $n h$ quantum defects of the measured $n$ states are determined from the observed microwave transition frequencies. We extract the $\mathrm{Rb}^{+}$ionic dipole and quadrupole polarizabilities 
from the values of quantum defects to be $\alpha_{d}=9.12(2) a_{0}^{3}$ and $\alpha_{q}=14(3) a_{0}^{5}$, respectively. The $\mathrm{Rb}^{+}$dipole polarizability agrees well with recent theoretical values. However, the $\mathrm{Rb}^{+} \alpha_{q}$ is about a factor of 2 lower than the theoretical prediction. The discrepancy between theoretical and experimental values is consistent with the determined $\alpha_{q}$ of other elements using the core polarization analysis. We hope this work will motivate theoretical work to locate the source of discrepancy between the experimental and theoretical values of $\alpha_{q}$ in the core polarization analysis. 


\section{REFERENCES}

[1] Afrousheh, K., Bohlouli-Zanjani, P., Petrus, J. A., \& Martin, J. D. D. (2006). Determination of the ${ }^{85} \mathrm{Rb} n g$-series quantum defect by electric-field-induced resonant energy transfer between cold rydberg atoms. Phys. Rev. A, 74, 062712.

[2] Bethe, H. A. \& Salpeter, E. A. (1977). Quantum Mechanics of One- and Twoelectron Atoms. New York: Plenum Publishing Corporation.

[3] Bockasten, K. (1974). Mean values of powers of the radius for hydrogenic electron orbits. Phys. Rev. A, 9, 1087-1089.

[4] Bohlouli-Zanjani, P., Petrus, J. A., \& Martin, J. D. D. (2007). Enhancement of rydberg atom interactions using ac stark shifts. Phys. Rev. Lett., 98, 203005.

[5] Cooke, W. E., Gallagher, T. F., Hill, R. M., \& Edelstein, S. A. (1977). Resonance measurements of $d-f$ and $d-g$ intervals in lithium rydberg states. Phys. Rev. $A, 16,1141-1145$.

[6] Ekstrom, C. R., Schmiedmayer, J., Chapman, M. S., Hammond, T. D., \& Pritchard, D. E. (1995). Measurement of the electric polarizability of sodium with an atom interferometer. Phys. Rev. A, 51, 3883-3888.

[7] Gallagher, T. F. (1994). Rydberg Atoms. Cambridge, England: Cambridge University Press.

[8] Gallagher, T. F., Hill, R. M., \& Edelstein, S. A. (1976). Resonance measurements of $d-f-g-h$ splittings in highly excited states of sodium. Phys. Rev. A, 14, $744-750$.

[9] Gallagher, T. F., Humphrey, L. M., Hill, R. M., Cooke, W. E., \& Edelstein, S. A. (1977). Fine-structure intervals and polarizabilities of highly excited $p$ and $d$ states of sodium. Phys. Rev. A, 15, 1937-1944.

[10] Gould, H. \& Miller, T. M. (2005). Recent developments in the measurement of static electric dipole polarizabilities. volume 51 of Advances In Atomic, Molecular, and Optical Physics (pp. 343 - 361). Academic Press.

[11] Gray, L. G., Sun, X., \& MacAdam, K. B. (1988). Resonance measurements of d-f-g-h intervals in rydberg states of sodium and a redetermination of the core polarizabilities. Phys. Rev. A, 38, 4985-4993. 
[12] Gregoire, M. D., Hromada, I., Holmgren, W. F., Trubko, R., \& Cronin, A. D. (2015). Measurements of the ground-state polarizabilities of cs, rb, and $\mathrm{k}$ using atom interferometry. Phys. Rev. A, 92, 052513.

[13] Han, J., Jamil, Y., Norum, D. V. L., Tanner, P. J., \& Gallagher, T. F. (2006). $\mathrm{Rb} n f$ quantum defects from millimeter-wave spectroscopy of cold ${ }^{85} \mathrm{Rb}$ rydberg atoms. Phys. Rev. A, 74, 054502.

[14] Holmgren, W. F., Revelle, M. C., Lonij, V. P. A., \& Cronin, A. D. (2010). Absolute and ratio measurements of the polarizability of na, $\mathrm{k}$, and $\mathrm{rb}$ with an atom interferometer. Phys. Rev. A, 81, 053607.

[15] Hughes, H. K. (1947). The electric resonance method of radiofrequency spectroscopy the moment of inertia and electric dipole moment of csf. Phys. Rev., 72, $614-623$.

[16] Johansson, I. (1961). Ark. Fys., 20, 135-146.

[17] Johnson, L. A. M. (2011). Precision Laser Spectroscopy of Rubidium with a Frequency Comb. PhD thesis, The University of Leeds.

[18] Johnson, W., Kolb, D., \& Huang, K.-N. (1983). Electric-dipole, quadrupole, and magnetic-dipole susceptibilities and shielding factors for closed-shell ions of the he, ne, ar, ni (cu+), kr, pb, and xe isoelectronic sequences. Atomic Data and Nuclear Data Tables, 28(2), 333 - 340.

[19] Kim, E. G., Nunkaew, J., \& Gallagher, T. F. (2014). Detection of barium $6 s n g \rightarrow 6 s n h, 6 s n i$, and $6 s n k$ microwave transitions using selective excitation to autoionizing states. Phys. Rev. A, 89, 062503.

[20] Li, W., Mourachko, I., Noel, M. W., \& Gallagher, T. F. (2003). Millimeter-wave spectroscopy of cold rb rydberg atoms in a magneto-optical trap: Quantum defects of the ns, np, and nd series. Phys. Rev. A, 67, 052502.

[21] Lim, I. S., Laerdahl, J. K., \& Schwerdtfeger, P. (2002). Fully relativistic coupledcluster static dipole polarizabilities of the positively charged alkali ions from li+ to 119+. The Journal of Chemical Physics, 116(1), 172-178.

[22] Mayer, J. E. \& Mayer, M. G. (1933). The polarizabilities of ions from spectra. Phys. Rev., 43, 605-611. 
[23] Miffre, A., Jacquey, M., Büchner, M., Trénec, G., \& Vigué, J. (2006). Measurement of the electric polarizability of lithium by atom interferometry. Phys. Rev. A, 73, 011603 .

[24] Mitroy, J., Safronova, M. S., \& Clark, C. W. (2010). Theory and applications of atomic and ionic polarizabilities. Journal of Physics B: Atomic, Molecular and Optical Physics, 43(20), 202001.

[25] Nipper, J., Balewski, J. B., Krupp, A. T., Butscher, B., Löw, R., \& Pfau, T. (2012). Highly resolved measurements of stark-tuned förster resonances between rydberg atoms. Phys. Rev. Lett., 108, 113001.

[26] Nunkaew, J. \& Gallagher, T. F. (2015). Microwave spectroscopy of the calcium $4 s n f \rightarrow 4 s(n+1) d, 4 s n g, 4 s n h, 4 s n i$, and $4 s n k$ transitions. Phys. Rev. A, 91, 042503 .

[27] Nunkaew, J., Shuman, E. S., \& Gallagher, T. F. (2009). Indirect spin-orbit $k$ splittings in strontium. Phys. Rev. A, 79, 054501.

[28] Park, H., Tanner, P. J., Claessens, B. J., Shuman, E. S., \& Gallagher, T. F. (2011). Dipole-dipole broadening of $\mathrm{rb} n s-n p$ microwave transitions. Phys. Rev. A, 84, 022704 .

[29] Reinhard, A., Cubel Liebisch, T., Younge, K. C., Berman, P. R., \& Raithel, G. (2008). Rydberg-rydberg collisions: Resonant enhancement of state mixing and penning ionization. Phys. Rev. Lett., 100, 123007.

[30] Safinya, K. A., Gallagher, T. F., \& Sandner, W. (1980). Resonance measurements of $f-h$ and $f-i$ intervals in cesium using selective and delayed field ionization. Phys. Rev. A, 22, 2672-2678.

[31] Safronova, M. S. \& Safronova, U. I. (2011). Critically evaluated theoretical energies, lifetimes, hyperfine constants, and multipole polarizabilities in ${ }^{87} \mathrm{Rb}$. Phys. Rev. A, 83, 052508.

[32] Sternheimer, R. M. (1970). Quadrupole polarizabilities of various ions and the alkali atoms. Phys. Rev. A, 1, 321-327.

[33] Sternheimer, R. M., Rodgers, J. E., \& Das, T. P. (1978). Effect of the atomic core on the fine-structure splitting for excited nd and nf states of the alkali-metal atoms. Phys. Rev. A, 17, 505-512. 
[34] Stevens, G. D. \& Lundeen, S. R. (1999). Measurements of g-h and h-i finestructure intervals in $n=7,9$, and 10 helium rydberg states. Phys. Rev. A, 60, 4379-4386.

[35] Tran, N. H., van Linden van den Heuvell, H. B., Kachru, R., \& Gallagher, T. F. (1984). Radiofrequency resonance measurements of na $d-f$ intervals. Phys. Rev. A, 30, 2097-2099. 


\section{Chapter 6}

\section{Future Work}

\subsection{Introduction}

This chapter describes some interesting experimental observations that were made throughout the course of my studies that I would like to pursue further in the future.

\section{$6.2 n s_{1 / 2} n s_{1 / 2} \rightarrow n p_{1 / 2} n p_{3 / 2}$ Transition}

Figs. 6.1 and 6.2 show the 39p signal at high and low microwave power (with density kept at maximum value) and at high and low density (with microwave power kept at maximum value), respectively, as the microwave frequency is swept across the resonance frequencies of the two atomic transitions $39 s_{1 / 2} \rightarrow 39 p_{1 / 2}$ and $39 s_{1 / 2} \rightarrow$ $39 p_{3 / 2}$.

Fig. 6.1 shows that at low microwave power the atomic $39 s_{1 / 2} \rightarrow 39 p_{1 / 2}$ and $39 s_{1 / 2} \rightarrow 39 p_{3 / 2}$ transitions occur at the calculated frequencies of 66.5696 and 68.3787 


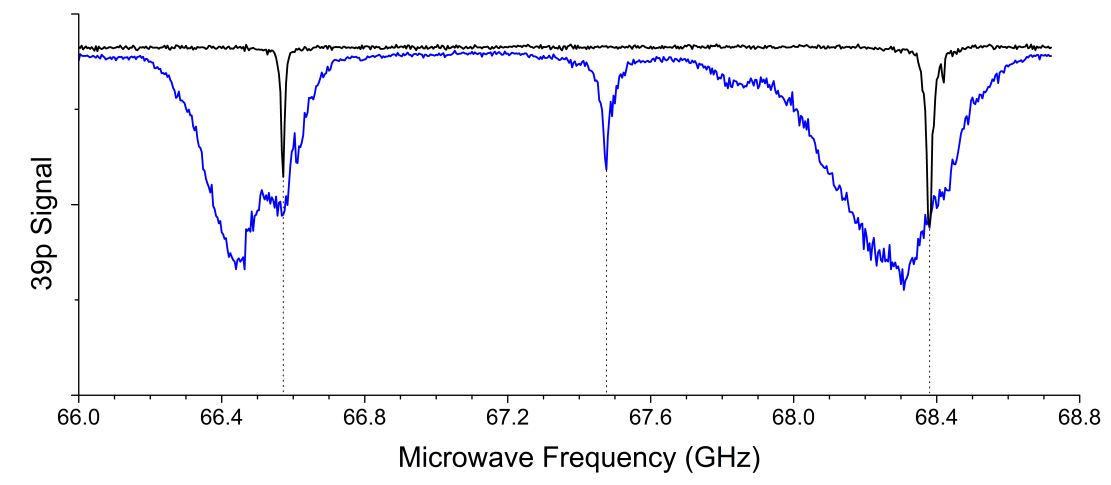

Figure 6.1: The figure shows the 39p signal at high (blue trace) and low (black trace) microwave power as the microwave frequency is swept across the resonance frequencies of the two atomic transitions $39 s_{1 / 2} \rightarrow 39 p_{1 / 2}$ and $39 s_{1 / 2} \rightarrow 39 p_{3 / 2}$. The Rydberg atom density is kept the same. At high microwave power, there is a new peak at $67.474 \mathrm{GHz}$.

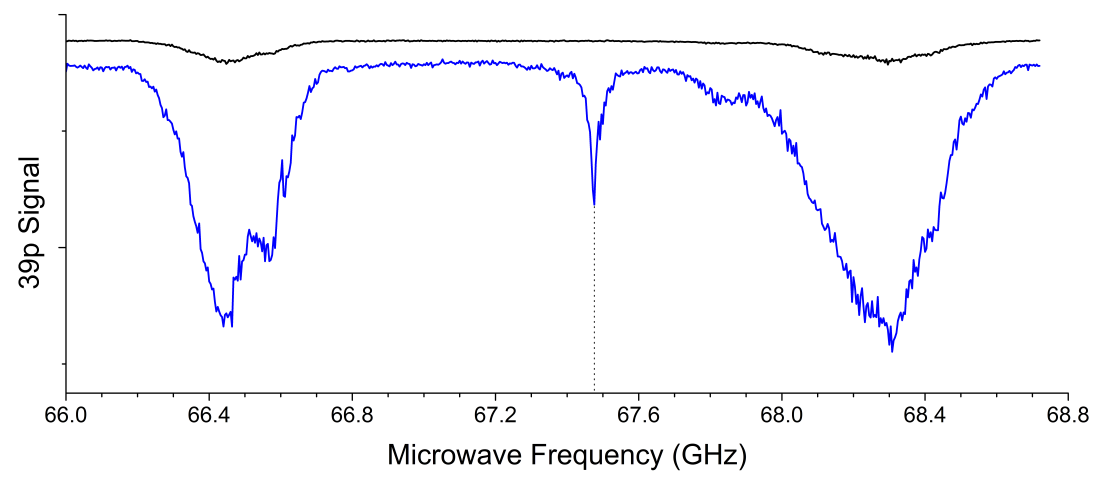

Figure 6.2: The figure shows the 39p signal at high (blue trace) and low (black trace) Rydberg atom densities as the microwave frequency is swept across the resonance frequencies of the two atomic transitions $39 s_{1 / 2} \rightarrow 39 p_{1 / 2}$ and $39 s_{1 / 2} \rightarrow 39 p_{3 / 2}$. The microwave power is kept the same. At high density, there is a new peak at 67.474 $\mathrm{GHz}$. 


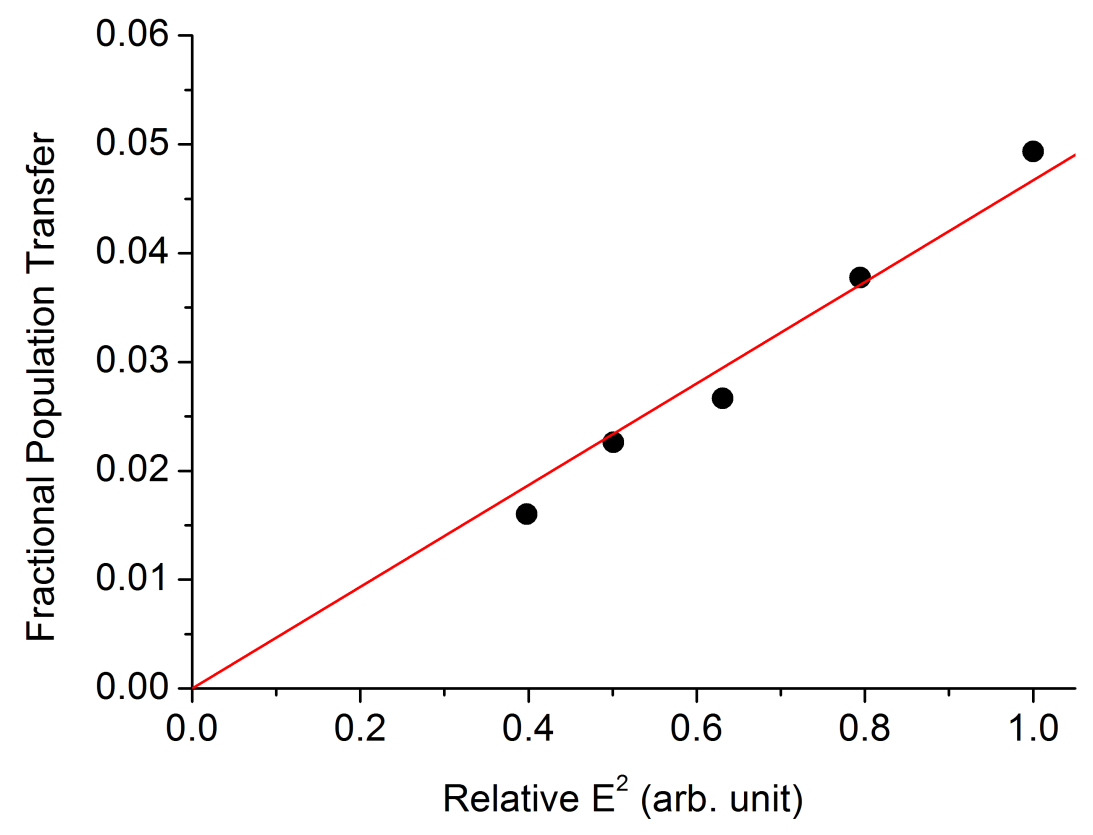

Figure 6.3: $\quad$ FPT vs. power for the $39 s_{1 / 2} 39 s_{1 / 2} \rightarrow 39 p_{1 / 2} 39 p_{3 / 2}$ transition.

$\mathrm{GHz}$ as expected. When the microwave power is increased, the Rabi frequencies of the atomic resonances are increased to about $400 \mathrm{MHz}$. The resonant peak that corresponds to $39 s_{1 / 2} \rightarrow 39 p_{3 / 2}$ is broader because the oscillator strength for $39 s_{1 / 2} \rightarrow 39 p_{3 / 2}$ is about 2 times greater than for $39 s_{1 / 2} \rightarrow 39 p_{1 / 2}$. At the high microwave power, however, there is an additional peak at the frequency that is exactly halfway between $39 s_{1 / 2} \rightarrow 39 p_{1 / 2}$ and $39 s_{1 / 2} \rightarrow 39 p_{3 / 2}$ transition frequencies. The frequency matches the transition frequency for the transition between the pair states $39 s_{1 / 2} 39 s_{1 / 2}$ and $39 p_{1 / 2} 39 p_{3 / 2}$. Fig. 6.2 shows that the additional peak disappears when the Rydberg atom density is reduced by attenuating the excitation laser intensity. 


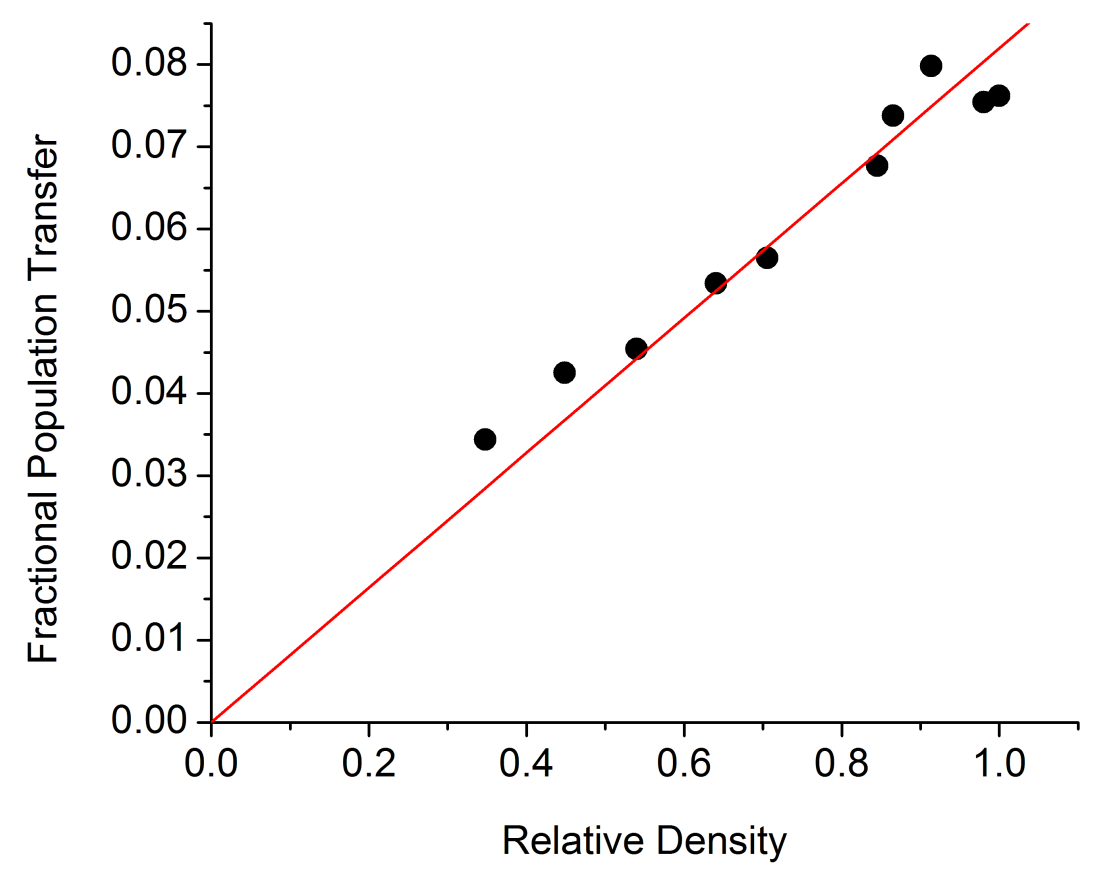

Figure 6.4: FPT vs. density for the $39 s_{1 / 2} 39 s_{1 / 2} \rightarrow 39 p_{1 / 2} 39 p_{3 / 2}$ transition. 
To investigate this transition further, systematic measurements of the fractional population transfer (FPT) when the microwave frequency is tuned to the $39 s_{1 / 2} 39 s_{1 / 2} \rightarrow$ $39 p_{1 / 2} 39 p_{3 / 2}$ resonance at different microwave powers and Rydberg atom densities were made. Figs. 6.3 and 6.4 show plots of FPT vs. microwave power and FPT vs. density.

In both cases, the FPT scales linearly. Since the microwave power is proportional to the square of the field amplitude, the fact that FPT scales linearly with the microwave power means that FPT is proportional to the square of the microwave field amplitude. This suggests that the transition is likely a two photon transition, for which the FPT depends on the square of the field amplitude. The fact that FPT scales linearly with the density suggests that the transition probability is proportional to the number of atoms squared, which implies that this is likely a process that involves two atoms rather than one.

The resonant peak does not shift or broaden when the microwave power is raised. The resonant peak does not shift with the density either, but the linewidth decreases slightly when density is reduced. When the relative density is changed from 1 to 0.15, the linewidth changes from 24 to $11 \mathrm{MHz}$.

One aspect of this transition that is difficult to understand is the asymmetric shape of the resonant peak. Fig. 6.5 shows the resonant peaks at different densities. The "wing" on the lower frequency side is clearly visible.

Several features of the obtained data led us to believe that this is the $39 s_{1 / 2} 39 s_{1 / 2} \rightarrow$ $39 p_{1 / 2} 39 p_{3 / 2}$ transition between pair states. First, the frequency of the observed resonance peak matches the $39 s_{1 / 2} 39 s_{1 / 2} \rightarrow 39 p_{1 / 2} 39 p_{3 / 2}$ transition to within $1 \mathrm{MHz}$. It 
had also been checked that there are no other possible atomic or molecular transitions at or near the frequency of the observed transition. Second, the linear scaling of the FPT with the density suggests that this process involves two atoms. Third, the linear scaling of the FPT with the microwave field amplitude squared combined with the relatively high microwave field amplitude required to observe the transition (the minimum microwave field amplitude required to be able to observe the transition is $75 \mathrm{mV} / \mathrm{cm}$ ) suggests that this is a two photon process.

With those being said, we were unable to determine what mechanism was allowing this process to occur. Many simulation attempts using the 2-atom Floquet model and/or 2-atom configuration interaction model did not produce sensible results. However, until now we have been making a few assumptions. First, we assumed that two dipoles are aligned along the z-axis. Second, we assumed that the microwaves only have linear polarization. Both may be oversimplifications - because the two dipoles are not necessarily aligned along the z-axis and the rods used to apply field ionization pulse scatters microwaves which could results in the polarization's not being perfectly linear. Future simulations without making these assumptions may provide insights on how this transition occurs. Finding out how the transition occurs would be a very interesting project that builds upon the previous experiments on the microwave transition between pair states of Rydberg atoms. 


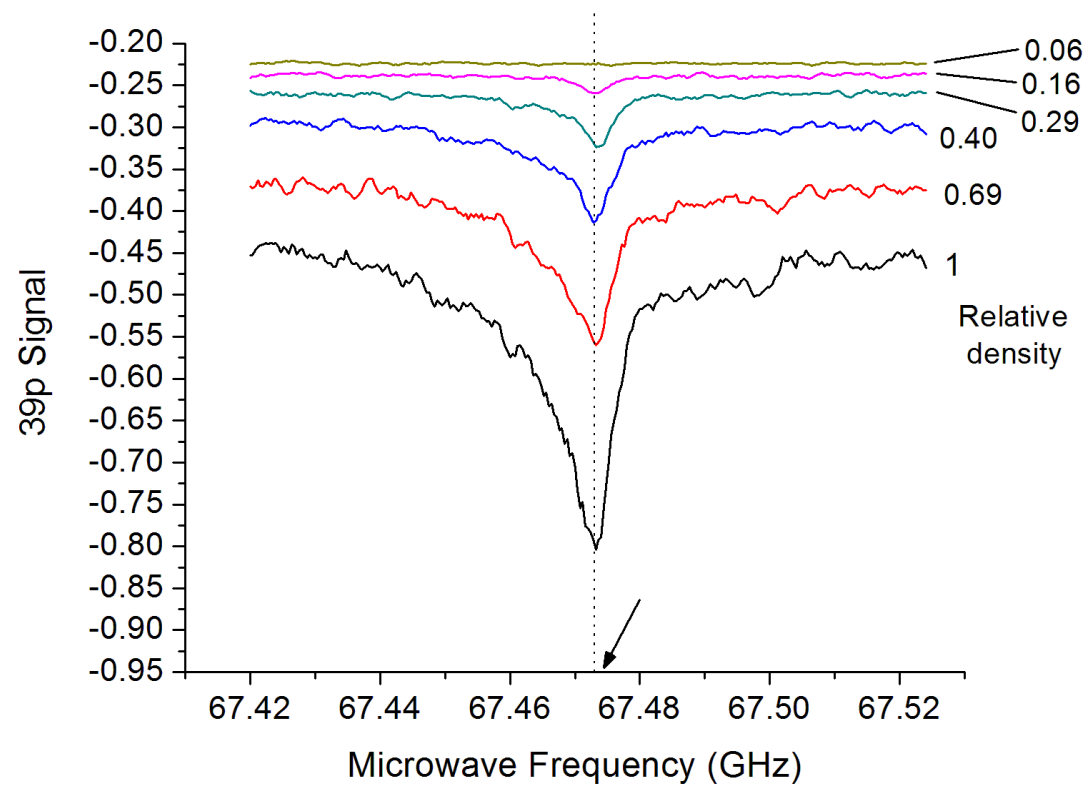

Figure 6.5: The resonant peaks at different densities. The "wing" on the lower frequency side is clearly visible. 


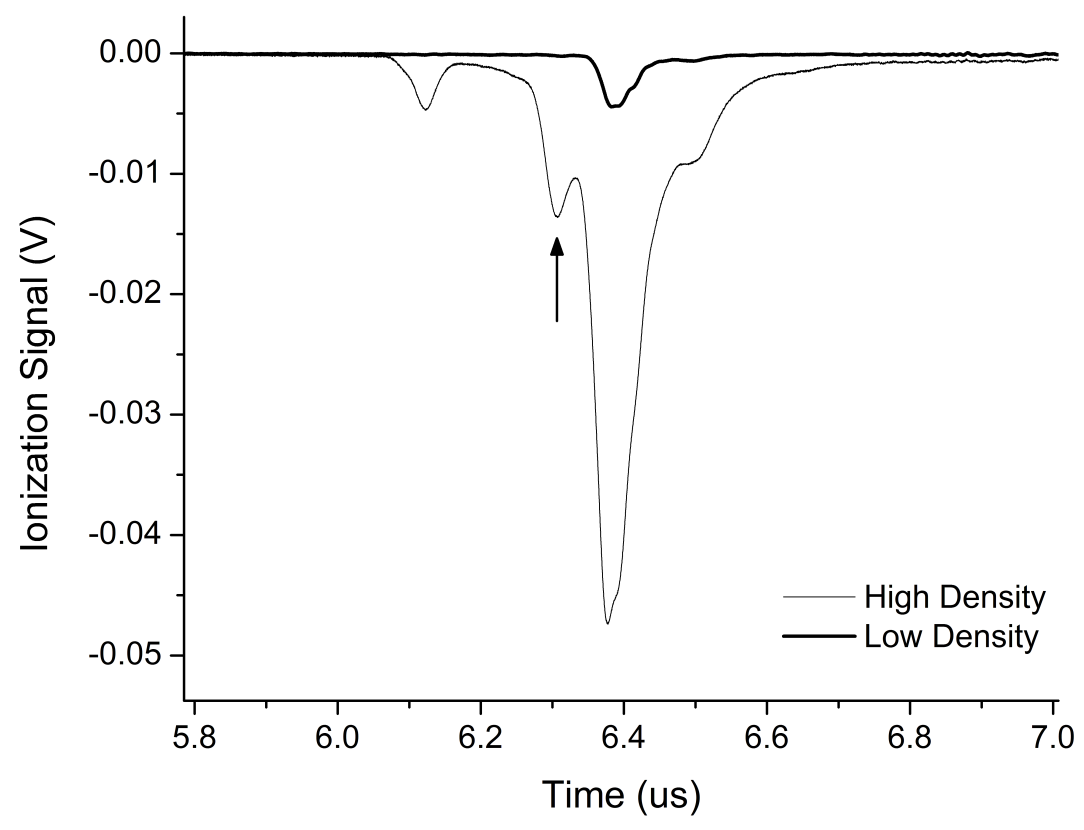

Figure 6.6: Time resolved field ionization signals from a sample of atoms excited to the $42 d$ state at high and low density. Ions are being detected. 


\subsection{Formation of $(n+2) p$ atoms}

Fig. 6.6 shows oscilloscope traces of the signal from the MCP detector when the trapped atoms in the MOT are excited to the $42 d_{5 / 2}$ state. At low density, there is only one peak. However, at high density there are additional peaks that come earlier in time at $6.1 \mu \mathrm{s}$ and $6.3 \mu \mathrm{s}$. The timing of the peak at $6.1 \mu \mathrm{s}$ corresponds to either free ions or Rydberg atoms in higher lying states. The timing of the peak at 6.3 $\mu$ s corresponds to either $(n+2) p$ or $(n+1) d$ states. Although the origin of both peaks are unknown, only the peak at $6.3 \mu$ s (shown by the arrow) will be discussed here. This phenomenon of additional features appearing in field ionization signal is not only restricted to the case of $42 d_{5 / 2}$. This has been also observed with a sample excited to $44 d_{5 / 2}, 41 d_{5 / 2}, 40 d_{5 / 2}$, and $39 d_{5 / 2}$ states.

For convenience, we shall refer to this signal as the "apparent" $(n+2) p$ signal. The ratio of the "apparent" $(n+2) p$ signal to the time integrated signal was measured as either the density of Rydberg atoms or the time delay of the field ionization pulse was changed. They are shown in Figs. 6.7 and 6.8. It can be seen that the ratio increases linearly in both cases. The fact that the ratio increases linearly with density means that the size of the "apparent" $(n+2) p$ signal scales quadratically with density.

Han[1] suggested that the field ionization pulse with slow initial rise can lead to an appearance of $(n+2) p$ signal by allowing adiabatic passage through the molecular avoided crossings between $n d n d$ and $(n+2) p(n-2) f$ states. While this seems reasonable, especially given the fact that the y-intercept of Fig. 6.8 is not zero, we suspect that there is another mechanism at work that is producing free $(n+2) p$ atoms even before the field ionization pulse comes in. 


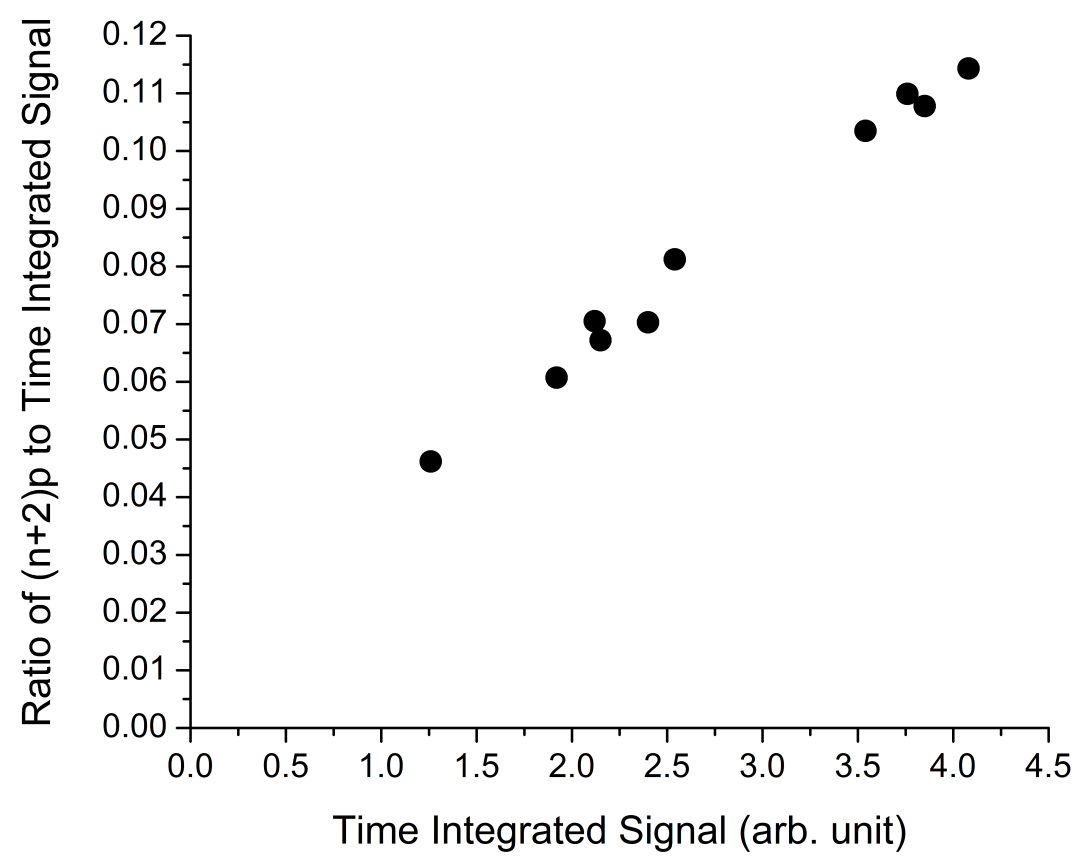

Figure 6.7: The ratio of the "apparent" $(n+2) p$ signal vs. time integrated signal as the density of Rydberg atoms is changed. 


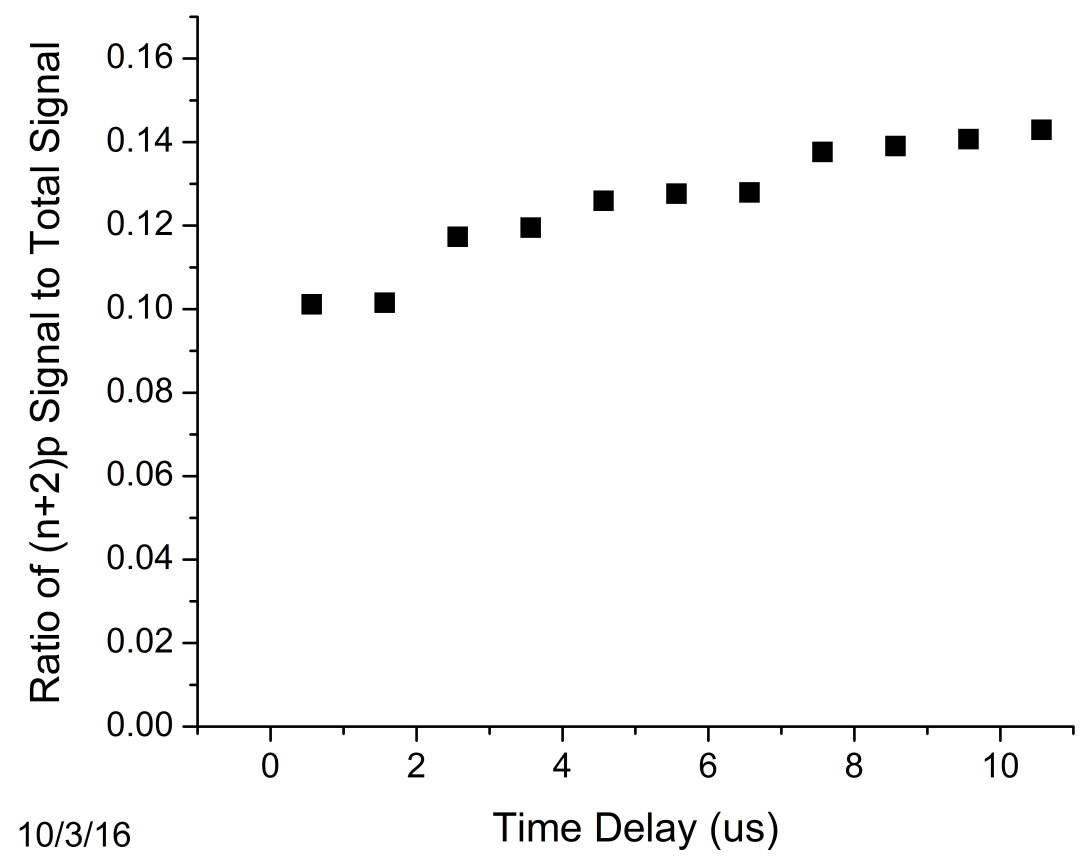

Figure 6.8: The ratio of the "apparent" $(n+2) p$ signal vs. time integrated signal as the delay of the field ionization pulse is changed. 
The claim that there are free $(n+2) p$ atoms before the field ionization is based on an observation that it is possible to drive the two photon atomic transition $(n+2) p \rightarrow$ $(n+3) p$ and detect the signal from the resulting $(n+3) p$ atoms. Fig. 6.9 shows the field ionization signal from the $42 d$ state with the microwaves turned on and off. The microwave frequency is tuned to the transition frequency of the two photon transition $44 p_{3 / 2} \rightarrow 45 p_{3 / 2}$. It is clear that the $44 p$ signal decreases and the $45 p$ signal increases when the microwaves are turned on. In addition, Fig. 6.10 shows the resonant peak for the $44 p_{3 / 2} \rightarrow 45 p_{3 / 2}$ transition that was observed by placing the gate of a boxcar integrator on the $45 p_{3 / 2}$ signal while sweeping the microwave frequency. The observed transition frequency of $44.870 \mathrm{GHz}$ shows an excellent agreement with the calculated frequency of $44.870 \mathrm{GHz}$. 


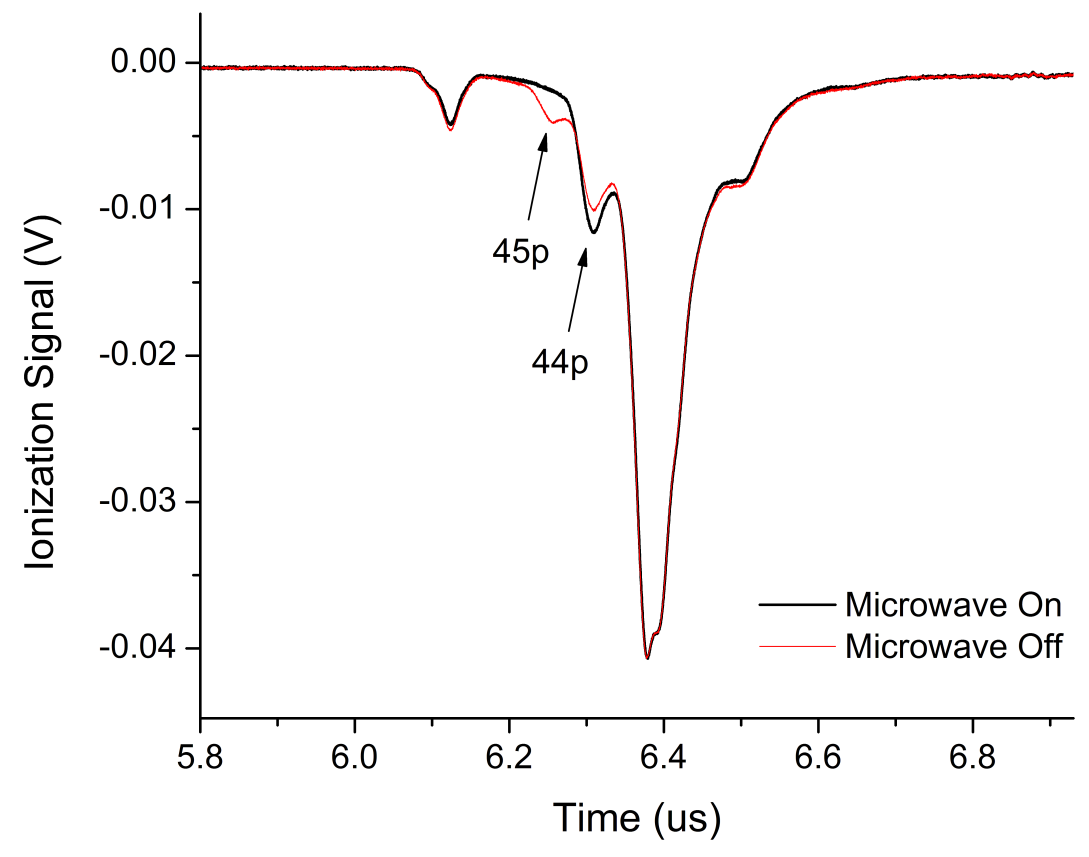

Figure 6.9: The ionization signal from a sample of atoms excited to $42 d$ state with microwave turned on and off. Microwave frequency is tuned to the transition frequency of the two photon transition $44 p_{3 / 2} \rightarrow 45 p_{3 / 2}$. 


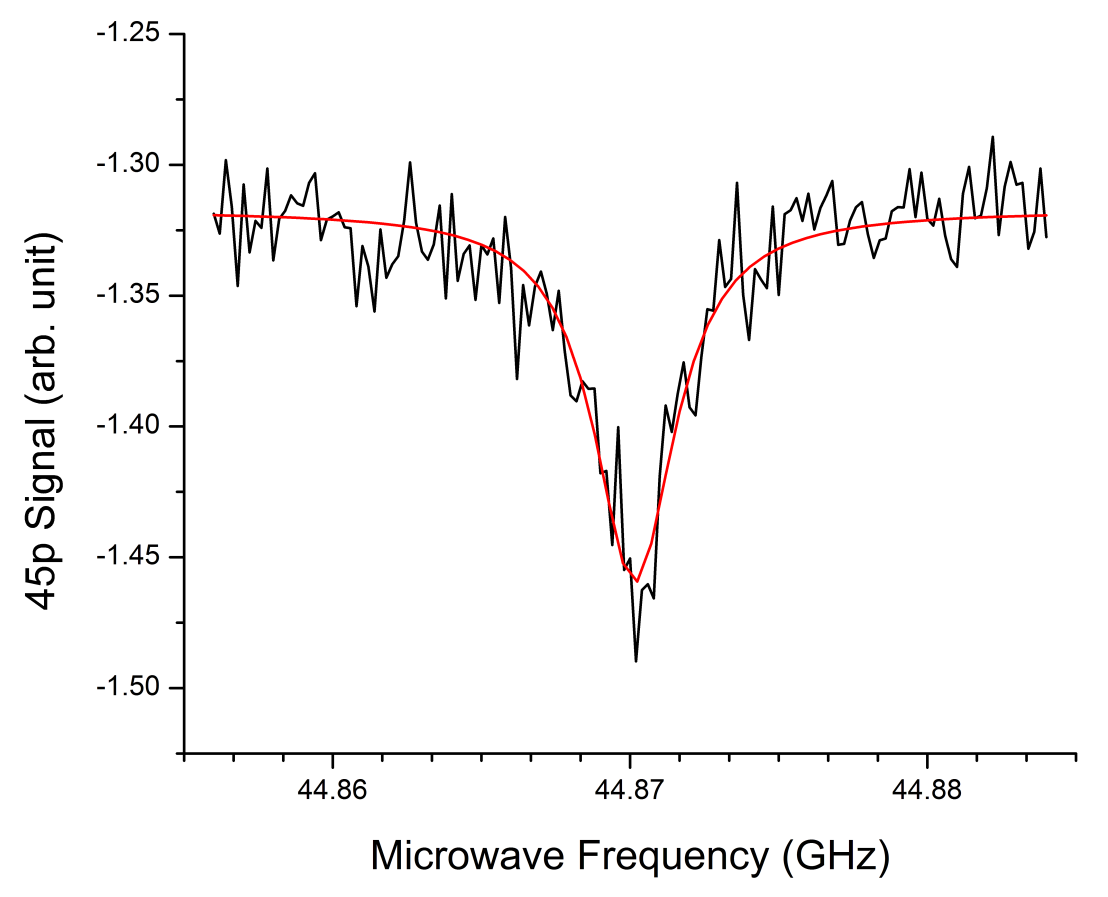

Figure 6.10: The resonant peak for the $44 p_{3 / 2} \rightarrow 45 p_{3 / 2}$ transition. The observed transition frequency, from the Lorentzian fit, is $44.872 \mathrm{GHz}$ which shows a good agreement with the calculated frequency of $44.870 \mathrm{GHz}$. 
While the observation of this transition suggests that free $(n+2) p$ atoms are being produced shortly after the excitation to $n d$ Rydberg state, the mechanism is not yet understood. It was suspected that the presence of hot, room-temperature atoms in the MOT and their colliding with the Rydberg atoms may be responsible for the formation of $(n+2) p$. However, an attempt to purposely increase the number of "hot" atoms into the MOT did not noticeably affect the number of $(n+2) p$ atoms produced.

There are a few additional experiments that can be done to gain more insight. The first is to make a systematic measurements of how $(n+2) p_{3 / 2} \rightarrow(n+3) p_{3 / 2}$ resonant peak changes as the density of Rydberg atoms is increased. The second is to modify the field ionization pulse profile slightly (by adding some noise or spikes) to observe what effects it has on the "apparent" $(n+2) p$ signal. 


\section{REFERENCES}

[1] Han, J. \& Gallagher, T. F. (2008). Field ionization of the frozen rydberg gas. Phys. Rev. A, 77, 015404. 


\section{Chapter 7}

\section{Conclusion}

The unique condition created as a result of combining the exaggerated properties of Rydberg atoms and cold, dense environment provided by the magneto optical trap leads to observation of new phenomena. The microwave transitions between pair states, which was extensively studied in Chapter 3 and Chapter 4 of this dissertation, is one such example. This transition could only be observed because the dense sample of Rydberg atoms in a magneto optical trap led to a strong dipole-dipole interaction between $n d_{5 / 2} n d_{5 / 2}$ and nearby $(n+2) p_{3 / 2}(n-2) f$ states. It was shown that the configuration interaction (CI) model can be used to describe the single photon case quite well and give predictions that are consistent with the experimental results. In more general case with multi-photon transitions, Floquet-Forster picture provides convenient description of the transitions.

The values of quantum defects obtained from the experiment described in Chapter 5 were extracted from the systematic measurements of the zero field $(n+1) d \rightarrow n g$ 
and $(n+1) d \rightarrow n h$ intervals. A new experimental technique, which allowed us to obtain the zero field interval despite the fact that the stray field can only be controlled in one direction, was adopted to get the intervals. From the quantum defects, the ionic dipole and quadrupole polarizabilities were determined.

The observations in Section 6.2 suggests that there may be transitions between pair states that occur due to different mechanism, as the configuration interaction model does not provide a satisfactory explanation for the observed $39 s_{1 / 2} 39 s_{1 / 2} \rightarrow$ $39 p_{1 / 2} 39 p_{3 / 2}$ transition. The observations in Section 6.3 suggests that there may be a process, similar to spontaneous evolution of cold Rydberg atoms to plasma, but occurring in a shorter time scale that result in production of $(n+2) p$ atoms as well as higher lying states and/or free ions. In the future, it would be interesting to further investigate these phenomena. 